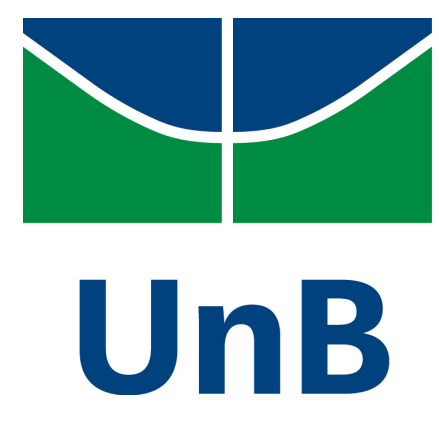

Universidade de Brasília

Instituto de Física

\title{
Uso do Einstein Toolkit na Gravidade Teleparalela
}

\author{
Dissertação de Mestrado \\ Hailleen Gisell Varela González
}

Orientador:

Dr. Sérgio Costa Ulhoa

Brasília - Brasil

Julho de 2016 


\section{"Uso do Einstein Toolkit na Gravidade Teleparalela."}

\section{Por}

\section{Hailleen Gisell Varela González.}

Dissertação submetida ao Instituto de Física da Universidade de Brasília como parte dos requisitos para a obtenção do grau de Mestre em Física.

Aprovada por:

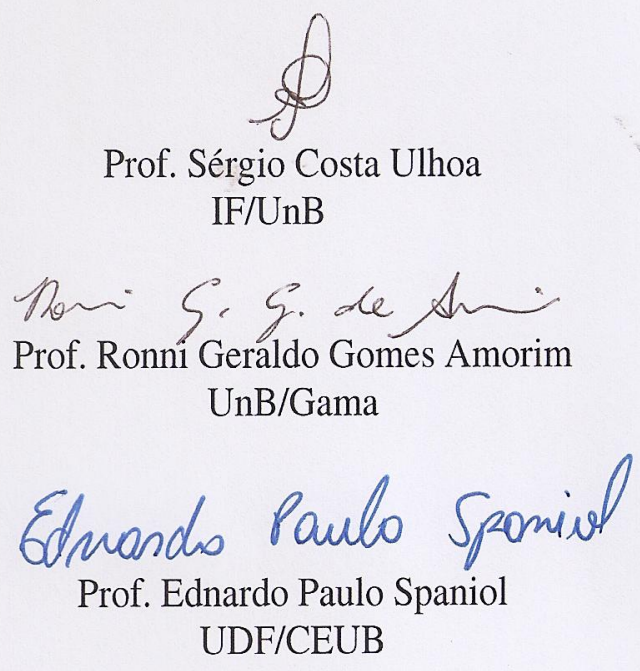

Prof. Dr. Fernando Albuquerque de Oliveira Coordenador de Pós-Graduação Instituto de Física 
"All of physics is either impossible or trivial. It is impossible until you understand it, and then it becomes trivial".

Ernest Rutherford 


\section{Agradecimentos}

Agradeço primeiramente a Deus, por todas as bênçãos que tenho recebido durante toda a minha vida e principalmente por ter me dado a maior presente de todos que é minha família. Agraceço a minha mãe, mi mamita linda, que apesar de não estar perto de mim sempre tem me apoiado em cada uma das minhas decisões. Agradeço também a meus irmãos Karen e Andres que sempre torceram por mim.

Agradeço ao Eduardo, meu companheiro de caminho, sem você não teria conseguido. Primeiramente por me dar a oportunidade de vir ao Brasil em busca de melhores condições de vida. Em segundo lugar, por sempre ter me apoiado nas minhas decisões e por ter tido muita paciência, carinho e amor. Agradeço também a teus pais, Jane Mara e Eduardo Pereira, pelo acolhimento na sua casa em um momento de dificuldade. Finalmente, quero agradecer ao Brasil por ter se mostrado uma pátria mãe gentil.

Quero agradecer ao meu orientador, o Dr. Sérgio Ulhoa Costa, que tão bem me acolheu em seu laboratório, por sua paciência, toda a atençã que sempre deu a mim e ao meu trabalho, por todas as oportunidades dadas e por contribuir tanto para a minha qualificação profissional. Agradeço também aos jurados Dr. Ronni Gomes Amorim e Dr. Ednardo Paulo Spaniol por sua contribuição para deixar este trabalho ainda melhor.

Agradeço a meus companheiros de sala de estudo, Rodrigo, Lydiane, Arthur, Carol, Neymar, Ana, por todas as dicas e ajuda no que se refere à vida acadêmica dentro da Universidade de Brasília. Agradeço ainda a outros companheiros do Instituto de Física, aos chamos David e Helena pela amizade e ótimos momentos compartilhados. Aqui deixar meu agradecimento especial a minha amiga Jucelia Silva quem me apresentou aquele que seria meu futuro orientador e sobretudo pela sua amizade e apoio durante meus estudos. Agradeço também ao Alexandre quem nunca deixou de atender a um pedido de ajuda, desde o mais simples ao mais complexo. Essa dedicação demonstra grande amizade e apreço, a você muito obrigada. Não poderia esquecer de todos aqueles amigos especiais que a vida me brindou e que desafortunadamente não podem estar fisicamente presentes mas que sua amizade se faz sentir de longe. Entre esses amigos eu gostaria de nomear a Douglas, Angelita, Walter, Gonzalo, Dany, Patty, Cristo, Francisco, Mariana, Carmona, Ratita, e muitos outros que de momento pode ser que tenham ficado de fora mas que moram no meu coração. A todos vocês muito obrigada. Por ultimo, mas não menos importante, agradeço a Diego, Grazielle, Felipe, Julia, Thiago, Isa, Dona Rita, por sua amizade e carinho.

Agradeço ao Cnpq pelo apoio financeiro que tornou possível minha estadia no Brasil. 


\section{Resumo}

O Teleparalelismo Equivalente à Relatividade Geral, mostra-se como uma alternativa à formulação métrica da Relatividade Geral, principalmente por permitir escrever, através de seu formalismo Lagrangiano, expressões consistentes para a energia e o momento angular gravitacionais. No presente trabalho foi calculada a densidade de energia gravitacional de uma estrela de nêutrons fazendo uso do formalismo teleparalelo em conjunto com a ferramenta numérico-computacional conhecida como Einstein Toolkit. Esta ferramenta fornece uma rotina já pré-definida onde são utilizadas as equações TOV para simular as características das estrelas de nêutrons, e que adicionalmente implementa o método evolutivo 3+1 BSSN.

Finalmente, se mostra a variação da densidade de energia ao longo dos eixos, evidenciando sua influência na dinâmica da estrela. 


\begin{abstract}
The teleparallel equivalent of general relativity shows itself as an alternative to the metric formulation of general relativity, particularly for allowing through its Lagrangian formalism, to write consistent expressions for gravitational energy and angular momentum. In this work it was calculated the gravitational energy density of a neutron star making use of the teleparallel formalism within the numeric computational infrastructure known as Einstein Toolkit. This tool provides a preset routine in which the TOV equations are used to simulate the neutron star features in addition to the implementation of the 3+1 BSSN evolution system. Finally it was showed the energy density variation along the axes showing its influence on the dynamics of the star.
\end{abstract}




\section{Sumário}

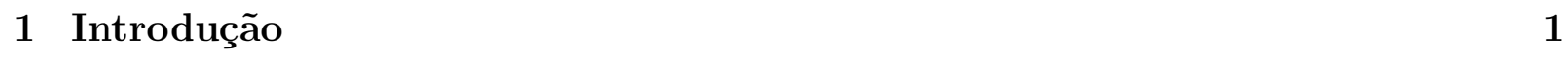

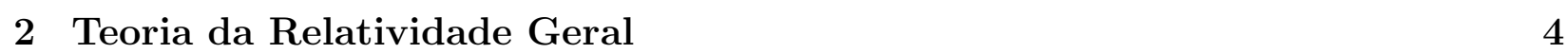

2.1 Teoria da Relatividade Especial . . . . . . . . . . . . . . . . . . . . . . . 4

2.2 Geometria Riemanniana $\ldots \ldots \ldots$. . . . . . . . . . . . . . . 9

2.3 Equações de Einstein pelo Princípio Variacional . . . . . . . . . . . . . . . . 15

2.4 Testes clássicos da Relatividade Geral . . . . . . . . . . . . . . . . . . . . . . 17

2.4 .1 O desvio anômalo do periélio de Mercúrio . . . . . . . . . . . . . . . 18

2.4 .2 A deflexão dos raios de luz de estrelas que passam na vizinhança solar 18

$2.4 .3 \quad$ O desvio para o vermelho da luz de origem gravitacional . . . . . . . 19

3 Teleparalelismo Equivalente à Relatividade Geral 21

3.1 Propriedades básicas do Campo de Tétradas . . . . . . . . . . . . . . . . . . 22

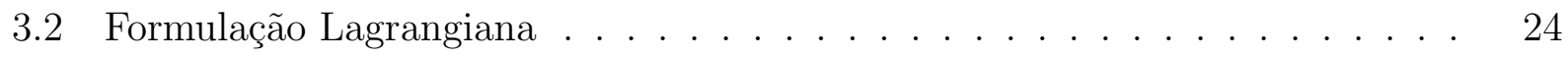

3.3 Interpretação do Campo de Tétradas . . . . . . . . . . . . . . . . . . . . . 34

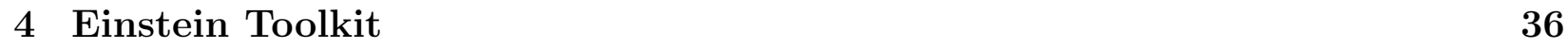

4.1 Visão Estrutural $\ldots \ldots$. . . . . . . . . . . . . . . . . . . . . . . 37

4.1 .1 Cactus . . . . . . . . . . . . . . . . . . . 37

4.1 .2 Carpet $\ldots \ldots \ldots \ldots \ldots$

4.1 .3 SimFactory $\ldots \ldots \ldots$

4.1 .4 GetComponents . . . . . . . . . . . . . . . . . . . . . 39

4.1 .5 Anatomia de um Thorn . . . . . . . . . . . . . . . . . . 40

4.2 Principais thorns . . . . . . . . . . . . . . . . . . . . 41

4.2 .1 EinsteinBase . . . . . . . . . . . . . . . . . . 41 
4.2 .2 EinsteinInitial . . . . . . . . . . . . . . . . . . . . . . . . . . . . 45

$4.2 .3 \quad$ EinsteinEvolve/McLachlan . . . . . . . . . . . . . . . . . . . . . 46

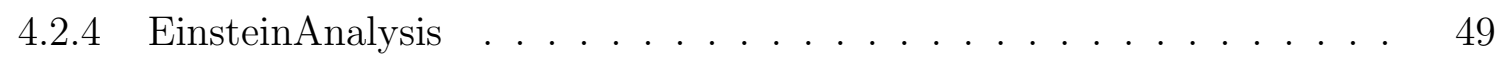

4.3 Implementação do Einstein Toolkit . . . . . . . . . . . . . . . . . . . . . . . 50

4.3 .1 Requerimentos . . . . . . . . . . . . . . . . 50

4.3 .2 Execução do ET . . . . . . . . . . . . . . . . . . . . . . 51

$\begin{array}{lll}5 & \text { Resultados e discussão } & 56\end{array}$

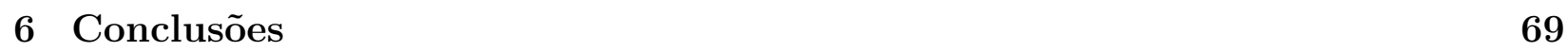




\section{Lista de Figuras}

2.1 Cone de luz de um evento $A$ e as diferentes regiões do espaço-tempo deste evento $7 \mid$. . . . . . . . . . . . . . . . . . . . . . . . . 8

4.1 Visão geral dos thorns centrais do arrangement EinsteinBase: ADMBase, HydroBase e TmunuBase em conjuto com alguns thorns escolhidos pelo usuário . . . . . 41

5.1 Representação de um pulsar, onde é mostrado o desalinhamento entre o eixo de rotação e os feixes de radiação emitida desde os pólos [54]. . . . . . . . . . 57

5.2 Vista parcial da tabela de dados gerados no arquivo ADMBase: :metric.x.asc produto da simulação da rotina static_tov.par. . . . . . . . . . . . . . . 64

$5.3 \quad$ Variação da densidade de energia gravitacional ao longo dos eixos $x, y$ e $z$. $\quad 67$

5.4 Evolução da densidade central de uma estrela TOV. . . . . . . . . . . . . . . 68 


\section{Capítulo 1}

\section{Introdução}

$\mathrm{Na}$ tentativa de formular uma teoria completamente covariante, capaz de descrever as leis da física, incluindo a gravidade, em qualquer sistema de coordenadas e para qualquer movimento relativo, Einstein estudou a similaridade entre referenciais inerciais e referenciais imersos em um campo gravitacional, concluindo assim que a gravidade e a aceleração são equivalentes. Usando o Princípio de Equivalência como base, Einstein apresentou em 1915 a extensão de sua teoria da Relatividade Especial a qual incluía os efeitos da gravidade e onde as leis da física são válidas em todos os referencias inerciais. Esta nova teoria é conhecida como a teoria da Relatividade Geral, onde trata-se a gravitação como uma consequência do espaço-tempo, enquanto que a curvatura do espaço-tempo é uma consequência da presença de matéria. No entanto, a curvatura do espaço-tempo afeta o movimento da matéria, a qual reciprocamente determina as propriedades geométricas e a evolução do espaço [1].

O Teleparalelismo equivalente à Relatividade Geral (TEGR), é uma formulação geométrica alternativa à teoria da Relatividade Geral de Einstein, onde os efeitos do campo gravitacional são descritos em termos dos campos de tétradas. As primeiras tentativas para descrever o campo gravitacional usando as tétradas são atribuídas a Einstein no seu esforço para unir a gravitação e o electromagnetismo [2]. Entretanto, usando esta aproximação ele não conseguiu encontrar uma descrição fiel e consistente das equações do campo eletromagnético. Esta nova teoria é totalmente equivalente à Relatividade, dado que ambas teorias são derivadas a partir de Lagrangianas, as quais diferem somente em uma divergência total, obtendo-se assim as mesmas equações de campo. Logo, a principal vantagem do Teleparalelismo sobre 
a Relatividade de Einstein está na possibilidade de escrever expressões consistentes para a energia, o momento e o momento angular [3].

A Relatividade Geral em conjunto com a mêcanica quântica, descreve o mundo como nós o conhecemos em seu nível mais fundamental. O problema é que há um conjunto muito pequeno de soluções para as equações de Einstein, além disso, estas soluções são todas para situações idealizadas. Uma forma de estudar situações mais realistas, como por exemplo um par de buracos negros orbitando-se mutuamente, se faz necessário resolver numericamente as equações de Einstein. Tradicionalmente, isto tem sido feito ou com cada pesquisador partindo do zero ou herdando um trabalho anterior de algum outro pesquisador. Mas agora existe um projeto que todos podem usar chamado Einstein Toolkit [4]. Este projeto começou a partir do código Cactus, o qual é um ambiente de trabalho que consiste de um núcleo central, chamado flesh, e um número de plugins conhecidos como thorns. O Cactus fornece um ambiente de trabalho genérico em computação científica para um grande número de campos. O Einstein Toolkit é uma ramificação do Cactus somente com os thorns necessários para a Relatividade Númerica.

Desde o ponto de vista dinâmico, tanto a Relatividade Geral de Einstein como a Gravidade Teleparalela fazem as mesmas predições. Embora o TERG permite a definição de quantidades de grande interesse físico, como a energia e momento angular gravitacionais, na Relatividade Geral ditas quantidades ainda estão em desenvolvimento. As estrelas de nêutrons são os laboratórios apropriados para o estudo da física de matéria densa, dado que elas contêm altas densidades de máteria fria do universo. Adicionalmente, as estrelas de nêutrons são ótimas candidatas para testar a teoria da relatividade Geral. O objetivo deste trabalho foi calcular a densidade de energia gravitacional de uma estrela de nêutrons através do formalismo teleparalelo em conjunto com a ferramenta numérico-computacional conhecida como Einstein Toolkit.

No capítulo 2, será apresentada uma revisão da teoria da Relatividade Geral, partindo desde a Relatividade Especial até chegar nos testes clássicos que comprovam dita teoria. A seguir, no capítulo 3 abordamos o formalismo do Teleparalelismo Equivalente à Relatividade 
Geral fazendo ênfase na formulação Lagrangiana. No capítulo 4 fazemos uma introdução ao Einstein Toolkit, seus principais componentes e se mostram alguns dos thorns mais utilizados para simulações astrofísicas. Os resultados do presente trabalho são apresentados no capítulo 5, enquanto que as conclusões encerram o trabalho.

Os índices de espaço-tempo $\mu, \nu, \ldots$ e os índices do grupo de Lorentz global $\mathrm{SO}(3,1)$ $a, b, \ldots$ variam de 0 a 3 . Logo, índices de espaço e tempo são indicados de acordo com $\mu=0, i, a=(0),(i)$. O campo e tétradas é denotado por $e^{a}{ }_{\mu}$, o tensor métrico do espaçotempo de Minlowski levanta e abaixa índices e é fixado por $\eta_{a b}=e_{a \mu} e_{b \nu} g^{\mu \nu}=(-,+,+,+)$. O determinante do campo de tétradas é indicado por $e=\operatorname{det}\left(e^{a}{ }_{\mu}\right)$. As unidades são fixadas com a escolha $G=c=1$, a menos que se diga o contrário. 


\section{Capítulo 2}

\section{Teoria da Relatividade Geral}

\subsection{Teoria da Relatividade Especial}

Motivado pela teoria electromagnética de Maxwell e pela ausência de evidência para a existência do éter, Albert Einstein formulou em 1905 a teoria da Relatividade Especial. A condição de validade das equações de Maxwell em qualquer referencial inercial levava à invariância da velocidade da luz, contradizendo-se assim à Relatividade Galileana.

Em sua teoria, Einstein enunciou dois postulados: o primeiro, conhecido como o Princípio da Relatividade, estabelece que as leis da física são iguais em qualquer referencial inercial, em outras palavras, não existe referencial inercial privilegiado. O segundo postulado afirma que a velocidade da luz é constante no vácuo e tem o mesmo valor $c$ em todos os referenciais inerciais [1].

O Princípio da Relatividade de Einstein, supõe que as expressões matemáticas que descrevem qualquer fenômeno físico devem possuir a mesma forma em todos os referenciais inerciais, exigindo assim um novo conjunto de transformações para as grandezas físicas, de um referencial inercial para outro. Portanto, Einstein foi forçado a reavaliar as ideias de espaço e tempo aceitas para a época, e através de uma serie de simples experimentos mentais ele demonstrou que as limitações da mecânica Newtoniana radicavam no conceito de simultaneidade dos eventos. Desta forma, Einstein reobteve as transforma@ões de Lorentz sob as quais tanto as equações de Maxwell como as equações que descrevem as leis da mecânica são covariantes. 
Para escrever a forma exata destas transformações, consideremos uma fonte de luz localizada no ponto $x=y=z=0$, de um sistema de coordenadas $\mathcal{O}$ livre de campos gravitacionais. No instante $t=0$, a fonte é acesa emitindo luz em todas as direções, gerando-se assim uma frente de ondas esféricas que se propagam com velocidade $c$ e que no tempo $t$ define a superfície esférica

$$
-c^{2} t^{2}+x^{2}+y^{2}+z^{2}=0
$$

Na equação (2.1), as coordenadas espaciais e temporal entram de uma maneira simétrica, portanto vamos adaptar nossas coordenadas a esta esfera de luz onde tomaremos o tempo como a coordenada zero, $x^{0}=c t$. Mais precisamente

$$
x^{\mu}=(c t, x, y, z), \quad x_{\mu}=(-c t, x, y, z), \quad \operatorname{com} \mu=0, \ldots, 3 .
$$

As coordenadas $x^{\mu}$ e $x_{\mu}$ estão relacionadas através da métrica pseudo-euclidiana $\eta_{\mu \beta}$ da forma

$$
x_{\mu}=\eta_{\mu \beta} x^{\beta}
$$

onde $\eta_{\mu \beta}$ é conhecida como métrica de Minkowski e é dada por

$$
\eta_{\mu \beta}=\left(\begin{array}{cccc}
-1 & 0 & 0 & 0 \\
0 & 1 & 0 & 0 \\
0 & 0 & 1 & 0 \\
0 & 0 & 0 & 1
\end{array}\right)
$$

Consideremos agora outro sistema de coordenadas $\mathcal{O}^{\prime}$, também livre da ação de campos gravitacionais e relacionado com o referencial $\mathcal{O}$ por uma transformação de Galileu

$$
\left(c t^{\prime}, x^{\prime}, y^{\prime}, z^{\prime}\right)=(c t, x-v t, y, z)
$$

Se a velocidade da luz também for $c$ neste referencial, uma esfera de luz no instante $t$ deveria descrever uma esfera com a forma

$$
(x-v t)^{2}+y^{2}+z^{2}-c^{2} t^{2}=0 .
$$


As equações 2.1 e 2.6 obviamente são diferentes, mas como a mesma esfera não pode estar em dois locais diferentes, concluímos que as transformações de Galileu não são compatíveis com o princípio de constância da luz. Então, vamos a requerer que as novas transformações deixem invariante à esfera de luz 2.1) de modo que a esfera seja a mesma nos dois referenciais. Como no caso da mecânica clássica, devemos ter transformações lineares, onde se satisfaça a condição [5]

$$
x^{\mu^{\prime}} x_{\mu^{\prime}}=\Lambda_{\alpha}^{\mu^{\prime}} \Lambda_{\mu^{\prime}}{ }^{\beta} x^{\alpha} x_{\beta}=x^{\beta} x_{\beta},
$$

onde, para todos os $x^{\alpha}$ somente é possível se

$$
\Lambda_{\alpha}^{\mu^{\prime}} \Lambda_{\mu^{\prime}}^{\beta}=\delta_{\alpha}^{\beta}, \quad \operatorname{com} \alpha, \beta, \mu^{\prime}=0, \ldots, 3
$$

Nas equações (2.7) e (2.8) introduzimos a conhecida notação de Einstein, onde índices covariantes e índices contravariantes iguais em qualquer termo, indicam soma sob ditos índices desde 0 até 3 .

Se consideramos agora que o referencial $\mathcal{O}^{\prime}$ se move ao longo do eixo $x$ com velocidade $v$ medida por $\mathcal{O}$, onde dizemos que este movimento é um boots na direção $x$ [6], as transformações de Lorentz que descrevem as transformações entre os dois sistemas são

$$
t^{\prime}=\frac{t-v x / c^{2}}{\sqrt{1-v^{2} / c^{2}}}, \quad x^{\prime}=\frac{x-v t}{\sqrt{1-v^{2} / c^{2}}}, \quad y^{\prime}=y, \quad z^{\prime}=z
$$

Do conjunto de equações (2.9) temos algumas consequências interessantes nas medições de tempo e comprimento. A primeira consequência, conhecida como Contração de Lorentz, está relacionada com o comprimento de um corpo o qual é medido para ser máximo quando se encontra em repouso com relação a um observador. Porém, quando o corpo se move com uma velocidade $v$, dito comprimento é diminuído na direção do movimento pelo fator $\sqrt{1-v^{2} / c^{2}}$, enquanto suas dimenções perpendiculares não são afetadas. Por outro lado, a segunda consequência trata de um relógio em movimento onde o tempo transcorre mais devagar do que em um relógio em repouso, ou ainda, em qualquer relógio o tempo corre à velocidade máxima desde o ponto de vista do observador em repouso. A este fenômeno dá-se o nome de Dilatação Temporal. Finalmente, temos como terceira consequência a inexistência 
de Simultaneidade. Dois eventos simultâneos, em um dado referencial inercial, acontecem no mesmo instante de tempo, no entanto, a transformação temporal de Lorentz sugere que em qualquer outro referencial inercial estes eventos podem não ser simultâneos. Em outras palavras, podemos dizer que dois relógios sincronizados em um dado referencial inercial podem não estar sincronizados em outros referencias inerciais.

As transformações de Lorentz mostram que o tempo deve ser tratado como uma coordenada ordinária, permitindo-nos imaginar a fusão do espaço e do tempo em uma única entidade conhecida como espaço-tempo. Este conceito foi incorporado na Relatividade Especial usando o espaço-tempo de Minkowski, ou simplesmente espaço de Minkowski. Para estudar as propriedades métricas do espaço de Minkowski consideremos dois eventos separados infinitesimalmente um de outro em um referencial $\mathcal{O}$, logo o intervalo entre eles é dado pela expressão

$$
d s^{2}=-c^{2} d t^{2}+d x^{2}+d y^{2}+d z^{2}=\eta_{\mu \beta} d x^{\mu} d x^{\beta}
$$

onde $\eta_{\mu \beta}$ está dada pela equação (2.4). Note-se que a quantidade $d s^{2}$ é invariante sob qualquer transformação de Lorentz do tipo $x^{\nu^{\prime}}=\Lambda^{\nu^{\prime}}{ }_{\alpha} x^{\alpha}$, dado que $x^{\nu} x_{\nu}$ é invariante. Por outro lado, como o elemento de linha $d s^{2}$ é uma propriedade somente dos dois eventos e não do observador, podemos fazer a seguinte classificação da relação entre os eventos. Se $d s^{2}$ é positivo dizemos que os eventos estão separados por uma linha tipo espaço. Se $d s^{2}$ é negativo, os eventos são separados por uma linha tipo tempo. Finalmente, se $d s^{2}=0$, ditos eventos se encontram separados tipo luz.

Eventos tipo luz separados de um evento particular $A$, formam um cone cujo ápice é o mesmo evento $A$, e que é conhecido como cone de luz. Por outro lado, todos os eventos que se localizam dentro do cone estão separados tipo tempo de $A$, assim como todos os eventos que se acham fora do cone estão separados tipo espaço. Logo, todos os eventos dentro do cone podem ser alcançados desde o evento $A$ por um objeto físico, enquanto os que estão fora nunca serão alcançados. Por esta razão, os eventos que se localizam dentro do "futuro" do cone são chamados de futuro absoluto do evento $A$, ao mesmo tempo que aqueles que se encontram no "passado" são conhecidos como passado absoluto, e aqueles que estão fora são chamados de outro lugar. Na figura (2.1) se ilustram as diferentes regiões do espaço-tempo. 


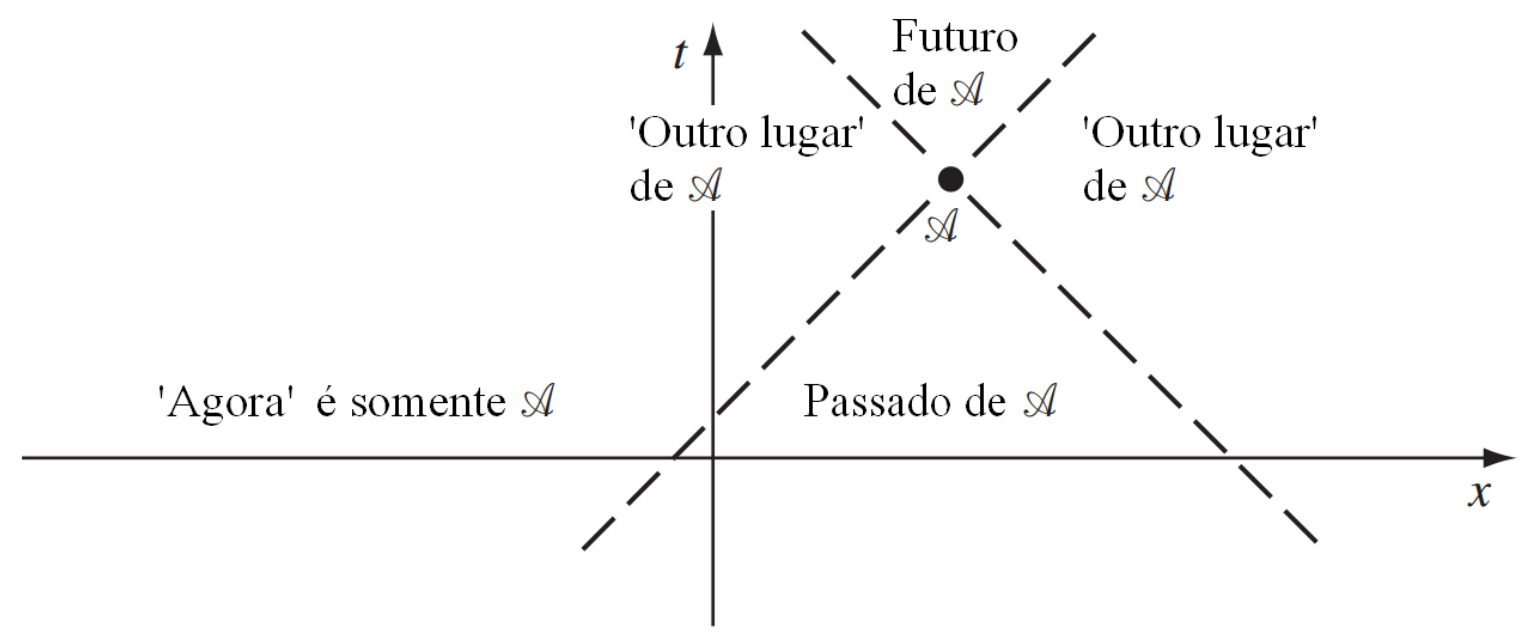

Figura 2.1: Cone de luz de um evento $A$ e as diferentes regiões do espaço-tempo deste evento 7 .

Na Relatividade Especial, as linhas mundo de duas partículas livres que começam paralelas uma da outra, permanecem nesse estado de movimento sem importar quão longe elas vão. Em outras palavras, o espaço de Minkowski é um espaço plano que obedece ao axioma do paralelismo de Euclides. No entanto, o espaço-tempo não é um espaço Euclidiano, dado que sua métrica é diferente. Se consideramos agora um referencial na presença de um campo gravitacional não uniforme, as linhas mundo de duas partículas geralmente não permanecem paralelas. Logo, o novo espaço-tempo gravitacional não é um espaço plano.

Na tentativa de formular uma teoria completamente covariante, capaz de descrever as leis da física, incluindo a gravidade, em qualquer sistema de coordenadas e para qualquer movimento relativo, Einstein estudou a similaridade entre referenciais inerciais acelerados e referenciais imersos em um campo gravitacional, concluindo assim que a gravidade e a aceleração são equivalentes. Partindo desta descoberta, Einstein formulou o conhecido Princípio da Equivalência entre gravidade e aceleração [7], o qual postula que qualquer experimento físico local que não implique gravidade vai ter o mesmo resultado se o executamos já seja 
em um sistema inercial em queda livre ou no espaço-tempo plano da Relatividade Especial. Como consequência direta do Príncipio de Equivalência temos que em um referencial inercial local, todas as leis da física conservam a forma da Relatividade especial, exceto a gravidade, a qual simplesmente desaparece.

Usando o Princípio de Equivalência como base, Einstein apresentou em 1915 a extensão de sua teoria da Relatividade Especial a qual incluía os efeitos da gravidade e onde as leis da física são válidas em todos os referencias inerciais. Esta nova teoria é conhecida como a teoria da Relatividade Geral, onde trata-se a gravitação como uma consequência do espaço-tempo, enquanto que a curvatura do espaço-tempo é uma consequência da presença de matéria. No entanto, a curvatura do espaço-tempo afeta o movimento da matéria, a qual reciprocamente determina as propriedades geométricas e a evolução do espaço.

Para descrever rigorosamente a curvatura do espaço-tempo precisamos de ferramentas matemáticas contidas na geometria diferencial, mais especificamente das Variedades Riemannianas, as quais são espaços-tempo localmente planos e onde o elemento de linha (2.10) está bem definido e pode ser escrito da forma

$$
d s^{2}=g_{\mu \nu} d x^{\mu} d x^{\nu}
$$

onde $g_{\mu \nu}$ representa a métrica do espaço curvo e é uma função das coordenadas $x^{\mu}$. É importante destacar que em um espaço-tempo curvo não é possível definir um referencial de Lorentz global para o qual temos $g_{\alpha \beta}=\eta_{\alpha \beta}$.

\subsection{Geometria Riemanniana}

Na teoria da Relatividade Geral estamos interessados em um tipo especial de variedade diferencial conhecida como variedade Riemanniana, onde a distância ou métrica, estão bem definidas. Logo, esta variedade diferenciável é uma coleção amorfa de pontos, conhecidos como eventos do espaço-tempo. Localmente, estes pontos são ordenados como pontos em um espaço Euclidiano. Ao especificar o conceito de distância, introduzimos a métrica $g_{\mu \beta}$ a qual contém toda a informação de como é a geometria do espaço e como a distância entre os 
pontos é medida.

Consideremos agora, uma curva $\gamma$ em nossa variedade. A curva é parametrizada por $\lambda$ e é descrita em um sistema de coordenadas arbitrário pelas relações $x^{\alpha}(\lambda)$. Ao calcular a razão a qual varia a função escalar $f\left(x^{\alpha}\right)$ ao longo desta curva obtemos

$$
\frac{d f}{d \lambda}=\frac{\partial f}{\partial x^{\alpha}} \frac{d x^{\alpha}}{d \lambda}=f_{, \alpha} u^{\alpha}
$$

$\operatorname{com} f_{, \alpha}=\partial f / \partial x^{\alpha}$ e $u^{\alpha}=d x^{\alpha} / d \lambda$. Logo, ao fazer uma transformação de coordenadas da forma $x^{\alpha} \longrightarrow x^{\alpha^{\prime}}$ temos que a quantidade $d f / d \lambda$ é uma invariante, dado que

$$
f_{, \alpha^{\prime}} u^{\alpha^{\prime}}=f_{, \alpha} u^{\alpha}
$$

onde $f_{, \alpha^{\prime}}=\left(\partial x^{\alpha} / \partial x^{\alpha^{\prime}}\right) f_{, \alpha}$ e $u^{\alpha^{\prime}}=\left(\partial x^{\alpha^{\prime}} / \partial x^{\alpha}\right) u^{\alpha}$. Qualquer objeto $A^{\alpha}$ que transforme como $u^{\alpha}$, em uma transformação de coordenadas

$$
A^{\alpha^{\prime}}=\frac{\partial x^{\alpha^{\prime}}}{\partial x^{\alpha}} A^{\alpha}
$$

será conhecido como vetor. Analogamente, qualquer objeto $A_{\alpha}$ que em uma transformação de coordenadas transforme como $f_{, \alpha}$

$$
A_{\alpha^{\prime}}=\frac{\partial x^{\alpha}}{\partial x^{\alpha^{\prime}}} A_{\alpha}
$$

será denominado de co-vetor ou vetor dual. O fato de vetores e co-vetores se transformarem inversamente sob a mesmas transformações de coordenadas garante que a contração $A^{\alpha} A_{\alpha}$ seja uma invariante. Se generalizamos as definições 2.14 e 2.15 para o caso de um tensor $T_{\gamma \ldots \delta}^{\alpha \ldots \beta}$ do tipo $\left(\begin{array}{c}m \\ n\end{array}\right)$, obtemos a seguinte expressão

$$
T_{\gamma^{\prime} \ldots \delta^{\prime}}^{\alpha^{\prime} \ldots \beta^{\prime}}=\frac{\partial x^{\alpha^{\prime}}}{\partial x^{\alpha}} \ldots \frac{\partial x^{\beta^{\prime}}}{\partial x^{\beta}} \frac{\partial x^{\gamma}}{\partial x^{\gamma^{\prime}}} \ldots \frac{\partial x^{\delta}}{\partial x^{\delta^{\prime}}} T^{\alpha \ldots \beta}{ }_{\gamma \ldots \delta} .
$$

Como a Relatividade Geral é uma teoria covariante devemos construir quantidades que representem as taxas de variação, onde ditas quantidades devem estar definidas em qualquer sistema de coordenadas. Em outras palavras, precisamos definir a derivada, a qual deve ser 
covariante sob uma transformação de coordenadas geral. Para construir o operador derivada, primeiro consideremos a derivada de uma função escalar qualquer $\phi$ definida na variedade Riemanniana. Esta derivada parcial é $\phi_{, \nu} \equiv \partial \phi / \partial x^{\nu}$, a qual transforma como um co-vetor

$$
\phi_{, \nu}^{\prime} \equiv \frac{\partial \phi(x)}{\partial x^{\prime \nu}}=\frac{\partial x^{\mu}}{\partial x^{\prime \nu}} \frac{\partial \phi(x)}{\partial x^{\mu}} \equiv \frac{\partial x^{\mu}}{\partial x^{\prime \nu}} \phi_{, \mu}
$$

Por outro lado, se calculamos a derivada parcial de um vetor qualquer $A^{\alpha^{\prime}}$, obtemos a seguinte expressão

$$
A_{, \beta^{\prime}}^{\alpha^{\prime}}=\frac{\partial}{\partial x^{\beta^{\prime}}}\left(\frac{\partial x^{\alpha^{\prime}}}{\partial x^{\alpha}} A^{\alpha}\right)=\frac{\partial x^{\beta}}{\partial x^{\beta^{\prime}}} \frac{\partial x^{\alpha^{\prime}}}{\partial x^{\alpha}} A_{, \beta}^{\alpha}+\frac{\partial^{2} x^{\alpha^{\prime}}}{\partial x^{\beta} \partial x^{\alpha}} \frac{\partial x^{\beta}}{\partial x^{\beta^{\prime}}} A^{\alpha},
$$

onde $A^{\alpha^{\prime}}$ não é uma transformação tensorial. A existência do segundo termo na equação 2.18 é a razão pela qual o vetor $A^{\alpha^{\prime}}$ não transforma como um tensor. A raiz do problema está no cálculo da derivada a qual envolve a subtração de dois vetores em pontos muito próximos, onde cada um dos vetores em geral obedece uma lei de transformação diferente. Para dar solução a este problema devemos transportar um dos vetores ao ponto mais próximo, de forma tal que a subtração seja feita no mesmo ponto da variedade. Este procedimento é conhecido como Transporte Paralelo, onde as componentes do vetor são transportadas ao longo de uma curva na variedade mantendo constante o ângulo entre os vetores.

Imaginemos que temos um ponto com coordenadas $x^{\mu}$ e seu vizinho mais próximo tem coordenadas $x^{\mu}+d x^{\mu}$. Agora, consideremos o transporte paralelo das componentes do vetor $A_{\alpha}$ desde $x^{\mu}+d x^{\mu}$ até $x^{\mu}$. A derivada do vetor $A^{\alpha}$ será

$$
D A^{\alpha}=d A^{\alpha}+\delta A^{\alpha}
$$

onde $d A^{\alpha}$ é a derivada comum nos pontos $x^{\mu}+d x^{\mu}$ e $x^{\mu}$. Logo, a variação $\delta A^{\alpha}$ contém a regra do transporte paralelo e deve ser uma função linear das componentes originais, $A_{\alpha}$, e do deslocamento $d x^{\mu}$, de modo que podemos escrever

$$
\delta A^{\alpha}=\Gamma_{\gamma \mu}^{\alpha} A^{\gamma} d x^{\mu}
$$

onde $\Gamma_{\alpha \mu}^{\gamma}$ são chamados de Coeficientes de Conexão, e descrevem como os vetores base em 
diferentes pontos da variedade mudam quando um deles se movimenta ao longo da mesma,

$$
\frac{\partial \vec{e}_{\alpha}}{\partial x^{\beta}}=\Gamma_{\alpha \beta}^{\mu} \vec{e}_{\mu}
$$

Assim, a derivada covariante de um vetor é igual a

$$
\nabla_{\beta} A^{\alpha}=A_{; \beta}^{\alpha} \equiv A_{, \beta}^{\alpha}+\Gamma_{\mu \beta}^{\alpha} A^{\mu}
$$

O primeiro termo na equação 2.22 manifesta a variação das componentes do campo vetorial em uma base dada, enquanto que o segundo nos diz a variação devida à mudança dos vetores base ao longo do transporte paralelo. O mesmo procedimento pode der feito para um co-vetor $A_{\alpha}$, obtendo-se a seguinte expressão

$$
\nabla_{\beta} A_{\alpha}=A_{\alpha ; \beta} \equiv A_{\alpha, \beta}-\Gamma_{\alpha \beta}^{\mu} A_{\mu}
$$

O fato que $A_{; \beta}^{\alpha}$ seja um tensor permite-nos deduzir a lei de transformação da conexão $\Gamma_{\gamma \beta}^{\alpha}[8]$, a qual se escreve como

$$
\Gamma_{\gamma^{\prime} \beta^{\prime}}^{\alpha^{\prime}}=\frac{\partial x^{\alpha^{\prime}}}{\partial x^{\alpha}} \frac{\partial x^{\beta}}{\partial x^{\beta^{\prime}}} \frac{\partial x^{\gamma}}{\partial x^{\gamma^{\prime}}} \Gamma_{\gamma \beta}^{\alpha}-\frac{\partial^{2} x^{\alpha^{\prime}}}{\partial x^{\beta} \partial x^{\gamma}} \frac{\partial x^{\beta}}{\partial x^{\beta^{\prime}}} \frac{\partial x^{\gamma}}{\partial x^{\gamma^{\prime}}}
$$

onde o segundo termo nesta lei de transformação revela a natureza não tensorial da conexão. Logo, a diferença entre duas conexões transforma-se como um tensor. Por outro lado, a derivada covariante pode ser estendida a outros tipos de tensor exigindo que o operador obedeça à regra do produto no cálculo diferencial, a regra de Leibniz [1]. Por exemplo, se calculamos a derivada covariante do tensor métrico $g_{\alpha \beta}$ temos

$$
\nabla_{\gamma} g_{\alpha \beta}=g_{\alpha \beta ; \gamma}=g_{\alpha \beta, \gamma}-\Gamma_{\alpha \gamma}^{\mu} g_{\mu \beta}-\Gamma_{\beta \gamma}^{\mu} g_{\alpha \mu}=0
$$

onde $g_{\alpha \beta ; \gamma}$ é identicamente igual a zero, satisfazendo assim o Princípio de Equivalência de Einstein. Logo, ao fazer uma permutação cíclica nos índices da equação 2.25 e assumindo que $\Gamma_{\beta \gamma}^{\alpha}=\Gamma_{\gamma \beta}^{\alpha}$, temos que

$$
g_{\alpha \beta, \gamma}+g_{\alpha \gamma, \beta}-g_{\gamma \beta, \alpha}-2 \Gamma_{\beta \gamma}^{\mu} g_{\alpha \mu}=0
$$


de onde podemos claramente escrever a conexão em termos do tensor métrico

$$
\Gamma_{\beta \gamma}^{\mu}=\frac{1}{2} g^{\mu \alpha}\left(g_{\alpha \beta, \gamma}+g_{\alpha \gamma, \beta}-g_{\gamma \beta, \alpha}\right)
$$

com $g_{\alpha \beta, \gamma}=\partial g_{\alpha \beta} / \partial x^{\gamma}$. As conexões determinadas pela equação 2.27) são conhecidos como Simbolos de Christoffel.

Quando queremos medir a curvatura de uma superfície esférica precisamos somente do raio $a$ da esfera. No entanto, no caso do espaço-tempo a curvatura pode ser descrita pelo tensor $R_{\mu \nu \beta}^{\alpha}$ conhecido como Tensor de Riemann, o qual depende da métrica e suas derivadas. Para descobrir a expressão matemática deste novo tensor consideremos duas partículas de prova, identificadas como 1 e 2, movimentando-se ao longo de suas geodésicas. Logo, denotando como $\xi^{\mu}(\tau)$ a separação infinitesimal entre as partículas no tempo próprio $\tau$ temos que

$$
x_{2}^{\mu}(\tau)=x_{1}^{\mu}(\tau)+\xi^{\mu}(\tau)
$$

onde a linha mundo de cada partícula é descrita pela equação geodésica

$$
\frac{d^{2} x^{\mu}}{d \tau^{2}}+\Gamma_{\alpha \beta}^{\mu}(x) \frac{d x^{\alpha}}{d \tau} \frac{d x^{\beta}}{d \tau}
$$

Ao combinar as equações geodésicas para cada uma das partículas e considerando a expansão em Taylor dos Símbolos de Christoffel, $\Gamma^{\mu}{ }_{\alpha \beta}\left(x_{2}\right)=\Gamma^{\mu}{ }_{\alpha \beta}\left(x_{1}+\xi\right)=\Gamma^{\mu}{ }_{\alpha \beta}\left(x_{1}\right)+$ $\Gamma^{\mu}{ }_{\alpha \beta, \gamma} \xi^{\gamma}$, obtemos a equação para a aceleração de $\xi^{\mu}$

$$
\frac{d^{2} \xi^{\mu}}{d \tau^{2}}+\Gamma_{\alpha \beta}^{\mu} v^{\alpha} \frac{d \xi^{\beta}}{d \tau}+\Gamma_{\alpha \beta}^{\mu} v^{\beta} \frac{d \xi^{\alpha}}{d \tau}+\Gamma_{\alpha \beta, \gamma}^{\mu} \xi^{\gamma} v^{\alpha} v^{\beta}=0
$$

onde usamos $v^{\alpha}=d x^{\alpha} / d \tau$. A equação 2.30 não é uma equação tensorial devido que os Símbolos de Christoffel não transformam como um tensor. Logo, para achar a solução da equação anterior, primeiro devemos calcular a derivada covariante de $\xi^{\mu}$ ao longo da curva geodésica, a qual tem a forma

$$
\frac{D \xi^{\mu}}{D \tau}=\frac{d \xi^{\mu}}{d \tau}+\Gamma_{\alpha \beta}^{\mu} \xi^{\alpha} \frac{d x^{\beta}}{d \tau}
$$


Derivando de novo covariantemente ao londo da geodésica a expressão anterior tendo em consideração a regra do produto para a diferenciação

$$
\frac{d}{d \tau}\left(\Gamma_{\alpha \beta}^{\mu} \xi^{\alpha} v^{\beta}\right)=\Gamma_{\alpha \beta, \gamma}^{\mu} \frac{d x^{\gamma}}{d \tau} \xi^{\alpha} v^{\beta}+\Gamma_{\alpha \beta}^{\mu} \frac{d \xi^{\alpha}}{d \tau} v^{\beta}+\Gamma_{\alpha \beta}^{\mu} \xi^{\alpha} \frac{d v^{\beta}}{d \tau}
$$

Finalmente, obtemos

$$
\frac{D^{2} \xi^{\mu}}{D \tau^{2}}=R_{\alpha \beta \gamma}^{\mu} v^{\alpha} v^{\beta} \xi^{\gamma}
$$

onde

$$
R_{\alpha \beta \gamma}^{\mu}=\Gamma_{\alpha \gamma}^{\sigma} \Gamma_{\sigma \beta}^{\mu}-\Gamma_{\alpha \beta}^{\sigma} \Gamma_{\sigma \gamma}^{\mu}+\Gamma_{\alpha \gamma, \beta}^{\mu}-\Gamma_{\alpha \beta, \gamma}^{\mu},
$$

é conhecido como Tensor de Riemann. Se derivamos parcialmente em relação a $x^{\lambda}$ a equação anterior e avaliamos o resultado em um ponto $P$ de um referencial localmente inercial, obtemos

$$
R_{\alpha \beta \mu \nu, \lambda}=\frac{1}{2}\left(g_{\alpha \nu, \beta \mu \lambda}-g_{\alpha \mu, \beta \nu \lambda}+g_{\beta \mu, \alpha \nu \lambda}-g_{\beta \nu, \alpha \mu \lambda}\right)
$$

onde usamos o fato que no ponto $P$ os Símbolos de Christoffel $\Gamma^{\mu}{ }_{\alpha \beta}$ são nulos, mas sua derivada não. Logo, considerando que o tensor métrico $g_{\alpha \beta}$ é simétrico e que suas derivadas parciais comutam, podemos escrever a conhecida Identidade de Bianchi,

$$
R_{\alpha \beta \mu \nu ; \lambda}+R_{\alpha \beta \lambda \mu ; \nu}+R_{\alpha \beta \nu \lambda ; \mu}=0
$$

O tensor $R_{\alpha \beta \mu \nu}$ é antissimétrico no primeiro e segundo par de índices, e é simétrico no intercambio entre os dois pares. Fazendo a contração $R_{\alpha \beta}=R_{\alpha \mu \beta}^{\mu}=R_{\beta \alpha}$, podemos definir o Escalar de Ricci

$$
R=g^{\mu \nu} R_{\mu \nu}=g^{\mu \nu} g^{\alpha \beta} R_{\alpha \mu \beta \nu}
$$

Finalmente, partindo da equação 2.37 podemos construir o tensor simétrico $G^{\alpha \beta}$

$$
G^{\alpha \beta}=R^{\alpha \beta}-\frac{1}{2} g^{\alpha \beta} R
$$

conhecido como Tensor de Einstein e descreve a curvatura do espaço-tempo nas equações de campo da Relatividade Geral. Este tensor construído a partir do tensor de Riemann e a métrica, satisfaz a condição

$$
\nabla_{\beta} G^{\alpha \beta}=G_{; \beta}^{\alpha \beta}=0
$$


Se segue imediatamente que as Equações de Einstein são iguais a

$$
T_{; \beta}^{\alpha \beta}=G_{; \beta}^{\alpha \beta}=0,
$$

onde as soluções da equação anterior devem ser

$$
G^{\alpha \beta}=\kappa T^{\alpha \beta}
$$

onde a constante $\kappa=8 \pi G / c^{4}$ foi calculada exigindo que devemos recuperar as leis de gravidade newtoniana e da dinâmica no limite de campo gravitacional fraco e movimento não relativístico. Logo, $T^{\alpha \beta}$ representa o tensor de Energia-Momento, o qual é a fonte da curvatura do espaço-tempo. A equação 2.40 representa as leis locais de conservação de energia e momento, garantindo que as perdas de energia e momento de uma região está compensada pelo fluxo de energia e momento fora de dita região.

\subsection{Equações de Einstein pelo Princípio Variacional}

Todas as equações fundamentais da física, incluindo as equações de Einstein, podem ser obtidas a partir do Princípio Variacional. Logo, a condição que deve ser satisfeita para obter as equações de campo é

$$
\delta \int \mathcal{L} d^{4} x=0
$$

onde, obviamente temos uma quantidade invariante, que em nosso caso é escrita em termos da métrica $g_{\mu \beta}$, a qual é uma variável dinâmica na Relatividade Geral. Logo, usando o fato que o único escalar dependente da métrica é o escalar de Ricci $R$, podemos definir a densidade de Lagrangiana como $\mathcal{L}=\sqrt{-g} R$ e escrever a ação como

$$
S_{E H}=\int \sqrt{-g} R d^{4} x
$$

A equação anterior é conhecida como a ação de Einstein-Hilbert [9], dado que as equações de Einstein foram também obtidas por David Hilbert, no ano de 1915, mas usando o princípio de mímina ação de Hamilton. Ao substituir a equação 2.43 na equação 2.42 e fazendo 
uso da expressão (2.37), obtemos

$$
\begin{aligned}
\delta S_{E H} & =\delta \int \sqrt{-g} R d^{4} x \\
& =\int d^{4} x \sqrt{-g} g^{\mu \beta} \delta R_{\mu \beta}+\int d^{4} x \sqrt{-g} \delta g^{\mu \beta} R_{\mu \beta}+\int d^{4} x \delta \sqrt{-g} g^{\mu \beta} R_{\mu \beta} .
\end{aligned}
$$

A variação da ação (2.43) gerou 3 termos, os quais serão analisados por separado. Consideremos o primeiro termo, onde a variação do tensor de Ricci pode ser escrita como

$$
\delta R_{\mu \beta}=\delta \Gamma_{\mu \beta ; \gamma}^{\gamma}-\delta \Gamma_{\mu \gamma ; \beta}^{\gamma}
$$

Se substituímos a equação (2.45) no primeiro termo da expressão (2.44) e lembrando que a derivada covariante da métrica é zero, temos

$$
\begin{aligned}
\int d^{4} x \sqrt{-g} g^{\mu \beta} \delta R_{\mu \beta} & =\int d^{4} x \sqrt{-g} g^{\mu \beta}\left(\delta \Gamma_{\mu \beta ; \gamma}^{\gamma}-\delta \Gamma_{\mu \gamma ; \beta}^{\gamma}\right) \\
& =\int \sqrt{-g} \nabla_{\gamma} J^{\gamma}
\end{aligned}
$$

onde introduzimos o campo vetorial $J^{\gamma}$

$$
J^{\gamma}=g^{\mu \beta} \delta \Gamma_{\mu \beta}^{\gamma}-g^{\mu \gamma} \delta \Gamma_{\mu \beta}^{\beta} .
$$

A integral anterior poder ser resolvida usando o teorema de Stokes, de onde concluímos que a contribução dos termos de superfície no infinito são nulos. Consequentemente, a integral (2.46) fica

$$
\int d^{4} x \sqrt{-g} g^{\mu \beta} \delta R_{\mu \beta}=0
$$

Por outro lado, sabemos que a métrica satisfaz a condição $g_{\mu \beta} g^{\beta \alpha}=\delta^{\alpha}{ }_{\mu}$, logo temos que

$$
\delta \sqrt{-g}=-\frac{1}{2} \sqrt{-g} g_{\mu \beta} \delta g^{\mu \beta}
$$


Assim, a variação da ação de Einstein-Hilbert, torna-se

$$
\begin{aligned}
\delta S_{E H} & =\int d^{4} x \sqrt{-g} R_{\mu \beta} \delta g^{\mu \beta}-\frac{1}{2} \int d^{4} x R \sqrt{-g} g_{\mu \beta} \delta g^{\mu \beta} \\
& =\int d^{4} x \sqrt{-g}\left[R_{\mu \beta}-\frac{1}{2} g_{\mu \beta} R\right] \delta g^{\mu \beta} .
\end{aligned}
$$

Finalmente, usando a equação (2.42), obtemos as Equações de Einstein no vácuo, devido que somente consideramos a parte geométrica da ação

$$
R_{\mu \beta}-\frac{1}{2} g_{\mu \beta} R=0
$$

Para obter as equações de campo completas, vamos considerar que temos presente outro campo além do campo gravitacional. Assim, a ação é escrita da forma

$$
S=\frac{1}{16 \pi G} S_{E H}+S_{M}
$$

onde $S_{M}$ representa a ação da matéria. Seguindo a equação anterior temos finalmente as Equações de Einstein completas

$$
R_{\mu \beta}-\frac{1}{2} g_{\mu \beta} R=\frac{8 \pi G}{c^{4}} T_{\mu \beta}
$$

$\operatorname{com} T_{\mu \beta}$ igual a

$$
T_{\mu \beta}=-2 \frac{1}{\sqrt{-g}} \frac{\delta S_{M}}{\delta g^{\mu \beta}} .
$$

O tensor $T_{\mu \beta}$, contém informação acerca da densidade de energia total medida por um observador inercial arbitrário.

\subsection{Testes clássicos da Relatividade Geral}

Sendo a Relatividade de Einstein uma teoria que alterava radicalmente a maneira de ver o universo, para ser aceita necessitava ser comprovada por medições de precisão que pudessem discernir entre esta nova teoria e a gravitação de Newton. Foi assim que o mesmo Einstein propôs três possíveis testes para a Relatividade Geral: i) O aumento anômalo do periélio de Mercúrio, ii) A deflexão dos raios de luz de estrelas que passam na vizinhança solar, e iii) O 
desvio para o vermelho da luz de origem gravitacional.

\subsubsection{O desvio anômalo do periélio de Mercúrio}

A precessão da órbita de Mercúrio não é peculiar, dado que todas as órbitas dos planetas precessam. De fato, a teoria de Newton prediz estes efeitos, como sendo produzidos pela força gravitacional entre os planetas. A questão é que as predições de Newton concordam com as observações da pressesão das órbitas de quase todos os planetas, exceto a de Mercúrio.

Urbain Jean Joseph Le Verrier tinha sido o primeiro a encontrar provas de uma anomalia na órbita de Mercúrio e também o primeiro em tentar explicar esse efeito. Em setembro de 1859 submeteu à Academia de Ciências de Paris o texto de uma carta a Hervé Faye na qual registrou o que descobriu: o periélio de Mercúrio avança 38" / século devido a alguma ação até então desconhecida [10]. Nessa carta, Le Verrier observava ainda que o único modo de explicar o efeito em termos de corpos conhecidos consistiria em aumentar a massa de Vénus em pelo menos 10\%, modificação inadmissível. Embora duvidasse fortemente, também sugeriu a possibilidade, da existência de um planeta interior a Mercúrio não observado até o momento.

Vista desde a terra, a precessão da órbita de Mercúrio foi medida em 5600" /século. As equações de Newton, considerando os efeitos dos outros planetas, assim como o fato que a terra não é um referencial inercial, prevê uma precessão de 5557"/ século. No entanto, o formalismo Newtoniano não explicava esta discrepância. Em contraste, Einstein foi capaz de predizer, usando o método da aproximação e suas equações de campo, que a órbita de Mercúrio apresenta una precessão extra de 43" / século, marcando assim o início da mecânica celeste pós-Newtoniana.

\subsubsection{A deflexão dos raios de luz de estrelas que passam na vizi- nhança solar}

Einstein também planteou a ideia de que a luz de uma estrela ao passar próxima de um corpo massivo, neste caso o sol, terá sua trajetória alterada, trazendo como consequência que dita luz quando chega à terra teria uma origem aparentemente diferente. 
Este fenômeno foi estudado pela primeira vez no ano de 1919 em duas expedições inglesas, uma a Sobral no Brasil chefiada por Andrew Crommelin do observatório de Greenwich, e outra à ilha do Príncipe chefiada por Arthur Eddington de Cambridge. O seu objetivo era medir a deflexão da luz medida pela Relatividade Geral: 1.75" para um raio de luz tangente ao sol. Eles compararam fotografias feitas das posições de um campo de estrelas, durante um eclipse total do sol, com fotografias do mesmo campo sem a presença do sol. Durante o eclipse, a luz das estrelas observadas era vista apenas após passar por uma zona onde os efeitos gravitacionais do sol são muito fortes. Enquanto que a outra observação das mesmas estrelas foi feita quando os efeitos gravitacionais do sol sobre a luz destas são desprezíveis. Depois de fazer essas comparações, Eddington e Crommelin concluirão que as estrelas apresentam um deslocamento angular causado obviamente pelos efeitos gravitacionais, satisfazendo-se assim as predições feitas pela Relatividade Geral.

Uma das aplicações mais interessantes desta predição é a observação do fenômeno conhecido como lentes gravitacionais, onde a luz proveniente de objetos muito distantes, como gálaxias, a qual está espalhada ao invés de concentrada em um único ponto do céu, sofre diferentes desvios ao passar pelo campo gravitacional de um objeto muito massivo. O resultado disso, será a formação de um arco, ou até mesmo um anel, em torno do objeto massivo.

\subsubsection{O desvio para o vermelho da luz de origem gravitacional}

De acordo com o Princípio de Equivalência da Relatividade Geral, qualquer desvio de frequência proveniente da aceleração de uma fonte radiante, também pode ser produzido por um campo gravitacional específico. Logo, se um fóton de frequência $\nu_{0}$ é emitido radialmente para fora desde a superfície de uma massa gravitacional M, sua energia medida desde uma distância $d$ do corpo será observada como sendo menor, ou desviada ao vermelho.

Em 1959, Pound e Rebka efetuaram pela primeria vez uma experiência baseada neste efeito. Utilizando o efeito Moussbauer, descoberto em 1958, foram capazes de medir a variação da frequência para o trajeto vertical de 22,5 metros da torre do Jefferson Laboratory da Universidade de Harvard. O resultado obtido é compatível com a previsão da Relatividade de Einstein [11]. 
Finalmente, pouco tempo depois de Einstein publicar suas equações de campo da Relatividade Geral, Karl Schwarzschild achou sem muita dificuldade a solução das equações para o caso da geometria do espaço-tempo de uma distribução de matéria esférica, de massa $M$ e completamente estacionaria. Logo, considerando que o espaço exterior da distribução é vazio, Schwarzschild obteve a equação 2.51), onde o tensor energia momento é nulo, reduzindo assim o problema para calcular a métrica $g_{\mu \beta}$ apropriada para o sistema. Desta forma, ele encontrou que o elemento de linha da métrica tem a forma

$$
d s^{2}=-\left(1-\frac{2 G M}{r c^{2}}\right) c^{2} d t^{2}+\frac{d r^{2}}{1-\frac{2 G M}{r c^{2}}}+r^{2} d \theta^{2}+r^{2} \sin ^{2} \theta d \phi^{2} .
$$

Na equação anterior, é possível observar que existe uma singularidade quando o denominador do segundo termo é igual a zero, o qual ocorre quando o raio $r$ associado com o corpo de massa $M$ é igual a $r_{s}=2 G M / c^{2}$. O raio $r_{s}$ é conhecido como Raio de Schwarzschild ou Horizonte de Eventos, dado que os eventos que ocorrem dentro dele não podem propagar sinais de luz para o exterior. Logo, qualquer corpo o suficientemente pequeno para existir dentro de seu próprio horizonte de eventos, está isolado do resto do universo, sendo sua única manifestação de existência o seu forte potencial gravitacional. Nos anos 60, o termo Buraco Negro começou a ser usado para identificar potencias gravitacionais como estes.

Adicionalmente, a métrica de Schwarzschild também pode ser usada para derivar com exatidão tanto o avanço do pericentro da órbita do planeta Mercúrio, assim como a deflexão gravitacional da luz, comprovando novamente a validade da teoria da Relatividade de Einstein. Isto é possível devido ao Teorema de Birkhoff, o qual estabelece que qualquer solução esfericamente simétrica das equaçẽes de campo de Einstein no vácuo devem ser estática e plana assintoticamente. Logo, a solução exterior é esta definida pela métrica de Schwarzschild, equação 2.55). 


\section{Capítulo 3}

\section{Teleparalelismo Equivalente à Relatividade Geral}

O Teleparalelismo equivalente à Relatividade Geral (TEGR), é uma formulação geométrica alternativa à teoria da Relatividade Geral de Einstein, onde os efeitos do campo gravitacional são descritos em termos dos campos de tétradas $e^{a}{ }_{\mu}$, os quais estão definidos no espaço-tempo de Weitzenböck, também conhecido como espaço-tempo de Cartan. Logo, dado um conjunto de tétradas, é possível construir tanto o tensor métrico $g_{\mu \nu}$ como os símbolos de Christoffel ${ }^{0} \Gamma_{\mu \nu}^{\lambda}$, ambos definidos em termos de conexões definidas neste novo espaço-tempo 12 .

As primeiras tentativas para descrever o campo gravitacional usando as tétradas são atribuídas a Einstein no seu esforço para unir a gravitação e o eletromagnetismo [2]. Em 1929, Einstein percebeu que as equações de campo obtidas a partir de uma densidade de Lagrangiana do tipo $\mathcal{L}=e\left(A I_{1}+B I_{2}+C I_{3}\right)$, com $A=1 / 4, B=1 / 2, C=-1$ e $I_{1}, I_{2}, I_{3}$ escritos em termos do tensor de torção, são simétricas nos dois índices livres do espaço-tempo, e que a teoria linearizada resultante descreve o campo gravitacional. Entretanto, usando esta aproximação ele não conseguiu encontrar uma descrição fiel e consistente das equações do campo eletromagnético.

Posteriormente, Møller [13] usando a ideia original de Einstein, encontrou uma quantidade tensorial capaz de descrever a densidade de energia-momento gravitacional, onde dito tensor é invariante sob transformações gerais de coordenadas, mas não é invariante sob transformações 
locais de Lorentz. Logo, baseando-se no trabalho de Møller, Pelegrini e Plebanski [14] calcularam a formulação Lagrangiana da teoria da gravidade teleparalela em termos dos campos de tétradas.

No ano de 1967, Cho comprovou que a densidade de Lagrangiana do teleparalelismo é invariante sob tranformações locais de Lorentz e que é equivalente à densidade de lagrangiana de Einstein-Hilbert da teoria da Relatividade Geral [15]. Em 1979, Hayashi e Shirafuji [16 investigaram em detalhe uma classe geral de teorias da gravidade. Essa nova teoria co-

nhecida como "Nova Relatividade Geral", representava uma forma de adicionar torção à teoria da Relatividade de Einstein. Adicionalmente, eles concluíram que para certas configurações da escolha dos parâmetros constantes, a densidade de Lagrangiana se reduz ao escalar de Curvatura $e R$ da geometria Riemanniana. No mesmo período de tempo, Hehl [17] e Nitsch [18] estudaram a classe geral de teorias da gravidade no contexto da geometria de Riemann-Cartan. Essas são teorias escritas em termos de campos de tétradas e conexões independentes, e que incluem as teorias de teleparalelismo como um caso especial onde uma de ditas teorias é equivalente à Relatividade Geral.

A partir desse momento, múltiplas contribuções foram feitas à teoria, gerando finalmente o que hoje é conhecida como Teleparalelismo Equivalente à Relatividade Geral, ou simplesmente como Gravidade Teleparalela. Entretanto, o estudo de novos aspectos assim como novos descobrimentos, ainda tem que ser feitos nesta teoria.

\subsection{Propriedades básicas do Campo de Tétradas}

Um campo de tétradas é um conjunto de vetores linearmente independentes que obedecem uma relação de ortogonalidade. Esses vetores são usados para construir uma base capaz de descrever um espaço-tempo. A caraterística mais importante das tétradas é que, na descrição do espaço-tempo em termos destes campos, o Princípio da Equivalência surge de maneira natural. Isto é devido ao fato de que os campos de tétradas, os quais descrevem ao mesmo tempo o espaço-tempo físico e o espaço tangente, podem ser interpretados como uma transformação de Lorentz entre os diferenciais $d x^{\mu}$ do espaço-tempo físico e $d q^{a}$ do 
espaço-tempo tangente, através da comparação entre a métrica de Minkowski,

$$
\Lambda^{c}{ }_{a} \Lambda_{b}^{d}{ }_{b} \eta_{c d}=\eta_{a b}
$$

e a métrica do espaço-tempo físico

$$
e^{a}{ }_{\mu}^{b}{ }_{\eta} \eta_{a b}=g_{\mu \eta}
$$

onde $\Lambda_{b}^{a}$ é a matriz de transformação de Lorentz. Logo, sempre é possível escrever as quantidades projetadas

$$
e^{a}=e_{\mu}^{a} d x^{\mu}, \quad d q^{a}=e^{a}{ }_{\mu} d x^{\mu},
$$

muito embora não possamos integrar $d q^{a}$ e escrever $q^{a}=q^{a}\left(x^{\mu}\right)$.

Em um espaço-tempo físico arbitrário, sempre há um espaço-tempo plano tangente em cada ponto, onde o espaço físico será designado pela letra grega $\mu=(0,1,2,3)$ e o espaço tangente será designado pelo índice do grupo $S O(3,1)$ local de Lorentz $a=[(0),(1),(2),(3)]$. Desta forma, o vetor $e^{(0)}{ }_{\mu}(x)$ é do tipo tempo e os vetores $e^{(i)}{ }_{\mu}(x)$ são vetores tipo espaço com $i=(1,2,3) \llbracket 19]$. Os campos de tétradas $e^{a}{ }_{\mu}$ nos permitem fazer a projeção de uma quantidade definida neste espaço-tempo no espaço-tempo tangente. Para isto, consideremos um vetor definido no espaço-tempo $V^{\mu}$. Assim, a correspondente projeção no espaço-tempo tangente é dada pela expressão

$$
V^{a}=e^{a}{ }_{\mu} V^{\mu}
$$

De maneira similar, é possível projetar um vetor definido no espaço-tempo tangente $V^{a}$ no espaço-tempo físico,

$$
V^{\mu}=e_{a}{ }^{\mu} V^{a}
$$

usando o campo de tétradas inverso $e_{a}{ }^{\mu}$. Logo, as quantidades cujas componentes possuem índices do espaço-tempo físico se comportam como tensores sob transformações de coordenadas, enquanto aquelas quantidades cujas componentes possuem índices do espaço-tempo tangente são tensores por transformações de Lorentz. Devido à existência de um campo de 
tétradas inverso, a relação de ortogonalidade entre as tétradas pode ser expressa como

$$
g^{\mu \nu}=e^{a \mu} e_{a}^{\nu}, \quad \eta^{a b}=e^{a \mu} e^{b}{ }_{\mu}
$$

Para construir um campo de tétradas em um espaço-tempo plano, onde obviamente o espaço-tempo tangente será o própio espaço-tempo físico, vamos considerar dois sistemas de coordenadas: o primeiro, $q^{a}=(t, x, y, z)$ no espaço-tempo tangente, e o segundo $x^{\mu}=(t, r, \theta, \phi)$ no espaço-tempo físico. Dado que os dois sistemas estão relacionados pela transformação de coordenadas $d q^{a}=e^{a}{ }_{\mu} d x^{\mu}$, o campo de tétradas pode ser escrito como

$$
e_{\mu}^{a}=\frac{\partial q^{a}}{\partial x^{\mu}}=\left(\begin{array}{cccc}
1 & 0 & 0 & 0 \\
0 & \sin \theta \cos \phi & r \cos \theta \cos \phi & -r \sin \theta \sin \phi \\
0 & \sin \theta \sin \phi & r \cos \theta \sin \phi & r \sin \theta \cos \phi \\
0 & \cos \theta & -r \sin \theta & 0
\end{array}\right) \text {. }
$$

Quando o campo de tétradas está definido como o gradiente da função $q^{a}$, equação (3.7), as tétradas são conhecidas como Holonômicas, onde tanto $x^{\mu}$ quanto $q^{a}$ descrevem os mesmos pontos do espaço-tempo, como é de se esperar em uma transformação de coordenadas [20]. A mesma ideia pode ser usada para construir outras configurações de tétrada, por exemplo, usando uma transformação de coordenadas que se relacionam através de um "boots" de Lorentz.

No caso geral, o campo de tétradas não pode ser escrito na forma $\partial_{\mu} q^{a}$, $\log$ a tétrada é conhecida como não-holônoma. Esta categoria de transformações satisfaz a propriedade $\partial_{\mu} e^{a}{ }_{\nu}-\partial_{\nu} e^{a}{ }_{\mu} \neq 0$, resultando que para um espaço-tempo com torção, as tétradas obedecem a condição de não-holonomicidade.

\subsection{Formulação Lagrangiana}

A descrição do campo gravitacional na teoria do TEGR não é única, dado que a formulação Lagrangiana poder ser desenvolvida utilizando-se tanto uma simetria $S O(3,1)$ local 2123 quanto uma simetria do grupo $S O(3,1)$ global de Lorentz [3,24 26], sendo essas construções 
equivalentes quanto às equações obtidas.

No presente trabalho, exigiremos inicialmente que a teoria exiba invariância local de Lorentz através da introdução de uma conexão $\omega_{\mu a b}$ do grupo $S O(3,1)$ local, para posteriormente impor que essa conexão seja nula, e obter assim uma densidade de Lagrangiana invariante por transformações globais de Lorentz. Partindo da densidade de Lagrangiana as equações de campo podem ser obtidas.

Quando a conexão de spin é introduzida, a condição de Teleparalelismo exige que a derivada covariante da tétrada seja nula, garantindo deste jeito que o campo de tétradas constitua um conjunto de campos auto-paralelos, tendo-se que

$$
\begin{aligned}
\nabla_{\mu} e^{a}{ }_{\nu}=e^{a}{ }_{\nu ; \mu} & =0, \\
\partial_{\mu} e^{a}{ }_{\nu}-{ }^{0} \Gamma_{\mu \nu}^{\lambda} e^{a}{ }_{\lambda}+{ }^{0}{ } \omega_{\mu}{ }^{a}{ }_{b} e^{b}{ }_{\nu} & =0,
\end{aligned}
$$

onde ${ }^{0} \omega_{\mu}{ }^{a}{ }_{b}$ é a conexão de Levi-Civita, a qual é livre de torção e pode ser definida como

$$
{ }^{0} \omega_{\mu a b}=-\frac{1}{2} e^{c}{ }_{\mu}\left(\Omega_{a b c}-\Omega_{b a c}-\Omega_{c a b}\right)
$$

$\operatorname{com} \Omega_{a b c}$ igual a

$$
\Omega_{a b c}=e_{a \nu}\left(e_{b}{ }^{\mu} \partial_{\mu} e_{c}^{\nu}-e_{c}^{\mu} \partial_{\mu} e_{b}^{\nu}\right)
$$

Logo, na teoria do TEGR há uma identidade muito importante, a qual relaciona a conexão de Levi-Civita ${ }^{0} \omega_{\mu a b}$, dada pela equação (3.9), como o tensor de Contorção $K_{\mu a b}$ da forma

$$
{ }^{0} \omega_{\mu a b}=-K_{\mu a b}
$$

onde $K_{\mu a b}$ é definido em termos do tensor de Torsão $T_{\lambda \mu \nu}=e^{a}{ }_{\lambda} T_{a \mu \nu}$,

$$
K_{\mu a b}=\frac{1}{2} e_{a}^{\lambda} e_{b}^{\nu}\left(T_{\lambda \mu \nu}+T_{\nu \lambda \mu}+T_{\mu \lambda \nu}\right)
$$

A identidade (3.11), pode ser obtida por meio de cálculo direto ou através do seguinte procedimento. Primeiro, consideremos uma variedade pseudo-Riemanianna dotada com um campo de tétradas $e^{a}{ }_{\mu}$, e uma conexão $S O(3,1)$ arbitrária $\omega_{\mu a b}$. Ditas quantidades definem 
tanto o tensor de torção como o tensor de curvatura [19], respectivamente, como

$$
\begin{aligned}
\mathcal{T}^{a}{ }_{\mu \nu}(e, \omega) & =\partial_{\mu} e^{a}{ }_{\nu}-\partial_{\nu} e^{a}{ }_{\mu}+\omega_{\mu}{ }^{a}{ }_{b} e^{b}{ }_{\nu}-\omega_{\nu}{ }^{a}{ }_{b} e^{b}{ }_{\mu}, \\
R^{a}{ }_{b \mu \nu}(\omega) & =\partial_{\mu} \omega_{\nu}{ }^{a}{ }_{b}-\partial_{\nu} \omega_{\mu}{ }^{a}{ }_{b}+\omega_{\mu}{ }^{a}{ }_{c} \omega_{\nu}{ }^{c}{ }_{b}-\omega_{\nu}{ }^{a}{ }_{c} \omega_{\mu}{ }^{c}{ }_{b} .
\end{aligned}
$$

Logo, a equação que define $\mathcal{T}_{a \mu \nu}(e, \omega)$ pode ser resolvida para $\omega_{a \mu \nu}$. Deste jeito, fazendo certas manipulações matemáticas e considerando a condição de antissimetria $\omega_{\mu a b}=-\omega_{\mu b a}$, é possível obter a identidade

$$
\omega_{\mu a b}={ }^{0} \omega_{\mu a b}(e)+K_{\mu a b},
$$

onde a conexão $\omega_{\mu a b}$ é conhecida como Conexão de Spin e com $K_{\mu a b}$ definida da forma

$$
K_{\mu a b}=\frac{1}{2} e_{a}^{\lambda} e_{b}{ }^{\nu}\left(\mathcal{T}_{\lambda \mu \nu}+\mathcal{T}_{\nu \lambda \mu}+\mathcal{T}_{\mu \lambda \nu}\right)
$$

Devido que a conexão de spin não exerce influência na dinâmica dos campos de tétradas no TEGR, podemos fazer $\omega_{\mu a b}$ igual a zero na equação (3.15), e obter desta maneira a identidade 3.11 onde $\mathcal{T}_{a \mu \nu}$ se reduz a $T_{a \mu \nu}$.

Sabemos que a derivada covariante de um campo de tétradas, equação (3.8), é identicamente zero e está escrita em termos dos símbolos de Christoffel e a conexão de Levi-Civita. Se multiplicamos todos os termos da expressão 3.8 por $e^{a \lambda}$ obtemos

$$
e^{a \lambda} \partial_{\mu} e_{a \nu}={ }^{0} \Gamma_{\mu \nu}^{\lambda}-e^{a \lambda}\left({ }^{0} \omega_{\mu a b}\right) e^{b}{ }_{\nu}
$$

Logo, ao considerar a identidade (3.11) a expressão anterior fica como

$$
\Gamma_{\mu \nu}^{\lambda}={ }^{0} \Gamma_{\mu \nu}^{\lambda}+e^{a \lambda} K_{\mu a b} e^{b}{ }_{\nu}
$$

onde $\Gamma_{\mu \nu}^{\lambda}=e^{a \lambda} \partial_{\mu} e_{a \nu}$ é conhecido como Conexão de Weitzenböck. Por outro lado, dado que a teoria do TEGR é construída em termos dos campos de tétradas e do tensor de torsão, é possível calcular o tensor de curvatura em termos da conexão de Levi-Civita ${ }^{0} \omega_{\mu a b}$

$$
R_{a b \mu \nu}\left({ }^{0} \omega\right)=\partial_{\mu}{ }^{0} \omega_{\nu a b}-\partial_{\nu}{ }^{0} \omega_{\mu a b}+{ }^{0} \omega_{\mu a c}{ }^{0} \omega_{\nu}{ }^{c}{ }_{b}-{ }^{0} \omega_{\nu a c}{ }^{0} \omega_{\mu}{ }^{c}{ }_{b} .
$$


Usando a equação (3.17) para substituir ${ }^{0} \omega_{\text {mab }}$ na equação anterior, obtemos uma expressão do tensor de curvatura em termos dos campos de tétradas e dos símbolos de Christoffel. Agora, ao multiplicar essa nova expressão pela quantidade $e e^{a \mu} e^{b \nu}$, onde $e$ é o determinante do campo de tétradas, obtemos o escalar de curvatura $R$,

$$
\begin{aligned}
e R(e)= & e e^{a \mu} e^{b \nu} R_{a b \mu \nu}\left({ }^{0} \omega\right) \\
e R(e)= & e e^{a \mu} e^{b \nu}\left\{e_{b}{ }^{\rho} e_{a \sigma} R^{\sigma}{ }_{\rho \mu \nu}\left({ }^{0} \Gamma\right)+{ }^{0} \Gamma^{\sigma}{ }_{\nu \rho}\left[e_{a \sigma}\left(\partial_{\mu} e_{b}{ }^{\rho}\right)+e_{b}{ }^{\rho}\left(\partial_{\mu} e_{a \sigma}\right)\right]\right. \\
& -\left(\partial_{\mu} e_{b}{ }^{\rho}\right)\left(\partial_{\nu} e_{a \rho}\right)+\left(\partial_{\nu} e_{b}{ }^{\rho}\right)\left(\partial_{\mu} e_{a \rho}\right)-{ }^{0} \Gamma^{\sigma}{ }_{\mu \rho}\left[e_{b}{ }^{\rho}\left(\partial_{\nu} e_{a \sigma}\right)+e_{a \sigma}\left(\partial_{\nu} e_{b}{ }^{\rho}\right)\right] \\
& -e_{c}{ }^{\rho} e_{a \sigma} e_{b}{ }^{\lambda}\left[{ }^{0} \Gamma^{\sigma}{ }_{\mu \rho}\left(\partial_{\nu} e^{c}{ }_{\lambda}\right)-{ }^{0} \Gamma^{\sigma}{ }_{\nu \rho}\left(\partial_{\mu} e^{c}{ }_{\lambda}\right)\right] \\
& -e_{b}{ }^{\lambda}\left[{ }^{0} \Gamma^{\rho}{ }_{\nu \lambda}\left(\partial_{\mu} e_{a \rho}\right)-{ }^{0} \Gamma^{\rho}{ }_{\mu \lambda}\left(\partial_{\nu} e_{a \rho}\right)\right] \\
& \left.+e_{c}{ }^{\rho} e_{b}{ }^{\lambda}\left[\left(\partial_{\nu} e_{a \rho}\right)\left(\partial_{\nu} e^{c}{ }_{\lambda}\right)-\left(\partial_{\mu} e_{a \rho}\right)\left(\partial_{\mu} e^{c}{ }_{\lambda}\right)\right]\right\} .
\end{aligned}
$$

Fazendo as contrações dos índices e usando o fato que a métrica satisfaz a condição de $g^{\nu \rho}=e^{b \nu} e_{b}{ }^{\rho}$, enquanto as tétradas satisfazem a condição de $\delta_{\sigma}^{\mu}=e^{a \mu} e_{a \sigma}$, a equação anterior pode ser reescrita como

$$
\begin{aligned}
e R(e)= & e R\left({ }^{0} \Gamma\right)-e e^{a \mu} e^{b \nu}\left(\partial_{\mu} e_{b}{ }^{\rho}\right)\left(\partial_{\nu} e_{a \rho}\right)+e e^{a \mu} e^{b \nu}\left(\partial_{\nu} e_{b}{ }^{\rho}\right)\left(\partial_{\mu} e_{a \rho}\right) \\
& +e e^{a \mu} e_{c}{ }^{\rho} g^{\nu \lambda}\left(\partial_{\mu} e_{a \rho}\right)\left(\partial_{\nu} e^{c}{ }_{\lambda}\right)-e e^{a \mu} e_{c}{ }^{\rho} g^{\nu \lambda}\left(\partial_{\nu} e_{a \rho}\right)\left(\partial_{\mu} e^{c}{ }_{\lambda}\right) .
\end{aligned}
$$

Combinando o segundo e o quinto termo da equação anterior, podemos ver que ditos termos se anulam entre si. Da mesma forma, ao combinar o terceiro e o quarto termo, obtemos novamente zero. Logo, a expressão (3.22) fica da seguinte forma

$$
R(e)=R\left({ }^{0} \Gamma\right),
$$

a partir da qual é possível concluir que o escalar de curvatura escrito em termos da Conexão de Weitzenböck é identicamente zero, $R_{\mu \nu \alpha \beta}(\Gamma)=0$. Adicionalmente, da equação 3.23 é possível ver como sob certas condições a teoria do teleparalelismo converge na teoria da Relatividade Geral de Einstein.

Por outro lado, usando a identidade (3.11) podemos escrever o escalar de curvatura (3.20) 
em termos do tensor de Torsão $T_{a \mu \nu}=\partial_{\mu} e_{a \nu}-\partial_{\nu} e_{a \nu}$,

$$
e R(e)=-e e^{a \mu} e^{b \nu}\left[\partial_{\mu} K_{\nu a b}-\partial_{\nu} K_{\mu a b}+K_{\mu a c} K_{\nu}{ }^{c}{ }_{b}-K_{\nu a c} K_{\mu}{ }^{c}{ }_{b}\right] .
$$

Ao substituir a expressão (3.16) na equação anterior e fazer algumas manipulações matemáticas, obtemos

$$
\begin{aligned}
e R(e)= & -\frac{1}{2} \partial_{\mu}\left[e e^{a \mu} e^{b \nu}\left(T_{a \nu b}+T_{b a \nu}+T_{\nu a b}\right)\right]+\frac{1}{2}\left(T_{a \nu b}+T_{b a \nu}+T_{\nu a b}\right) \partial_{\mu}\left(e e^{a \mu} e^{b \nu}\right) \\
& +\frac{1}{2} \partial_{\nu}\left[e e^{a \mu} e^{b \nu}\left(T_{a \mu b}+T_{b a \mu}+T_{\mu a b}\right)\right]-\frac{1}{2}\left(T_{a \mu b}+T_{b a \mu}+T_{\mu a b}\right) \partial_{\nu}\left(e e^{a \mu} e^{b \nu}\right) \\
& -\frac{1}{4} e e^{a \mu} e^{b \nu}\left(T_{a \mu c}+T_{c a \mu}+T_{\mu a c}\right)\left(T^{c}{ }_{\nu b}+T_{b}{ }^{c}{ }_{\nu}+T_{\nu}{ }^{c}{ }_{b}\right) \\
& +\frac{1}{4} e e^{a \mu} e^{b \nu}\left(T_{a \nu c}+T_{c a \nu}+T_{\nu a c}\right)\left(T^{c}{ }_{\mu b}+T_{b}{ }^{c}{ }_{\mu}+T_{\mu}{ }^{c}{ }_{b}\right) .
\end{aligned}
$$

Logo, fazendo as respectivas contrações dos índices com as tétradas e utilizando as propriedades de simetria e antissimetria do tensor de Torção, a expressão anterior pode ser simplificada da forma

$$
\begin{aligned}
e R(e)= & -\frac{1}{4} e T_{a b c} T^{a b c}-\frac{1}{2} e T_{a b c} T^{b a c}+e T^{a} T_{a}+2 \partial_{\mu}\left(e T^{\mu}\right) \\
& +\frac{1}{2}\left(T_{a \nu b}+T_{b a \nu}+T_{\nu a b}\right) \partial_{\mu}\left(e e^{a \mu} e^{b \nu}\right) \\
& -\frac{1}{2}\left(T_{a \mu b}+T_{b a \mu}+T_{\mu a b}\right) \partial_{\nu}\left(e e^{a \mu} e^{b \nu}\right) .
\end{aligned}
$$

Para resolver os termos que contêm derivada parcial da quantidade $e e^{a \mu} e^{b \nu}$, primeiro devemos considerar que dita quantidade pode ser chamada de tensor de Densidade $\phi^{a \mu b \nu}$ com peso $\omega$. Logo, ao calcular a derivada covariante desta densidade ao longo de uma geodésica obtemos

$$
\phi^{a b \mu \nu}{ }_{j}=\partial_{\nu} \phi^{a b \mu \nu}+{ }^{0} \Gamma^{\mu}{ }_{\lambda \nu} \phi^{a b \lambda \nu}+{ }^{0} \Gamma^{\nu}{ }_{\lambda \nu} \phi^{a b \mu \lambda}-\omega^{0} \Gamma^{\lambda}{ }_{\lambda \nu} \phi^{a b \mu \nu}=0 .
$$

Se usamos a equação (3.27) para escrever a derivada parcial $\partial_{\nu} \phi^{a b \mu \nu}$ em termos dos 
símbolos de Christoffel, a expressão 3.26 pode ser reescrita como

$$
\begin{aligned}
e R(e)= & -\frac{1}{4} e T_{a b c} T^{a b c}-\frac{1}{2} e T_{a b c} T^{b a c}+e T^{a} T_{a}+2 \partial_{\mu}\left(e T^{\mu}\right) \\
& +\frac{1}{2}\left(T_{a \nu b}+T_{b a \nu}+T_{\nu a b}\right) \partial_{\mu}\left(e e^{a \mu} e^{b \nu}\right) \\
& -\frac{1}{2}\left(T_{a \mu b}+T_{b a \mu}+T_{\mu a b}\right)\left(-{ }^{0} \Gamma_{\lambda \mu}^{\nu} \phi^{a b \mu \lambda}-{ }^{0} \Gamma_{\lambda \mu}^{\mu} \phi^{a b \lambda \nu}+\omega^{0} \Gamma_{\lambda \mu}^{\lambda} \phi^{a b \mu \nu}\right) .
\end{aligned}
$$

onde $T^{a}=T^{b}{ }_{b}{ }^{a}$. Ao fazer a mundança de índices $\mu \longleftrightarrow \nu$ e $a \longleftrightarrow b$ no quinto termo da expressão anterior, e aplicando as propriedades de antissimetria, temos

$$
+\frac{1}{2}\left(T_{a \nu b}+T_{b a \nu}+T_{\nu a b}\right) \partial_{\mu}\left(e e^{a \mu} e^{b \nu}\right)=-\frac{1}{2}\left(T_{a \nu b}+T_{b a \nu}+T_{\nu a b}\right) \partial_{\mu}\left(e e^{b \nu} e^{a \mu}\right) .
$$

Desta maneira, se subtraímos o termo da expressão anterior ao último termo da equação (3.28), obtemos explicitamente a derivada covariante mostrada na equação (3.27). Portanto, a contribuição destes termos no escalar de curvatura $e R(e)$ é nula. Finalmente, a expressão (3.28) fica da forma

$$
e R(e)=-e\left(\frac{1}{4} T_{a b c} T^{a b c}+\frac{1}{2} T^{a b c} T_{b a c}-T^{a} T_{a}\right)+2 \partial_{\mu}\left(e T^{\mu}\right)
$$

Por outro lado, como já conhecemos a forma exata do escalar de curvatura escrito em termos do tensor de torção, podemos escrever a densidade de Lagrangiana $\mathcal{L}$ do TEGR. Para isso, primeiro devemos introduzir o tensor $\Sigma^{a b c}$ definido em 27

$$
\Sigma^{a b c}=\frac{1}{4}\left(T^{a b c}+T^{b a c}-T^{c a b}\right)+\frac{1}{2}\left(\eta^{a c} T^{b}-\eta^{a b} T^{c}\right) .
$$

Com o tensor 3.31 podemos escrever a seguinte combinação quadrática

$$
\Sigma^{a b c} T_{a b c}=\frac{1}{4} T^{a b c} T_{a b c}+\frac{1}{2} T^{a b c} T_{b a c}-T^{a} T_{a}
$$

Logo, a equação 3.30 pode ser reescrita como

$$
e R(e)=-e \Sigma^{a b c} T_{a b c}+2 \partial_{\mu}\left(e T^{\mu}\right)
$$

Para escrever a densidade de Lagrangiana podemos desprezar o termo $\partial_{\mu}\left(e T^{\mu}\right)$, dado 
que ao construir a integral da ação para espaços-tempo assintoticamente planos, as integrais de superfície que surgem da intregração por partes se anulam. Finalmente, a densidade de Lagrangiana é dada pela expressão [19

$$
\mathcal{L}(e)=-k e \sum^{a b c} T_{a b c}-\frac{1}{c} \mathcal{L}_{M}
$$

onde $\mathcal{L}_{M}$ representa a densidade de Lagrangiana do campo de matéria, e $k=c^{3} / 16 \pi G$ ou $k=1 / 16 \pi$ quando as unidades naturais são consideradas. O passo a seguir, uma vez conhecida a densidade de Lagrangiana é obter as equações de campo a partir da variação funcional da equação 3.34 em relação à tétrada $e_{a \mu}$,

$$
\begin{aligned}
\delta \mathcal{L}(e) & =0 \\
& =\delta\left(-e k \Sigma^{a b c} T_{a b c}\right)-\delta \mathcal{L}_{M}, \\
& =-k(\delta e) \Sigma^{a b c} T_{a b c}-k e\left(\delta \Sigma^{a b c}\right) T_{a b c}-e k \Sigma^{a b c}\left(\delta T_{a b c}\right)+e T^{a \mu} \delta e_{a \mu},
\end{aligned}
$$

onde $T^{a \mu}$ é definido por $\delta \mathcal{L}_{M}=e T^{a \mu} \delta e_{a \mu}$. A variação em relação à tétrada gerou 4 termos, os quais devem ser resolvidos separadamente. Consideremos o primeiro termo da equação 3.35, onde $\delta e=e e^{d \beta} \delta e_{d \beta}$, temos que

$$
-k(\delta e) \Sigma^{a b c} T_{a b c}=-k e e^{d \beta} \Sigma^{a b c} T_{a b c} \delta e_{d \beta} .
$$

Ao fazer a seguinte mudança de índices $a \rightarrow b, b \rightarrow c, c \rightarrow d$ e dividindo pela quantidade $\delta e_{a \mu}$, obtemos

$$
\begin{aligned}
-k e e^{d \beta} \Sigma^{b c d} T_{b c d} \frac{\delta e_{d \beta}}{\delta e_{a \mu}} & =-k e e^{d \beta} \Sigma^{b c d} T_{b c d} \delta_{d}^{a} \delta_{\beta}^{\mu} \\
& =-k e e^{a \mu} \Sigma^{b c d} T_{b c d}
\end{aligned}
$$

Agora, consideremos o segundo termo da equação 3.35 , onde $\Sigma^{a b c}$ vem dado pela ex- 
pressão (3.31). Logo, temos que

$$
\begin{aligned}
-k e\left(\delta \Sigma^{a b c}\right) T_{a b c} & =-k e \delta\left[\frac{1}{4}\left(T^{a b c}+T^{b a c}-T^{c a b}\right)+\frac{1}{2}\left(\eta^{a c} T^{b}-\eta^{a b} T^{c}\right)\right] T_{a b c} \\
& =-k e\left[\frac{1}{4}\left(\delta T^{a b c}+\delta T^{b a c}-\delta T^{c a b}\right)+\frac{1}{2}\left(\eta^{a c} \delta T^{b}-\eta^{a b} \delta T^{c}\right)\right] T_{a b c} \\
& =-k e\left[\frac{1}{4} T_{a b c}\left(\delta T^{a b c}+\delta T^{b a c}-\delta T^{c a b}\right)+\frac{1}{2} T_{a b c}\left(\eta^{a c} \delta T^{b}-\eta^{a b} \delta T^{c}\right)\right] .
\end{aligned}
$$

Fazendo algumas mudanças de índices e considerando as propriedades de antissimetria do tensor de torção, a expressão anterior pode ser reescrita da forma

$$
\begin{aligned}
-k e\left[\frac{1}{4} T_{a b c}\left(\delta T^{a b c}+\delta T^{b a c}-\delta T^{c a b}\right)+\frac{1}{2} T_{a b c}\left(\eta^{a c} \delta T^{b}-\eta^{a b} \delta T^{c}\right)\right] & =-k e \Sigma_{a b c}\left(\delta T^{a b c}\right) \\
& =-k e \Sigma^{a b c}\left(\delta T_{a b c}\right)
\end{aligned}
$$

Dado que a equação (3.39) é igual ao terceiro termo da expressão (3.35), estes podem ser somados, obtendo-se o seguinte resultado

$$
-2 k e \Sigma^{a b c}\left(\delta T_{a b c}\right)
$$

Logo, para encontrar uma solução para a equação anterior, devemos lembrar que o tensor de torção pode ser escrito como $T_{a \mu \nu}=\partial_{\mu} e_{a \nu}-\partial_{\nu} e_{a \mu}$, com o qual é possível fazer a seguinte transformação,

$$
T_{a b c}=e_{b}{ }^{\mu} e_{c}{ }^{\nu} T_{a \mu \nu}
$$

Ao substituir a expressão anterior, na equação (3.40), temos

$$
\begin{aligned}
-2 k e \Sigma^{a b c}\left(\delta T_{a b c}\right)= & -2 k e \Sigma^{a b c} \delta\left(e_{b}{ }^{\mu} e_{c}{ }^{\nu} T_{a \mu \nu}\right) \\
= & -2 k e \Sigma^{a b c}\left(\delta e_{b}{ }^{\mu}\right) e_{c}{ }^{\nu} T_{a \mu \nu}-2 k e \Sigma^{a b c} e_{b}{ }^{\mu}\left(\delta e_{c}{ }^{\nu}\right) T_{a \mu \nu} \\
& -2 k e \Sigma^{a b c} e_{b}{ }^{\mu} e_{c}{ }^{\nu}\left(\partial_{\mu} \delta e_{a \nu}-\partial_{\nu} \delta e_{a \mu}\right) .
\end{aligned}
$$

Usando a expressão

$$
\delta e_{b}{ }^{\mu}=-e_{b}^{\beta} e^{d \mu} \delta e_{d \beta},
$$


a equação 3.42 pode ser reescrita como

$$
\begin{aligned}
& -2 k e \Sigma^{a b c}\left(-e_{b}{ }^{\beta} e^{d \mu} \delta e_{d \beta}\right) e_{c}{ }^{\nu} T_{a \mu \nu}-2 k e \Sigma^{a b c} e_{b}{ }^{\mu}\left(-e_{c}{ }^{\beta} e^{d \nu} \delta e_{d \beta}\right) T_{a \mu \nu} \\
& -2 k e \Sigma^{a b c} e_{b}{ }^{\mu} e_{c}{ }^{\nu}\left(\partial_{\mu} \delta e_{a \nu}-\partial_{\nu} \delta e_{a \mu}\right) .
\end{aligned}
$$

Agora, consideremos a derivada

$$
\partial_{\mu}\left(e \Sigma^{a b c} e_{b}^{\mu} e_{c}^{\nu} \delta e_{a \nu}\right)=e \Sigma^{a \mu \nu} \partial_{\mu}\left(\delta e_{a \nu}\right)+\delta e_{a \nu} \partial_{\mu}\left(e \Sigma^{a \mu \nu}\right),
$$

a qual é nula na superfície de integração. Logo, a expressão anterior fica como

$$
e \Sigma^{a \mu \nu} \partial_{\mu}\left(\delta e_{a \nu}\right)=-\delta e_{a \nu} \partial_{\mu}\left(e \Sigma^{a \mu \nu}\right)
$$

Substituindo a expressão anterior na equação (3.44), obtemos

$$
\begin{aligned}
& 2 k e \Sigma^{a b c} e_{b}{ }^{\beta} e_{c}{ }^{\nu} e^{d \mu} T_{a \mu \nu} \delta e_{d \beta}+2 k e \Sigma^{a b c} e_{b}{ }^{\mu} e_{c}{ }^{\beta} e^{d \nu} T_{a \mu \nu} \delta e_{d \beta} \\
& +2 k \delta e_{a \nu} \partial_{\mu}\left(e \Sigma^{a \mu \nu}\right)-2 k \delta e_{a \mu} \partial_{\nu}\left(e \Sigma^{a \mu \nu}\right) .
\end{aligned}
$$

Fazendo primeiro a contração dos índices com as tétradas na equação anterior, e depois a mudança $\mu \rightarrow \lambda$ e $a \rightarrow b$, temos

$$
\begin{aligned}
& 2 k e \Sigma^{b \beta \nu} e^{d \lambda}\left(\delta e_{d \beta}\right) T_{b \lambda \nu}+2 k e \Sigma^{b \lambda \beta} e^{d \nu}\left(\delta e_{d \beta}\right) T_{b \lambda \nu} \\
& +2 k\left(\delta e_{b \nu}\right) \partial_{\lambda}\left(e \Sigma^{b \lambda \nu}\right)-2 k\left(\delta e_{b} \lambda\right) \partial_{\nu}\left(e \Sigma^{b \lambda \nu}\right) .
\end{aligned}
$$

Se dividimos a equação 3.48 por $\delta e_{a \mu}$, e fazemos algumas manipulações matemáticas, obtemos

$$
-4 k\left[\partial_{\lambda}\left(e \Sigma^{a \mu \lambda}\right)-e \Sigma^{b \lambda \mu} T_{b \lambda}^{a}\right]
$$

Finalmente, podemos escrever as equações de campo a partir da variação de $\mathcal{L}(e)[19$

$$
\begin{aligned}
0 & =-k e e^{a \mu} \Sigma^{b c d} T_{b c d}-4 k\left[\partial_{\lambda}\left(e \Sigma^{a \mu \lambda}\right)-e \Sigma^{b \lambda \mu} T_{b \lambda}{ }^{a}\right]+e T^{a \mu} \\
\frac{1}{4 k} e T^{a \mu} & =\partial_{\lambda}\left(e \Sigma^{a \mu \lambda}\right)-e\left(\Sigma^{b \lambda \mu} T_{b \lambda}{ }^{a}-\frac{1}{4} e^{a \mu} \Sigma^{b c d} T_{b c d}\right) .
\end{aligned}
$$


Apesar de que a densidade de Lagrangiana não é invariante sob uma transformação $S O(3,1)$ arbitrária, as equações de campo 3.50 são covariantes sob uma transformação local do grupo $S O(3,1)$.

A teoria definida pela equação (3.34) é equivalente à Relatividade Geral de Einstein, devido ao fato que é possível demonstrar que o lado direito da equação (3.50) poder ser reescrito como

$$
R^{a \mu}(e)-\frac{1}{2} e^{a \mu} R(e)=\frac{1}{2 k} e T^{a \mu}
$$

justificando desta maneira o próprio nome da teoria. Essa equivalência pode ser visualizada quando analisamos a forma como a Relatividade Geral é descrita. Usualmente, ela é formulada em termos de um tensor de curvatura diferente de zero, o que a faz uma teoria essencialmente geométrica, e com o tensor de torção nulo. Logo, para a teoria do Teleparalelismo a cena é oposta, mas absolutamente equivalente. Tem-se a curvatura construída a partir da conexão de Cartan nula, e a torção diferente de zero [20].

Por outro lado, reescrevendo a equação 3.50 obtemos

$$
\partial_{\lambda}\left(e \Sigma^{a \mu \lambda}\right)=\frac{1}{4 k} e T^{a \mu}+e\left(\Sigma^{b \lambda \mu} T_{b \lambda}{ }^{a}-\frac{1}{4} e^{a \mu} \Sigma^{b c d} T_{b c d}\right)
$$

Definindo o tensor

$$
t^{a \mu}=k\left(4 \Sigma^{b \lambda \mu} T_{b \lambda}^{a}-e e^{a \mu} \Sigma^{b c d} T_{b c d}\right),
$$

conhecido como tensor de Energia-Momento gravitacional [28], a equação (3.52) fica da forma,

$$
\partial_{\lambda}\left(e \Sigma^{a \mu \lambda}\right)=\frac{1}{4 k} e\left(t^{a \mu}+T^{a \mu}\right)
$$

ou ainda,

$$
\partial_{\lambda}\left(e \Sigma^{a \mu \lambda}\right)=\frac{1}{4 k} e e_{\lambda}^{a}\left(t^{\lambda \mu}+T^{\lambda \mu}\right) .
$$

Dado que o tensor $\Sigma^{a \mu \nu}$ é antissimétrico nos dois últimos índices, $\Sigma^{a \mu \nu}=-\Sigma^{a \nu \mu}$, se satisfaz que $\partial_{\mu} \partial_{\lambda}\left(e \Sigma^{a \mu \lambda}\right)=0$. Logo, temos que

$$
\partial_{\mu}\left[e e_{\lambda}^{a}\left(t^{\lambda \mu}+T^{\lambda \mu}\right)\right]=0,
$$


a qual pode ser interpretada como uma lei de conservação local para o tensor de energiamomento gravitacional $t^{a \mu}$, e o tensor de campos de matéria $T^{a \mu}$. Usando a expressão (3.56) é possível escrever a equação de Continuidade para o campo gravitacional

$$
\frac{d}{d t} \int_{V} d^{3} x e e_{\mu}^{a}\left(t^{0 \mu}+T^{0 \mu}\right)=-\oint_{S} d S_{j}\left[e e^{a}{ }_{\mu}\left(t^{j \mu}+T^{j \mu}\right)\right]
$$

onde as integrações são feitas sob um volume $V$ e uma superfície $S$ que envolve $V$. Dado que o tensor $t^{a \mu}$ é o tensor de energia-momento gravitacional, o lado direito da equação (3.57) é interpretado como o quadri-vetor de energia-momento total contida em um volume $V$ e com componentes $P^{a}=(E / c, \mathbf{P})$,

$$
P^{a}=\int_{V} d^{3} x e e^{a}{ }_{\mu}\left(t^{0 \mu}+T^{0 \mu}\right) .
$$

\subsection{Interpretação do Campo de Tétradas}

As invariâncias exibidas pela densidade de Lagrangiana, 3.34 são responsáveis pela interpretação do campo de tétradas como sistemas de referência. Desta maneira, a invariância da teoria por transformações globais $S O(3,1)$ estabelece que se dois campos de tétradas que são soluções das equações de campo, produzem o mesmo tensor métrico e que não se relacionam por nenhuma transformação global de Lorentz, ditos campos descrevem dois sistemas de referência diferentes. Logo, o significado físico desses objetos é que eles representam sistemas de referência adaptados a observadores ideais de massa nula no espaço-tempo.

Cada conjunto de tétradas define uma classe de sistemas de referência. Logo, se denotamos por $x^{\mu}(s)$ a linha mundo $C$ de um observador no espaço-tempo, e por $u^{\mu}(s)=d x^{\mu} / d s$ sua velocidade ao longo de $C$, podemos fazer a identificação da velocidade do observador como a componente $a=(0)$ do campo de tétradas $e_{a}{ }^{\mu}$. A aceleração do observador é dada por $a^{\mu}=D u^{\mu} / d s=D e_{(0)}{ }^{\mu} / d s=u^{\alpha} \nabla_{\alpha} e_{(0)}{ }^{\mu}$, onde a derivada covariante é escrita em termos dos símbolos de Christoffel.

Vemos, que a tétrada $e_{a}^{\mu}$ determina a velocidade e a aceleração, ao longo de uma linha mundo de um observador adaptado a um sistema de referência. Deste ponto de vista, po- 
demos concluír que um conjunto de tétradas para o qual $e_{(0)}^{\mu}$ descreve uma congruência de curvas do tipo tempo, é adaptado a uma classe de observadores. A título de comparação, devemos lembrar que se $e^{a}{ }_{\mu} \rightarrow \delta^{a}{ }_{\mu}$ no limite $r \rightarrow \infty$, $\log 0 e^{a}{ }_{\mu}$ é adaptado a observadores estáticos no infinito espacial.

Podemos generalizar a noção de aceleração no espaço-tempo por meio da derivada absoluta da tétrada,

$$
\frac{D e_{a}{ }^{\mu}}{d s}=\phi_{a}{ }^{b} e_{b}^{\mu}
$$

onde $\phi_{a}{ }^{b}$ é o tensor de acelerações antissimétrico. Seguindo o que é apresentado em [30], em analogia ao tensor de Faraday podemos identificar $\phi_{a b} \rightarrow(\mathbf{a}, \boldsymbol{\Omega})$, onde a é a aceleração translacional, $\phi_{(0)(i)}=a_{(i)}$, e $\boldsymbol{\Omega}$ é a frequência de rotação de um sistema de referência em relação a outro que não está em rotação (transporte de Fermi-Walker [31]).

A equação 3.59 pode ser invertida, obtendo-se

$$
\phi_{a}{ }^{b}=e^{b}{ }_{\mu} \frac{D e_{a}{ }^{\mu}}{d s}=e^{b}{ }_{\mu} u^{\lambda} \nabla_{\lambda} e_{a}{ }^{\mu},
$$

onde a derivada covariante é escrita em termos dos símbolos de Christoffel. Dado que esta conexão se relaciona com a conexão de spin definida em (3.15) por meio da equação (3.8), ao substituir dita conexão na expressão acima, $\phi_{a b}$ fica

$$
\phi_{a b}=\frac{1}{2}\left[T_{(0) a b}+T_{a(0) b}-T_{b(0) a}\right]
$$

onde $\phi_{a b}$ pode ser interpretada como a aceleração inercial ao longo de $x^{\mu}(s)$. Logo, dado um conjunto de tétradas em um espaço-tempo arbitrário, sua interpretação geométrica pode ser obtida tanto pela adequada identificação da velocidade $u^{\mu}=c e_{(0)}{ }^{\mu}$ do campo de observadores, junto com a orientação no espaço tridimensional das componentes $e_{(1)}^{\mu}, e_{(2)}^{\mu}, e_{(3)}{ }^{\mu}$, ou pelos valores do tensor aceleração $\phi_{a b}=-\phi_{b a}$, o qual caracteriza o estado inercial do referencial. Logo, a condição $e_{(0)}^{\mu}=u^{\mu} / c$ fixa somente as três componentes $e_{(1)}^{\mu}, e_{(2)}^{\mu}, e_{(3)}^{\mu}$, devido que a componente $e_{(0)}^{\mu}$ é determinada pela condição de normalização $u^{\mu} u^{\nu} g_{\mu \nu}=-c^{2}$. Em ambos casos, a fixação do referencial vai depender da fixação de seis componentes do campo de tétradas. 


\section{Capítulo 4}

\section{Einstein Toolkit}

A teoria da Relatividade Geral, apesar de ser uma teoria conceitualmente simples e elegante, na prática resulta ser uma teoria extremamente complexa. As equações de campo conformam um sistema de dez equações diferenciais parciais em quatro dimensões, acopladas e não lineares. Logo, ditas equações escritas em sua forma geral possuem milhares de termos. Devido a esta complexidade, somente são conhecidas soluções exatas sob condições de alta simetria, esférica ou axial, além de soluções estáticas, estacionarias e homogêneas. Se estamos interessados em estudar situações com muita relevância astrofísica que envolvam campos gravitacionais intensos, dinâmicos e com pouca ou nenhuma simetria, resulta impossível encontrar uma solução exata. Da necessidade de estudar esses sistemas surgiu a Relatividade Numérica, a qual procura calcular uma solução para as equações de Einstein fazendo uso de aproximações numéricas.

A Relatividade Numérica foi desenvolvida na década dos anos sessenta como um campo de investigação independente, começando com os esforços de Hahn e Lindquist [32] para encontrar numericamente a solução das equações de Einstein para um sistema binário de buracos negros. Naquela época o poder dos computadores era muito limitado e as simulações que podiam ser feitas se limitavam a problemas com simetria esférica ou axial e com resolução muito baixa. No entanto, essa situação mudou nas décadas dos anos oitenta e noventa devido a uma revolução no campo da Relatividade Númerica. Em 1999, foi reportada a primeira simulação completamente relativística de um sistema binário de estrelas de nêutrons em fusão [33]. Posteriormente, em 2005 foi executada com sucesso a primeira simulação de um sistema de dois 
buracos negros [34], onde sob certas condições de resolução, o sistema evolui tempo suficiente para gerar informação sobre a órbita de fusão e as ondas gravitacionais emitidas durante o evento.

Logo, simultaneamente aos avanços tanto de nossa compreensão física da dinâmica relativística, assim como das técnicas numéricas necessárias para estudá-las, um conjunto geral de ferramentas e bibliotecas computacionais têm sido desenvolvido com o objetivo de fornecer uma base computacional que pudesse proporcionar uma nova ciência, facilitando a pesquisa colaborativa e interdisciplinar, além de promover a reutilização de softwares aproveitando a infraestrutura computacional avançada (supercomputadores) emergente: surge assim o Einstein Toolkit.

O Einstein Toolkit (ET) consiste em um conjunto de numerosas componentes cuja maioria utilizam o ambiente computacional conhecido como Cactus Toolkit, o qual fornece uma infraestrutura modular básica para simulações numéricas. Um conjunto de ferramentas são providas por este ambiente de trabalho, incluindo desde o controle das simulações, passando pela análise de dados até sua visualização final [35]. A seguir faremos uma introdução ao Cactus assim como daquelas ferramentas que consideramos mais importantes para auxiliar o entendimento de novos usuários. Outras ferramentas mais específicas ao uso do programa ET serão posteriormente descritas.

\subsection{Visão Estrutural}

\subsubsection{Cactus}

O código Cactus foi inicialmente desenvolvido pela Louisiana State University, embora a ideia original tivesse sido concebida pelo Albert Einstein Institute em conjunto com o National Center for Supercomputing Applications. Este ambiente foi criado para aplicações modulares, onde os programas são separados em componentes, chamados "thorns", com interação e dependências claramente definidas [36,37]. Os thorns são tipicamente desenvolvidos

de forma independente e podem ser intercambiáveis com outros de mesma funcionalidade. Normalmente eles não interagem diretamente entres si, ao contrário eles interagem por meio 
de uma base comum conhecida como "flesh". Adicionalmente, os thorns podem ser escritos em diferentes linguagens de programação como C, C++, Fortran, OpenCL e CUDA.

As simulações realizadas pelo Cactus requerem um arquivo executável para poderem ser compiladas, o qual é tipicamente feito pelos próprios usuários. A configuração estabelecida neste executável permite rápidas adaptações às mudanças no ambiente local, mas principalmente funciona como uma maneira de modificar o código fonte conforme à necessidade do usuário. Logo, o executável sempre pode ser compilado a partir de um arquivo de texto ou de parâmetros, onde são definidos tanto os thorns que serão utilizados, como o conjunto de valores que os parâmetros dos thorns assumirão (os parâmetros não definidos assumirão valores padrão), portanto cada executável estabelece uma simulação específica. Porém um executável que contenha todos os thorns pode ser usado para modelar uma variedade de cenários físicos. Não existe restrição alguma para o nome do arquivo de parâmetros, embora seja necessário usar a extensão .par. Adicionalmente, a primeira linha de comandos neste arquivo de deve ser o ActiveThorns, o qual é um comando especial que especifica ao programa quais dos thorns serão implementados na simulação, dado que por omisão todos os thorns estão inativos.

Um termo comumente utilizado no Cactus é a Função Grid. Esta função representa a discretização de uma variável definida em todos os pontos de um grid. Desta maneira, podemos considerar como exemplos desta função a densidade de massa em repouso de um fluido definida em todos os pontos de um determinado domínio, e as componentes do tensor métrico da Relatividade Geral de Einstein.

\subsubsection{Carpet}

O Cactus separa os códigos físicos da infraestrutura computacional, ou seja, aqueles thorns que implementam as equações de Einstein ou as equações da magnetohidrodinâmica relativística (MHD) não contêm nenhuma sentença que faça referência à administração da memória, à paralelização, ao tempo de evolução ou o Input/ Output (OI). A maioria destas tarefas estão contidas em um thorn especial, conhecido como Driver thorn ou somente Driver. 
O ET fornece dois drivers: o PUGH e o Carpet. O PUGH implementa um grid uniforme com topologia cartesiana, enquanto o Carpet [38] proporciona mais que um grid unidimensional: ele implementa o "Refinamento Adaptativo de malhas" (Adaptative mesh refinement-AMD), o qual utiliza o algoritmo padrão de Berger-Oliger [39]. Atualmente, o Carpet é o principal driver utilizado para realizar simulações astrofísicas baseadas no Cactus.

\subsubsection{SimFactory}

O SimFactory ou o Simulation Factory [40], é um conjunto de ferramentas projetadas para gerenciar diferentes tarefas necessárias para a configuração e execução de simulações numéricas no Cactus. Fazendo uso destas ferramentas, a maioria das operações são simplificadas e muitos tipos de erros comuns aos usuários, são evitados. Desta forma, o SimFactory permite ao usuário desenvolver o código localmente, e depois sincronizar e compilar em todas as outras maquinas possíveis, com simples comandos. Além disso, ele mantém um registro da localização e quais simulações foram executadas.

Para entender o funcionamento do SimFactory, consideremos o seguinte cenário: um usuário do ET executa todo o desenvolvimento de seu código em um notebook, onde algumas simulações simples e de baixa resolução podem ser compiladas. Para fazer outras simulações de maior complexidade, o usuário poderia utilizar um computador com melhores componentes, o que implicaria na sincronização, compilação e execução dos arquivos e parâmetros do código, analisando ao final o output. Logo, para casos de alta resolução o usuário poderia ter de recorrer aos clusters, onde os processos de sincronização, compilação,

executação e análise teriam de ser repetidos. É perceptível que este processo pode facilmente levar a erros.

\subsubsection{GetComponents}

Como mencionamos anteriormente, o ET é composto por um conjunto de muitos componentes independentes (Cactus, Carpet e muitos thorns), os quais tipicamente estão localizados em repositórios distintos, com diferentes versões de sistemas de controle e que são mantidos por diferentes grupos. O GetComponents fornece uma forma unificada de baixar todos estes componentes, evitando desta maneira maiores complicações aos usuários. 
o GetComponents é um script com somente um argumento obrigatório, conhecido como Thornlist. A thornlist é um arquivo de texto simples que contém a lista dos thorns a serem baixados, a sua localização e a versão do sistema de controle de cada um desse thorns. Logo, a thornlist pode ser dada como um URL ou especificada com o nome do arquivo de texto.

\subsubsection{Anatomia de um Thorn}

Qualquer Cactus thorn tem a seguinte estrutura que pode ser visualizada através do caminho de arquivos ./Cactus/arrangements/

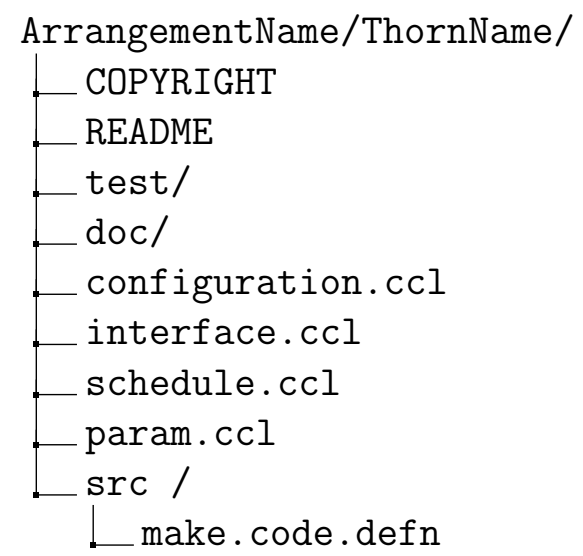

onde a pasta doc/ contém a documentação relacionada com o thorn. Na pasta par/ é possível encontrar alguns exemplos dos arquivos de parâmetros para o uso do thorn. Por sua vez, na src/ se encontra o arquivo make.code.defn o qual é necessário para a compilação dos arquivos do thorn.

Os quatro arquivos com extensão .ccl servem para especificar a interface do thorn com o Cactus flesh, onde três deles são obrigatórios e um é opcional. O arquivo opcional é o configuration.ccl, o qual mostra a dependência entre os thorns no processo de compilação. O interface.ccl é um arquivo utilizado para definir as variáveis e as funções grid do thorn, assim como as funções compartilhadas entre os mesmos. Adicionalmente, este arquivo contém toda a informação referente à relação existente entre os thorns, os arquivos usados procedentes de outros thorns e uma lista das variáveis globais deles. O arquivo schedule.ccl gerencia a função scheduling e o armazenamento global das funções grid. Finalmente, o param.ccl define todos os parâmetros e estabelece seus valores por omissão. 


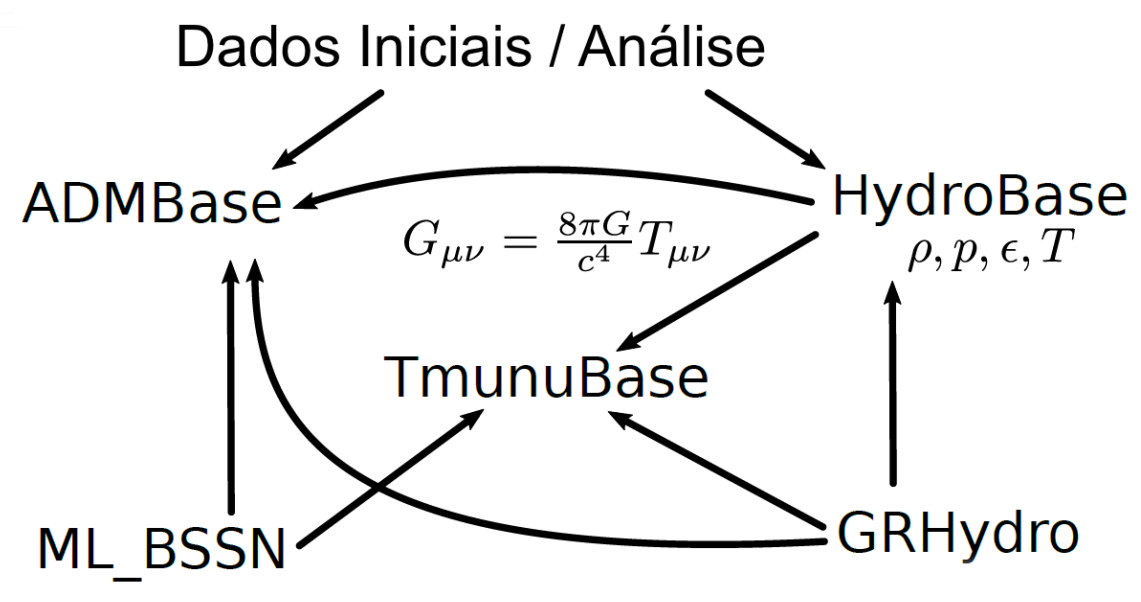

Figura 4.1: Visão geral dos thorns centrais do arrangement EinsteinBase: ADMBase, HydroBase e TmunuBase em conjunto com alguns thorns escolhidos pelo usuário. Neste caso, os thorns do usuários são o ML_BSSN e o GRHydro usados respectivamente na evolução da curvatura e da magetohidrodinâmica 35].

\subsection{Principais thorns}

Antes de começar com a descrição de alguns dos principais thorns implementados no ET, é importante fazer referência aos arrangements, os quais são conjuntos de thorns que usualmente compartilham a mesma funcionalidade ou tem origem em comum. Estes grupos foram criados para auxiliar aos usuários no entendimento e uso dos thorns, e não acrescentam significado especial a eles. A seguir, faremos uma introdução de alguns dos arrangements básicos do ET assim como de seus principais thorns.

\subsubsection{EinsteinBase}

Esta coleção define e registra as variáveis básicas da Relatividade Númerica. Os thorns que fazem uso ou modificam as variáveis ADM [41], por exemplo, devem utilizar este arrangement ao invés de definir seus próprios thorns. Na figura 4.1 apresentamos um esquema que detalha as relações típicas entre estes e outros thorns definidos pelos usuários de acordo com a simulação pretendida por eles. A maioria dos thorns relacionados à física da Relatividade são baseados nestes códigos. É de grande importância para novos usuários terem pelo menos uma visão geral da estrutura dos seguintes thorns básicos. 
1. ADMBase. O ET proporciona o código de evolução das equações de campo de Einstein $G^{\mu \nu}=8 \pi T^{\mu \nu}$. Logo, os métodos relativísticos de evolução usados no ambiente Cactus utilizam diferentes formalismos, os quais estão baseados principalmente na construção 3+1 ADM [41]. Nesta aproximação, o espaço-tempo é concebido como uma sequência de hipersuperfícies 3-dimensionais tipo espaço, conectadas por vetores normais tipo tempo. A divisão $3+1$ introduz quatro graus de liberdade de calibre, definidos pela função lapse $\alpha$, a qual descreve a evolução do tempo próprio de um observador normal em relação à coordenada tempo $\bigsqcup^{1}$ e pelo vetor de desvio $\beta^{i}$, que descreve a variação das coordenadas espaciais desde uma hipersuperfície para a seguinte.

De acordo com a formulação ADM, a métrica do espaço-tempo tem a seguinte forma

$$
d s^{2}=g_{\mu \nu} d x^{\mu} d x^{\nu} \equiv\left(-\alpha^{2}+\beta^{i} \beta_{i}\right) d t^{2}+2 \beta_{i} d t d x^{i}+\gamma_{i j} d x^{i} d x^{j}
$$

onde $g_{\mu \nu}, \alpha, \beta^{i}$ e $\gamma_{i j}$ são respectivamente a métrica 4-dimensional do espaço-tempo, a função lapse, o vetor de desvio e a métrica 3-dimensional do espaço. É assumida a convenção padrão dos índices, onde as letras latinas são usadas para representar os índices espaciais 3-dimensionais, enquanto as letras gregas são utilizadas na representação do espaço-tempo 4-dimensional, e onde os índices vão desde 0 a 3 . Logo, o tensor de curvatura extrínseca $K_{i j}$ é definido em termos da derivada temporal da métrica e da derivada de Lie com respeito ao vetor de desvio [39],

$$
K_{i j}=-\frac{1}{2 \alpha}\left(\partial_{t}-\mathcal{L}_{\beta}\right) \gamma_{i j}
$$

Desta maneira, as variáveis definidas no ADMBase são

- O tensor métrico 3-dimensional $\gamma_{i j}$ : gxx, gxy, gxz, gyy, gyz, gzz

- O tensor de curvatura extrínseca $K_{i j}$ : kxx, kxy, kxz, kyy, kyz, kzz

- A função lapse $\alpha$ : alp

- O vetor de desvio $\beta^{i}$ : betax, betay, betaz

\footnotetext{
${ }^{1}$ Um observador normal segue uma linha mundo tangente ao vetor normal da hipersuperfície 3-dimensional
} 
Os valores iniciais das simulações feitas no ET são determinados pelos parâmetros seguintes: o inicial_data, o qual estabelece os dados para a métrica 3-dimensional e a curvatura extrínseca, enquanto o initial_lapse e o initial_shift estabelecem respectivamente os valores iniciais da função lapse e do vetor de desvio. Adicionalmente, as derivadas temporais das variáveis de calibre, são determinadas pelos parâmetros initial_dtlapse e initial_dtshift. O ADMBase inicializa a métrica 3-dimensional e a curvatura extrísenca com os valores do espaço-tempo de Minkowski, ou seja, $\gamma_{i j}=\delta_{i j}$ e $K_{i j}=0$, o vetor de desvio $\beta^{i}=0$ e a função lapse $\alpha=1$. Entretanto, os thorns encarregados dos valores iniciais os substituem através de seus parâmetros. É importante ressaltar que o ADMBase em si mesmo não executa nem a evolução da métrica 3-dimensional, nem da curvatura $K_{i j}$, enquanto mantêm a função lapse e o vetor $\beta^{i}$ estáticos. Os métodos encarregados de realizar a evolução são determinados pelos seguintes parâmetros: o evolution_method estabelecido para a métrica 3-dimensional e a curvatura extrínseca, o lapse_evolution_method e o shift_evolution_method, os quais estabelecem os métodos de evolucão da função $\alpha$ e do vetor $\beta^{i}$, assim como de suas derivadas temporais dtlapse_evolution_method e dtshift_evolution_method.

2. HydroBase. Similarmente ao ADMBase, o HydroBase estabelece bases comuns para a interação entre os thorns relacionados com problemas evolutivos, mais especificamente no caso da hidrodinâmica relativística. O HydroBase inclui no ET uma interface na qual a magnetohidrodinâmica possa funcionar. A principal função deste thorn é armazenar as variáveis conhecidas como variáveis primitivas, as quais são usadas na maioria dos códigos que envolvem solução das equações de Euler. Essas também são as variáveis necessárias no acoplamento resolutivo do espaço-tempo, assim como são frequentemente necessárias para realizar análises em outros thorns. Juntamente com o ADMBase, o uso comum de um conjunto de variáveis por diferentes códigos hidrodinâmicos, cria a possibilidade de compartilhar partes entre os códigos, por exemplo, as rotinas de análise de dados. As variáveis primitivas definidas pelo HydroBase são 39]

- rho: densidade de massa em repouso $\rho$

- press: pressão $P$ 
- eps: densidade de energia interna $\epsilon$

- vel [3]: velocidade 3-dimensional do fluido $v^{i}$, definida en termos da quadrivelocidade $u^{i}$, a função lapse e o vetor de desvio

$$
v^{i}=\frac{u^{i}}{\alpha u^{0}}+\frac{\beta^{i}}{\alpha}
$$

- Y_e: fração de elétrons $Y_{e}$

- temperature: temperatura $T$

- entropy: entropia especifica por partícula $s$

- Bvec [3]: vetor de campo magnético definido em termos do tensor de Faraday dual $F^{* \mu \nu}=1 / 2 \epsilon^{\mu \nu \alpha \beta} F_{\alpha \beta}$ e a unidade normal da foliação do espaço-tempo $n^{\mu} \equiv$ $\alpha^{-1}\left[1,-\beta^{i}\right]^{\mu}$

$$
B^{i}=\frac{1}{\sqrt{4 \pi}} n_{\nu} F^{* \nu i}
$$

Adicionalmente, o Hydrobase constrói blocos de agendamento que organizam as principais funções que os thorns da hidrodinâmica possam precisar. Todos estes blocos são opcionais, mas quando utilizados eles simplificam os códigos existentes e fazem deles mais interoperáveis.

3. TmunuBase. No ET, o tensor energia-momento $T_{\mu \nu}$ é considerado para o caso de um fluido ideal relativístico,

$$
T^{\mu \nu}=\rho h u^{\mu} u^{\nu}-g^{\mu \nu} P
$$

onde $\rho$ é a densidade de massa em repouso, $u^{\mu}$ é a quadri-velocidade do fluido, $h=1+\epsilon+$ $P / \rho$ é entalpia relativística específica, com $P$ e $\epsilon$ como a pressão e a densidade de energia interna, respectivamente. O thorn TmunuBase proporciona funções grid que auxiliam no cálculo do tensor energia-momento $T^{\mu \nu}$, além de grupos de agendamento os quais controlam quando dito cálculo vai ser feito. Logo, em uma simulação, diferentes thorns podem contribuir no cálculo do tensor, onde este thorn em particular permite a eles fazê-lo sem uma interdependência explícita. O tensor energia-momento resultante pode ser usado pelo thorn de evolução do espaço-tempo, novamente sem uma dependência explícita entre os thorns. As funções grid fornecidas pelo thorn são 
- A componente temporal $T_{00}$ : eTtt

- As componentes misturadas $T_{0 i}$ : eTtx, eTty, eTtz

- As componentes espaciais $T_{i j}$ : eTxx, eTxy, eTxz, eTyy, eTyz, eTzz.

\subsubsection{EinsteinInitial}

O ET contém muitos thorns utilizados para gerar os dados iniciais para simulações da Relatividade Geral, onde são incluídas as configurações do vácuo e da hidrodinâmica. Este tipo de arrangements estabelece as condições iniciais para sistemas simples ou binários de buracos negros ou estrelas de nêutrons. Um dos thorns mais utilizados é o seguinte:

1. TOVSolver. Este código resolve as equações Tolman-Oppenheimer-Volkov [42,43] para uma estrela estática, esfericamente simétrica e em equilibro hidrostático. A solução destas equações proporciona os dados iniciais para uma estrela TOV, onde dita solução é expressa em termos das coordenadas de Schwarzschild com $r$ como raio interior da estrela. Logo, as equações do equilíbrio hidrostático são

$$
\begin{aligned}
\frac{d P}{d r} & =-(e+P) \frac{m+4 \pi r^{3} P}{r(r-2 m)}, \\
\frac{d m}{d r} & =4 \pi r^{2} e, \\
\frac{d \Phi}{d r} & =\frac{m+4 \pi r^{3} P}{r(r-2 m)},
\end{aligned}
$$

onde $e \equiv \rho(1+\epsilon)$ é a densidade de energia do fluido, a qual conta com a contribução da energia interna, $m$ é a massa gravitacional contida na esfera de raio $r$, e $\Phi$ é o logaritmo da função lapse $\alpha$. A rotina também proporciona a solução analiticamente conhecida para o exterior da estrela

$$
\begin{aligned}
P & =\text { TOV_atmosphere, } \\
m & =M, \\
\Phi & =\frac{1}{2} \log \left(1-\frac{2 M}{r}\right),
\end{aligned}
$$

onde TOV_atmosphere é um parâmetro usado para definir a densidade da atmosfera da estrela. Dado que o raio isotrópico $\bar{r}$ é a escolha mais comum para iniciar os cálculos 
dinâmicos, logo o código transforma todas as variáveis para as coordenadas isotrópicas. Como resultado desta transformação obtemos uma equação diferencial adicional em termos de $\bar{r}(r)$,

$$
\frac{d(\log (\bar{r} / r))}{\partial r}=\frac{r^{1 / 2}-(r-2 m)^{1 / 2}}{r(r-2 m)^{1 / 2}}
$$

No exterior da estrela, $r>R$, a massa $M \equiv m(R)$ é uma constante, desta maneira a equação 4.12 pode ser resolvida analiticamente já seja para $\bar{r}(r)$ ou $r(\bar{r})$, obtendo-se as seguintes expressões 4

$$
\begin{aligned}
\bar{r}(r) & =\frac{1}{2}\left(\sqrt{r^{2}-2 M r}+r-M\right), \\
r(\bar{r}) & =\bar{r}\left(1+\frac{M}{2 \bar{r}}\right)^{2} .
\end{aligned}
$$

Para facilitar a construção de estrelas em configurações dinâmicas mais complicadas, talvez seja necessário aplicar uma velocidade uniforme à estrela de nêutrons, devido que isso não afeta a solução do sistema de equações diferenciais ordinárias nem o resultado do perfil de densidade resultante.

\subsubsection{EinsteinEvolve/McLachlan}

Este arragenment está formado por múltiplos thorns usados na evolução numérica das equações de campo de Einstein. Os principais thorns deste arragenments são:

\section{McLachlan.}

O ET contém o código de evolução conhecido como McLachlan [44 46], gerado a partir de equações tensoriais fazendo uso do Kranc [47], o qual é uma aplicação do Mathematica que converte um sistema de equações diferenciais parciais com descrição continua de alto nível em um módulo do Cactus altamente otimizado. Ele utiliza um método muito preciso de diferenciação finita até a oitava ordem aos correspondentes termos de dissipação de Kreiss-Oliger para discretizar desta maneira as variáveis do espaço-tempo do formalismo BSSN [48,49]. O McLachaln foi projetado para operar através das interfaces dos thorns ADMBase e TmunuBase. As variáveis envolvidas no cálculo são: o fator conforme $\phi$, a métrica conforme 3 -dimensional $\tilde{\gamma}_{i j}$, o traço $K$ da 
curvatura extrínseca, o traço livre de curvatura $A_{i j}$ e as funções de conexão conforme $\tilde{\Gamma}^{i}$. Logo, estas variáveis estão definidas em termos da métrica 4-dimensional $g_{i j}$, a métrica 3-dimensional $\gamma_{i j}$ e a curvatura extrínseca $K_{i j}$ da forma

$$
\begin{aligned}
\phi & \equiv \log \left[\frac{1}{12} \operatorname{det}\left(\gamma_{i j}\right)\right], \\
\tilde{\gamma}_{i j} & \equiv e^{-4 \phi} \gamma_{i j}, \\
K & \equiv g^{i j} K_{i j}, \\
\tilde{A}_{i j} & \equiv e^{-4 \phi}\left[K_{i j}-\frac{1}{3} g_{i j} K\right], \\
\tilde{\Gamma}^{j} & \equiv \tilde{\gamma}^{j k} \tilde{\Gamma}^{i}{ }_{j k} .
\end{aligned}
$$

Por outro lado, as equações de evolução podem ser escritas da seguinte maneira, onde foi introduzida a notação $\partial_{0}=\partial_{t}-\beta^{j} \partial_{j}[39]$,

$$
\begin{aligned}
\partial_{0} \alpha= & -\alpha^{2} f\left(\alpha, \phi, x^{\mu}\right)\left[K-K_{0}\left(x^{\mu}\right)\right] \\
\partial_{0} K= & -e^{-4 \phi}\left[\tilde{D}^{i} \tilde{D}_{i} \alpha+2 \partial_{i} \phi \cdot \tilde{D}^{i} \alpha\right]+\alpha\left(\tilde{A}^{i j} \tilde{A}_{i j}+\frac{1}{3} k^{2}\right)-\alpha S \\
\partial_{0} \beta^{i}= & \alpha^{2} G\left(\alpha, \phi, x^{\mu}\right) B^{i} \\
\partial_{0} B^{i}= & -e^{-4 \phi} H\left(\alpha, \phi, x^{\mu}\right) \partial_{0} \tilde{\Gamma}^{i}-\eta^{i}\left(B^{i}, \alpha, x^{\mu}\right), \\
\partial_{0} \phi= & -\frac{\alpha}{6} K+\frac{1}{6} \partial_{k} \beta^{k} \\
\partial_{0} \tilde{\gamma}_{i j}= & -2 \alpha \tilde{A}_{i j}+2 \tilde{\gamma}_{k(i} \partial_{j)} \beta^{k}-\frac{2}{3} \tilde{\gamma}_{i j} \partial_{k} \beta^{k}, \\
\partial_{0} \tilde{A}_{i j}= & e^{-4 \phi}\left[\alpha \tilde{R}_{i j}+\alpha R^{\phi}{ }_{i j} \tilde{D}_{i} \tilde{D}_{j} \alpha+4 \partial_{(i} \phi \cdot \tilde{D}_{j)} \alpha\right]^{T F} \\
& +\alpha K \tilde{A}_{i j}-2 \alpha \tilde{A}_{i k} \tilde{A}_{j}^{k}+2 \tilde{A}_{k(i} \partial_{j)} \beta^{k}-\frac{2}{3} \tilde{A}_{i j} \partial_{k} \beta^{k}-\alpha e^{-4 \phi} \hat{S}_{i j}, \\
& \\
\partial_{0} \tilde{\Gamma}^{i}= & \tilde{\gamma}^{k l} \partial_{k} \partial_{l} \beta^{i}+\frac{1}{3} \tilde{\gamma}^{i j} \partial_{j} \partial_{k} \beta^{k}+\partial_{k} \tilde{\gamma}^{k j} \cdot \partial_{j} \beta^{i}-\frac{2}{3} \partial_{k} \tilde{\gamma}^{k i} \cdot \partial_{j} \beta^{j} \\
& +2 \alpha\left[(m-1) \partial_{k} \tilde{A}^{k i}-\frac{2 m}{3} \tilde{D}^{i} K+m\left(\tilde{\Gamma}_{k l}^{i} \tilde{A}^{k l}+6 \tilde{A}^{i j} \partial_{j} \phi\right)\right] \\
& -2 \tilde{A}^{i j} \partial_{j} \alpha-S^{i} .
\end{aligned}
$$


onde a quantidade $S$ vem escrita em termos do tensor de energia-momento $T_{\mu \nu}$

$$
\begin{aligned}
S & \equiv g^{i j} T_{i j} \\
S_{i} & \equiv-\frac{1}{\alpha}\left(T_{0 i}-\beta^{j} T_{i j}\right) .
\end{aligned}
$$

Em particular, $\tilde{D}_{i}$ e $\tilde{\Gamma}^{k}{ }_{i j}$ se referem à derivada covariante e aos símbolos de Christoffel, respectivamente, ambos escritos em termos de $\tilde{\gamma}_{i j}$. A forma $[\ldots]^{T F}$ estabelece a expressão livre de traço contida nos colchetes. Adicionalmente, temos que o tensor de Ricci está definido como

$$
\begin{aligned}
\tilde{R}_{i j} & =-\frac{1}{2} \tilde{\gamma}^{k l} \partial_{k} \partial_{l} \tilde{\gamma}_{i j}+\tilde{\gamma}_{k i(} \partial_{j} \tilde{\Gamma}^{k}-\tilde{\Gamma}_{(i j) k} \partial_{l} \tilde{\gamma}^{l k}+\tilde{\gamma}^{l s}\left(2 \tilde{\Gamma}^{k}{ }_{l(i} \tilde{\Gamma}_{j) k s}+\tilde{\Gamma}^{k}{ }_{i s} \tilde{\Gamma}_{k l j}\right), \\
{ }^{\phi} R_{i j} & =-2 \tilde{D}_{i} \tilde{D}_{j} \phi-2 \tilde{\gamma}_{i j} \tilde{D}^{k} \tilde{D}_{k} \phi+4 \tilde{D}_{i} \phi \tilde{D}_{j} \phi-4 \tilde{\gamma}_{i j} \tilde{D}^{k} \phi \tilde{D}_{k} \phi .
\end{aligned}
$$

Os parâmetros de calibre $f, G, H$ estão definidos pelas seguintes expressões

$$
\begin{aligned}
f\left(\alpha, \phi, x^{\mu}\right) & \equiv 2 / \alpha, \\
K_{0}\left(x^{\mu}\right) & \equiv 0, \\
G\left(\alpha, \phi, x^{\mu}\right) & \equiv(3 / 4) \alpha^{-2}, \\
H\left(\alpha, \phi, x^{\mu}\right) & \equiv \exp \{4 \phi\}, \\
\eta\left(B^{i}, \alpha, x^{\mu}\right) & \equiv(1 / 2) B^{i} q(r) .
\end{aligned}
$$

As equações (4.34), 4.35 e 4.36) dependem da condição de desvio $\Gamma$-driver, a qual é atenuada pela função $q(r)$. Logo, este thorn usa o método de amortecimento simples definido em [50] como

$$
q(r) \equiv\left\{\begin{array}{lll}
1 & \text { para } r \leq R, & \text { (perto da origem) } \\
R / r & \text { para } r \geq R, & \text { (muito longe da origem) }
\end{array}\right.
$$

com a constante $R$ definindo a transição entre o raio interior, onde $q \approx 1$, e o raio exterior, onde $q$ cai rapidamente como $1 / r$. 
2. GRHydro. Este é um código completamente relativístico e hidrodinâmico em três dimensões, baseado na versão pública do código Whisky [4]. Este thorn usa as variáveis definidas no HydroBase além de proporcionar suas próprias hydro variáveis conservativas e os métodos para evoluí-las. Dado que este thorn não fornece nenhuma informação sobre os valores iniciais ou as equações de estado, é preciso utilizar outros thorns que tenham essa função. Por outro lado, o GRHydro usa o thorn MoL para a fazer a evolução temporal. Isso significa que se houver mais thorns evolutivos utilizando este mesmo código, a evolução temporal vai ser feita somente pelo MoL, proporcionando ao GRHydro uma precisão de até a quarta ordem nas variáveis temporais. Para a formulação das equações gerais de estado, este thorn usa a interface EOS_Omni, a qual fornece todas as variáveis hidrodinâmicas necessárias para a simulação. O parâmetro GRHydro_eos_table controla qual das equações de estado vai ser implementada na evolução.

\subsubsection{EinsteinAnalysis}

O EinsteinAnalysis contém múltiplos thorns usados para análise dos dados. Os principais thorns que compõem este arrangement são:

1. ADMAnalysis. Este thorn proporciona rotinas para calcular as seguintes quantidades

- O traço da curvatura extrínseca: trk

- O determinante da métrica: detg

- As componentes da métrica e da curvatura extrínseca em coordenadas esféricas: grr, grq, grp, gqq, gqp, gpp, Krr, Krq, Krp, Kqq, Kqp Kpp

- As componentes do tensor de Ricci: Ricci11, Ricci12, Ricci13, Ricci22, Ricci23, Ricci33

- O escalar de Ricci: Ricci.

2. Multipole. O Multipole é um thorn que expande qualquer função grid do Cactus em termos dos harmônicos esféricos de um raio $r$ dado. Esta rotina é utilizada em combinação como o thorn WeylScal4 para facilitar a expanção dos escalares de Weyl, os quais são usados frequentemente na Relatividade Numérica [35]. Logo, uma função 
grid $u(t, r, \theta, \varphi)$ pode ser expandida em termos dos harmônicos esféricos com peso $s$ da seguinte forma

$$
u(t, r, \theta, \varphi)=\sum_{l=0}^{\infty} \sum_{m=-l}^{l} C^{l m}(t, r){ }_{s} Y_{l m}(\theta, \varphi)
$$

onde os coeficientes $C^{l m}(t, r)$ vem dados pela expressão

$$
C^{l m}(t, r)=\int{ }_{s} Y_{l m}^{*} u(t, r, \theta, \varphi) r^{2} d \Omega
$$

Para um tempo $t$ dado, este thorn pode calcular os coeficientes $C^{l m}(t, r)$ de um número de funções grid em coordenadas esféricas com diferentes raios $r_{i}$. Logo, os coeficientes $C^{l m}$ podem ser encontrados em arquivos de texto . asc cujos nomes tem a seguinte forma mp_<var>_l<lmode>m<mmode>rrrad>.asc, por exemplo, mp_u_12_m2_r10.00.asc.

3. WeylScal4. Este thorn está encarregado de calcular o escalar de Weyl $\Psi_{4}$, utilizado no contexto do formalismo de Newman-Penrose. O escalar de Weyl, definido em um referencial apropriado, pode ser usado para codificar a saída da radiação gravitacional de um sistema assimptoticamente plano, tornando-se desta maneira uma quantidade muito utilizada na análise de ondas gravitacionais.

\subsection{Implementação do Einstein Toolkit}

\subsubsection{Requerimentos}

O ET e o Cactus deveriam funcionar na maioria das variantes de Unix, onde os requerimentos básicos necessários são: compiladores de C, C++, Fortran 90, implementações MPI, HDF5, Perl, Python, curl e gnuplot. Adicionalmente, dado que o ET também pode ser

obtido desde seus próprios repositórios, se faz necessário o uso das ferramentas Subversion e git. 


\subsubsection{Execução do ET}

Para baixar o ET, primeiro devemos obter o GetComponents, que pode ser acessado pelas seguintes linhas de comando:

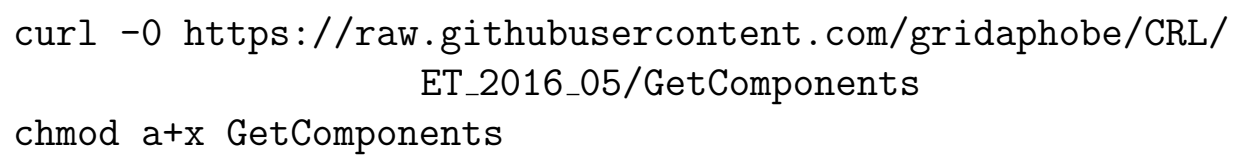

Anteriormente mencionamos que o GetComponents aceita uma lista de thorns como argumento, assim essa lista pode ser obtida usando o procedimento a seguir:

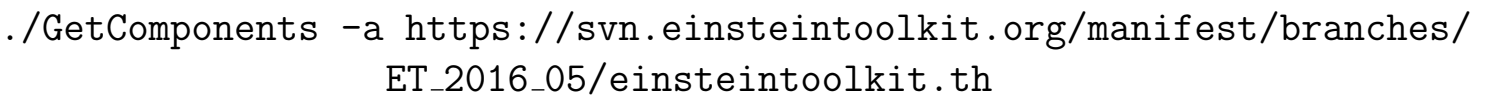

Dado que estamos baixando o arquivo einsteintoolkit.th diretamente desde o repositório original usando o GetComponents, é possível fazer atualizações do código da seguinte forma:

./GetComponents -a $-\mathrm{u}$./einsteintoolkit.th

A seguir apresentamos a estrutura típica de uma thornlist

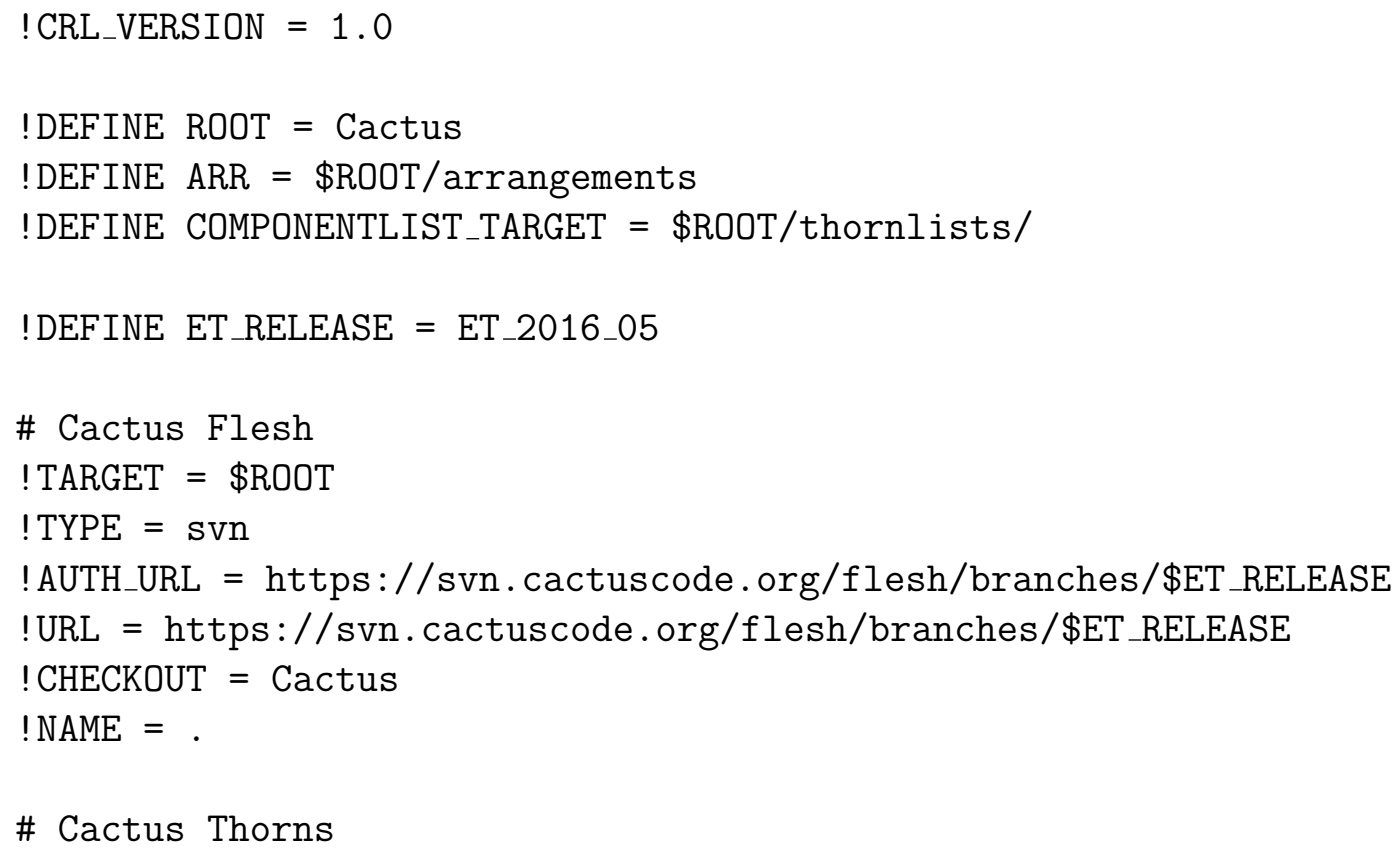




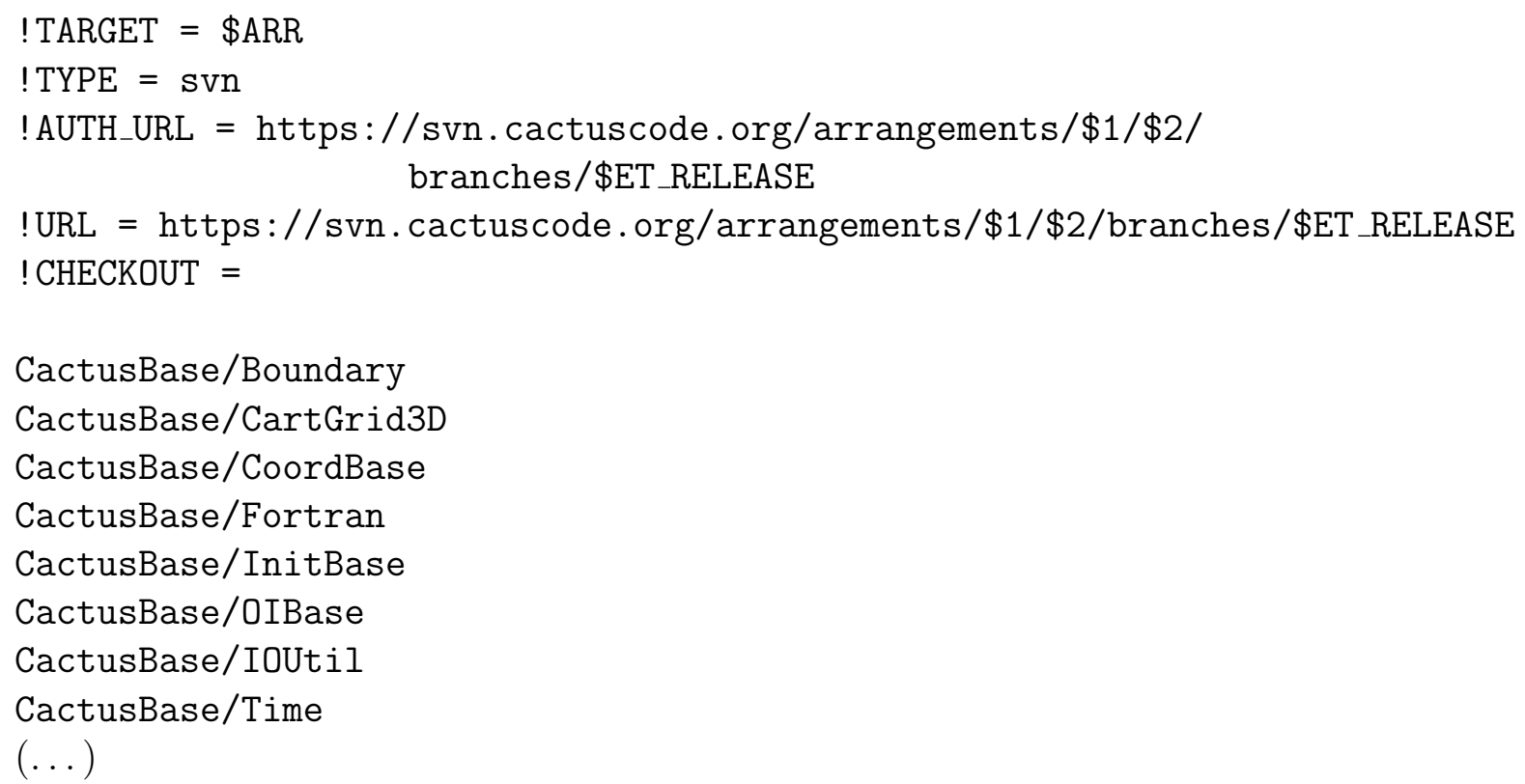

Uma vez que o GetComponents tenha sido baixado corretamente é gerada em seu ambiente de trabalho uma pasta chamada Cactus, com a seguinte estrutura:

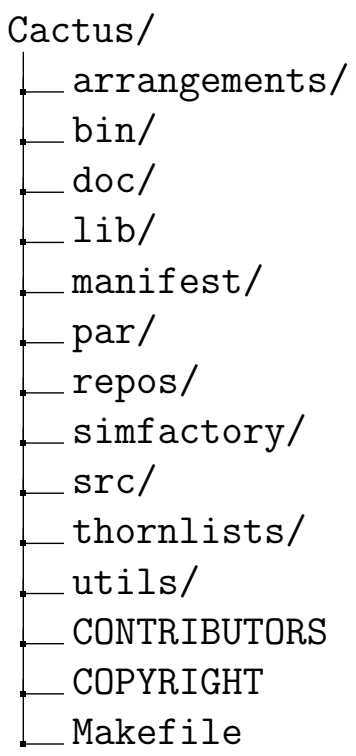

A partir desde ponto, pode-se dar continuidade ao processo de configuração e compilação. O primeiro passo é escolher o arquivo de configuração (.cfg) adequado para o sistema que estamos utilizando. Exemplos de arquivos de configuração para diferentes sistemas podem ser encontrados através do caminho de arquivos ./Cactus/simfactory/mdb/optionlists/. O segundo passo é estabelecer a thornlist apropriada para a configuração que vamos construir, dado que diferentes configurações podem coexistir compiladas desde diversas thornlist. 
É importante ressaltar que em uma simulação não são compilados todos os thorns. Por tanto, os usuários devem construir a thornlist incluindo somente os thorns necessários. A configuração pode ser feita utilizando o SimFactory. Para isso, devemos seguir as seguintes linhas de comando:

cd Cactus

./simfactory/bin/sim setup --optionlist=ubuntu.cfg--runscript debian.sh

onde o arquivo ubuntu.cfg foi escolhido de acordo ao sistema operativo utilizado pelo computador onde vai ser feita a simulação. Uma vez realizada a configuração podemos construir o executável através dos comandos

\section{./simfactory/bin/sim build [<configurationname $>]$ \\ --thornlist=einsteintoolkit.th}

Onde a expressão [configurationname] é o nome escolhido pelo usuário para melhor identificar a configuração estabelecida. Se esta é omitida da linha de comando anterior, será estabelecido por omissão para a configuração o nome "sim". Desta maneira, o sistema está preparado para fazer qualquer simulação. No presente trabalho a simulação foi feita usando uma rotina pré-definida no ET e conhecida como static_tov.par. Para a execução deste exemplo, foram utilizadas os seguintes comandos:

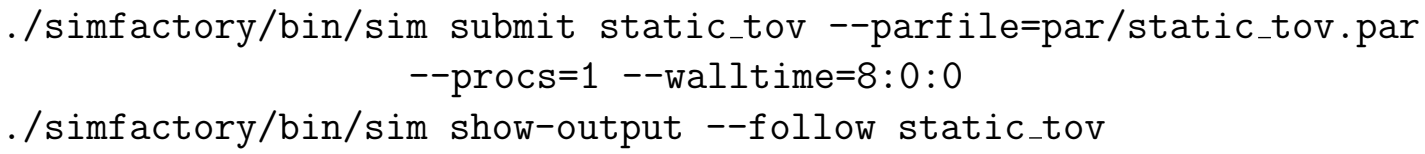

Os arquivos de exemplos fornecidos pelo próprio ET são na sua maioria muito grandes para ser compilados somente em um computador. Embora, este exemplo é mais simples e requer ao redor de $1.3 \mathrm{~GB}$ de memória RAM para compilar e será executado por cerca de 24 horas. Para aumentar a velocidade de execução, devemos reduzir a resolução mediante a mudança nos valores dos parâmetros CoordBase: :dx, CoordBase: :dy e CoordBase: :dz de 8 a 12, feito isso o tempo de compilação é reduzido a 5 horas e o consumo de memória cai a 800 MB. Logo, esta mudança pode ser feria através do comando sed da seguinte forma 
sed 'CoordBase: :d[xyz]/s/8/12' <par/static_tov.par> par/static_tov.par

O arquivo static_tov configura uma estrela TOV estática, mais especificamente uma estrela de nêutrons com uma massa de aproximadamente 1.4 massas solares, e integra o sistema combinado de equações da dinâmica de fluidos relativísticos e da evolução temporal do espaço-tempo. Adicionalmente, o espaço-tempo é evoluído através do uso da formulação BSSN 3+1 das equações de Einstein. Para verificar o status da simulação podemos usar a sequência

\section{./simfactory/bin/sim list-simulations}

Finalmente, quando a simulação é terminada (a expressão "All done"surge na tela do computador) o simfactory pode ser interrompido e é possivél analisar o output resultante, o qual se encontra no seguinte caminho de arquivos

cd $\sim /$ simulations/static_tov/output-0000/static_tov

Os outputs gerados depois de uma compilação exitosa são controlados principalmente pelos seguintes thorns: CarpetIOASCII, CarpetIOScalar, CarpetIOHDF5. O CarpetIOASCII, gera um arquivo de texto em formato ASCII contendo o output em 0, 1, 2 ou 3 dimensões das variáveis especificadas na configuração. O CarpetIOScalar é um thorn utilizado para realizar cálculos de máximo e mínimo, assim como da norma das variáveis, como o thorn anterior o output resultante é gerado como um arquivo ASCII. Finalmente, o thorn CarpetIOHDF5 tem como resultado um arquivo com extensão .h5 35].

Estes thorns utilizam, além de outros os seguintes parâmetros: out?D_vars e every?D_vars, onde ?=0,1,2, 3 é usado para representar a dimensionalidade do output, ou seja, 0-, 1-, 2-, ou 3-dimensional respectivamente. O out?D_vars está formado por uma lista de funções grid para cada uma das iterações, estabelecidas pelo parâmetro every?D_vars. Logo, o out-

put 3-dimensional é produzido ao longo de toda o grid, enquanto o output 2-dimensional é dado comumente pelos planos em 2D: xy, xz e yz, os quais contêm a origem das coorde- 
nadas. Por fim, o output em 1 dimensão é definido ao longo dos eixos coordenados x, y e z, e o output 0-D é dado por pontos específicos, geralmente é usado a origem de coordenadas.

No capítulo seguinte serão apresentados os resultados obtidos através do uso desta rotina para realizar o cálculo da densidade de energia gravitacional de uma estrela de nêutrons no formalismo da gravidade teleparalela. 


\section{Capítulo 5}

\section{Resultados e discussão}

Desde o ponto de vista dinâmico, tanto a Relatividade Geral de Einstein como a Gravidade Teleparalela fazem as mesmas predições. Embora o TERG permite a definição de quantidades de grande interesse físico, como a energia e momento angular gravitacionais, na Relatividade Geral ditas quantidades ainda estão em desenvolvimento. O objetivo deste trabalho foi calcular a densidade de energia gravitacional de uma estrela de nêutrons através de uma aproximação teleparalela em conjunto com a ferramenta numérico-computacional conhecida como Einstein Toolkit.

As estrelas de nêtrons (NS) são objetos astrofísicos extremamente compactos formados a partir do colapso gravitacional de estrelas muito massivas, com massas entre $8 M_{\odot}$ e $20 M_{\odot}$. Inicialmente, estas estrelas têm uma temperatura superior aos $10^{10} \mathrm{~K}$, que decai rapidamente devido à emissão de nêtrinos até alcançar uma temperatura de $T \sim 10^{8} \mathrm{~K}$, a qual pode ser considerada fria tendo em vista a degenerescência dos nêutrons contidos em seu interior. As massas deste tipo de estrelas estão na faixa de $M=1 \sim 3 M_{\odot}$, onde o limite superior é estabelecido pelo valor teórico conhecido como limite de Oppenheimer-Volkoff.

A existência deste tipo de estrelas, foi prevista teoricamente na década dos anos trinta [51], enquanto o primeiro modelo teórico foi desenvolvido no ano de 1939 [42, 43], onde demonstraram que as estrelas de nêutrons não poderiam ter uma massa arbitrariamente grande. Fazendo uso da Relatividade Geral eles estabeleceram o valor máximo de massa para uma estrela de nêutrons. Interessantemente este valor foi da mesma ordem de magnitude que a 


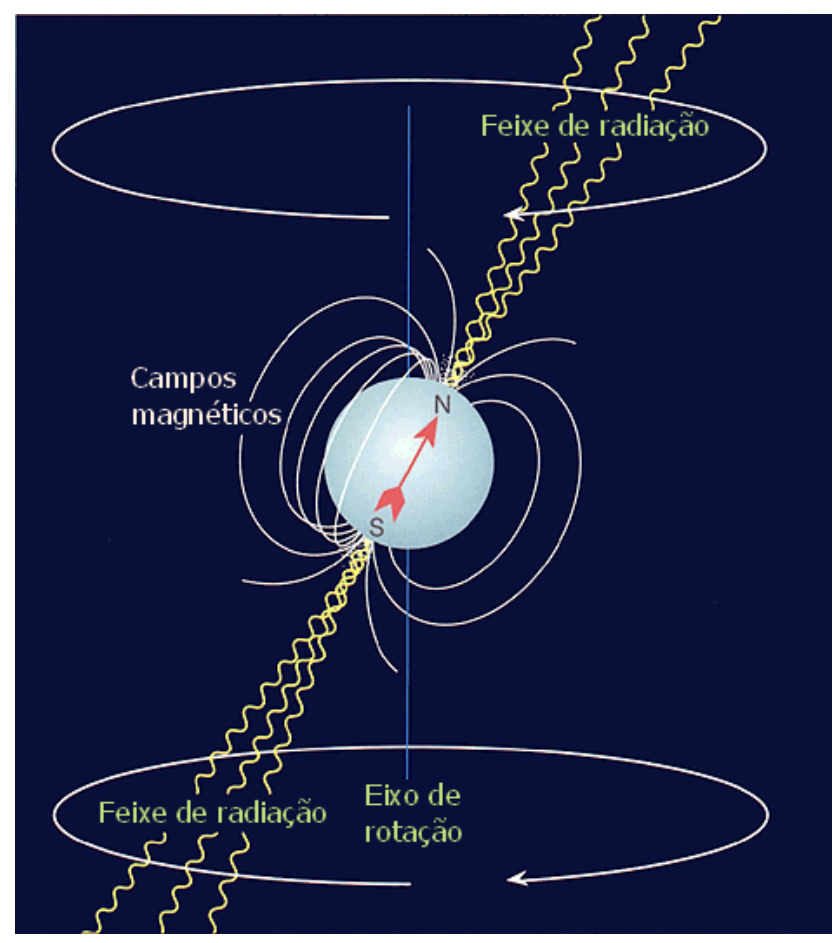

Figura 5.1: Representação de um pulsar, onde é mostrado o desalinhamento entre o eixo de rotação e os feixes de radiação emitida desde os pólos [54].

massa de Chandrasekhar, a qual determina os valores limite para a massa de uma estrela anã branca. Estudos teóricos posteriores, determinaram que as estrelas de nêutrons deveriam encontrar-se girando rapidamente e possuir campos magnéticos intensos. Pacini [52] previu que uma estrela com ditas características deveria emitir ondas de rádio. A primeira observação foi feita por radioastrônomos no ano de 1967 [53]. Eles encontraram o primeiro Pulsar, que posteriormente foi identificado como uma estrela de nêutrons em rotação.

Cerca de 2000 pulsares foram descobertos até a presente década, onde cerca de 1\%, geralmente os mais jovens, são associados com os remanescentes de uma supernova. Esta porcentagem é muita baixa devido a que o tempo de vida dos pulsares é de duas ordens de magnitude maior que o tempo de vida dos restos da supernova. Por outro lado, neste tipo de estrela o eixo magnético e o rotacional estão desalinhados. Partículas eletricamente carregadas se movem ao longo das linhas de campo magnético, fazendo com que estas escapem dos pólos emitindo ondas de rádio. Na figura (5.1) apresentamos um esquema onde é possível ver a emissão deste tipo de radiação. 
O raio típico deste tipo de estrelas está compreendido dentro do intervalo $R=9 \sim 15$ $\mathrm{km}$, definindo desta maneira a densidade média da forma

$$
\bar{\rho}=\frac{3 M}{4 \pi R^{3}}=4.8 \times 10^{14} \mathrm{~g} \mathrm{~cm}\left(\frac{M}{M_{\odot}}\right)\left(\frac{R}{10 \mathrm{~km}}\right)^{-3} .
$$

A densidade central das estrelas de nêutrons pode exceder algumas vezes o valor de $10^{15} \mathrm{~g} \mathrm{~cm}^{-3}$, sendo dito valor maior que a densidade nuclear padrão, $\rho_{0}=2.8 \times 10^{14} \mathrm{~g} \mathrm{~cm}^{-3}$, que corresponde a um número de densidade de $n_{0}=0.16$ bárions $\mathrm{fm}^{-3}\left(1 \mathrm{fm}=10^{-13} \mathrm{~cm}\right)$. Logo, neste tipo de estrelas, o mecanismo que prevê o colapso gravitacional é a pressão de degenerescência predominantemente dos nêutrons e de algumas partículas exóticas como píons, kaons ou quarks [55]. A luminosidade proveniente da superfície da estrela é escrita em termos da temperatura $T$ e o raio $R$ da forma

$$
L=4 \pi R^{2} \sigma T^{4}=7 \times 10^{32} \operatorname{erg~s}^{-1}\left(\frac{R}{10 \mathrm{~km}}\right)^{2}\left(\frac{T}{10^{6} \mathrm{~K}}\right)
$$

e onde $\sigma$ é a constante de Stefan-Boltzmann. Para valores de $R$ muito pequenos temos também uma área superficial muito pequena, que em conjunto com temperaturas superficiais de até $10^{6}$ não geram uma luminosidade o suficientemente forte para permitir a detecção das estrelas de nêutrons ao longo da galaxia. Na verdade, estas estrelas são comumente descobertas através da detecção das ondas de rádio que emitem ditas estrelas em rotação. Logo, este tipo de estrelas, têm uma luminosidade igual a

$$
L=-I \Omega \dot{\Omega}
$$

onde $I \sim M R^{2} \sim 10^{45} \mathrm{~g} \mathrm{~cm}^{2}$ é o momento de inércia, $\Omega$ é a frequência angular e $\dot{\Omega}$ é sua derivada temporal. Um dos pulsares mais conhecidos, descoberto na Nebulosa do Caranguejo pouco tempo depois da primeira observação de pulsares, é o pulsar do Caranguejo, que tem um período de $P=2 \pi / \Omega=33 \mathrm{~ms}$ e com taxa de variação no tempo de $\dot{P} \simeq 4 \times 10^{-13}$, gerando desta maneira uma luminosidade de $L \sim 10^{38} \mathrm{erg} \mathrm{s}^{-1}$, a qual é 5 ordens de magnitude maior que a luminosidade solar. O pulsar do Caranguejo irradia em todas as bandas do espectro electromagnético, portanto a energia que libera como ondas de rádio representa somente uma pequena fração desta enorme fonte de energia [55]. Logo, rádio pulsares são 
concebidos como estrelas de nêutrons altamente magnetizadas que giram rapidamente, onde os pulsos são consequência do chamado "efeito lighthouse" que faz com que a radiação seja vista como intermitente.

Estrelas de nêutrons também foram descobertas como pulsares que emitem raios X [56], formam um sistema binário com outro tipo de estrela, geralmente mais jovem. Estes pulsares são objetos gravitacionalmente fortes que acretam matéria desde sua estrela companheira, portanto a luminosidade da radiação é devida ao processo de acreção sobre a estrela de nêutrons e pode ser calculada da seguinte forma

$$
L=\frac{G M \dot{M}}{R}
$$

onde $\dot{M}$ é a taxa de acreção sobre a NS. A radiação emitida desde a coluna de acreção ou desde o hotspot na superfície é controlada pela taxa de ratoção da estrela. Nos casos onde a massa da estrela companheira é baixa, a transferência de matéria é feita através do lóbulo de Roche desde a estrela de baixa massa até a magnetosfera da NS. Por outro lado, se a estrela que acompanha a NS está perdendo grandes quantidades de massa devido ao vento estelar, o processo de acreção desde a NS pode ser feito sobre este vento. Um tipo especial destes sistemas binários, é aquele conformado por uma NS e uma estrela Be massiva, onde a estrela de nêutrons tem uma órbita excêntrica ao redor da estrela Be, caracterizada por um disco circunstelar formado por material ejetado da estrela.

Desde meados dos anos 90 do século passado, foram identificadas algumas outras famílias de NS, tais como Pulsares Anômalos de raios $x$, Repetidores de raios Gamma e Pulsares Isolados. Na atualidade os Repetidores e os Pulsares Anômalos são comumente utilizados para representar Magnetares, os quais são estrelas de nêutrons fortemente magnetizadas cujo campo magnético decai no tempo.

O campo magnético típico de uma estrela de nêutros em rotação é de $\sim 10^{12} \mathrm{G}$. A origem desde forte campo magnético é ainda desconhecida, mas uma das teorias mais aceitas até a presente época, é a teoria da conservação do fluxo magnético. Desta maneira, se a estrela massiva progenitora começa o processo de colapso gravitacional com uma área superficial 
muito maior que a estrela resultante, a teoria da conservação do fluxo de energia estabelece que o campo magnético na estrela de nêutrons deve ser muito forte para assim garantir dita conservação. Estrelas de nêutrons em sistemas binários de baixa massa e emissoras de raios $\mathrm{X}$, têm campos magnéticos muito baixos, $B \sim 10^{9} \mathrm{G}$, possivelmente como uma consequência dos processos de acreção relacionados a ela. Adicionalmente, o campo magnético dos Magnetares é de aproximadamente $B \sim 10^{15} \mathrm{G}$ excedendo em duas ordens de magnitude o limite crítico quântico $B_{c} \equiv m_{e}^{2} c^{3} / e \hbar=4.4 \times 10^{13} \mathrm{G}$, onde a energia de ciclotron dos elétrons é igual a sua energia de massa em repouso. Estes campos magnéticos intensos influenciam a estrutura dos átomos além de estimular o início de alguns processos da eletrodinâmica quântica, como a polarização e a birrefringência. Se espera que o campo magnético interno dos magnetares seja incluso muito maior. Porém existe um limite superior para o valor dos campos internos, o qual é determinado pelo equilíbrio da energia de ligação $\sim G M^{2} / R$ e a energia magnética $\sim \int\left(B^{2} / 8 \pi\right) d V$, resultando no valor aproximado de $B_{\max } \sim 10^{18}$.

No presente trabalho foi utilizada a rotina static_tov.par pré-definida do ET, a qual implementa o thorn TOVSolver para resolver o sistema de equações TOV para a pressão, a massa gravitacional $M_{e}$ e o potencial gravitacional $\Phi=\log \alpha$ no interior de uma estrela esfericamente simétrica e em equilíbrio hidrostático. O sistema estabelecido pelo TOVSolver é evoluído usando conjuntamente o método BSSN, implementado no McLanchlan, com o sistema de evolução hidrodinâmica, implementado no GRHydro. Enquanto que a integração temporal das equações diferenciais parciais foi realizada pelo thorn MoL, que implementa o método Runge-Kutta de $4^{a}$ ordem.

Na simulação apresentada neste trabalho foi estabelecida uma estrela TOV estável, descrita pela equação de estado politrópica, $p=K \rho_{c}^{\Gamma}$, expressa em termos da pressão $p$ e a densidade de energia central $\rho_{c}$. Apesar de que a estrela em consideração tem simetria esférica, a rotina static_tov.par utiliza para descrever o interior da mesma a métrica escrita em coordenadas cartesianas da forma

$$
d s^{2}=g_{t t} d t^{2}+g_{x x} d x^{2}+g_{y y} d y^{2}+g_{z z} d z^{2}
$$

Adicionalmente, esta rotina considera que a massa do interior da estrela esta constituída 
por um fluido perfeito com tensor de energia-momento igual a

$$
T^{\mu \nu}=(\epsilon+P) u^{\mu} u^{\nu}+P g^{\mu \nu}
$$

escrito em termos da densidade de energia total $\epsilon$, a pressão $P$ e a quadrivelocidade $u^{\mu}$. Desta maneira, este modelo pode ser utilizado para representar uma NS não rotante com massa de $M=1.4 M_{\odot}$. Logo, para uma NS constituída principalmente por nêutrons não relativísticos, temos que o índice adiabático é igual a $\Gamma=2$, a constante $K$ é igual a 100 e a densidade central inicial está definida como $\rho_{c}=1.28 \times 10^{-3}[57]$. Por outro lado, nesta rotina os valores iniciais das variáveis ADMBase foram determinados pelo thorn TOVSolver, ao mesmo tempo que os dados iniciais para as derivadas temporais das funções de calibre foram estabelecidos como zero.

Para resultados precisos e significativos da evolução do sistema, a simulação foi realizada usando 5 níveis de refinamento sob um grid cúbico definido no arquivo static_tov ·par pelas seguintes linhas de comando

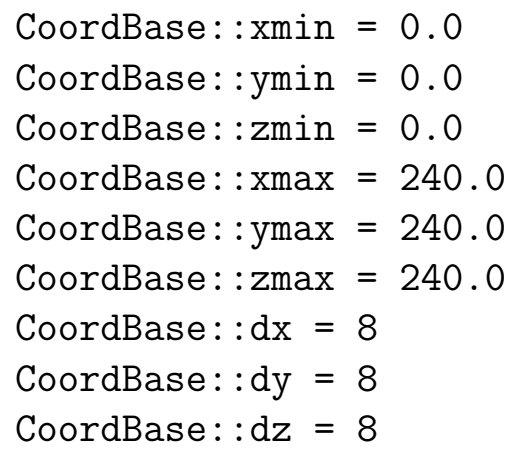

onde os pontos (xmin, ymin, zmim) e (xmax, ymax, zmax) estabelecem tanto o limite inferior, como o limite superior do domínio físico considerado na simulação. Os parâmetros CoordBase : $: \mathrm{dx}=8$, CoordBase $: \mathrm{dy}=8$ e CoordBase $: \mathrm{dz}=8$, representam o espaçamento inicial das células do grid nas direções x, y e z, respectivamente. Para cada nível de refinamento a resolução é duplicada. Portanto, o espaçamento entre as células do grid cai à metade, gerando desta maneira uma variação no tamanho da região a refinar. Logo, em nossa simulação foram utilizados adicionalmente 4 grids com tamanhos de 120M, 60M, 30M e 15M, para refinar o espaço ao redor da estrela centrada na origem. A resolução típica destes novos grids 
vai desde $0.125 \mathrm{M}$ até $2 \mathrm{M}$, onde para $1 \mathrm{M}$ vamos ter somente algumas dezenas de pontos ao londo do diâmetro da estrela, por conseguinte esta é uma das menores resoluções usadas na simulação que ainda produz bons resultados. Os novos tamanhos dos grids são estabelecidos pelo thorn CarpetRegrid2 da forma

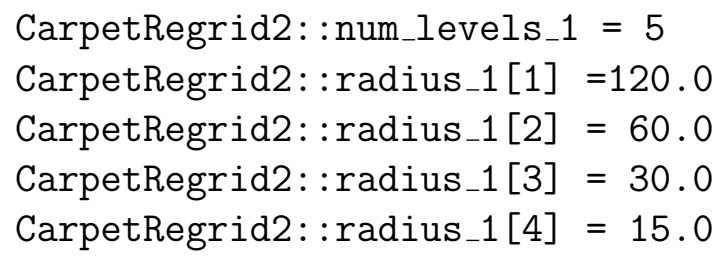

onde a primeira linha de comandos estabelece os níveis de refinamento a aplicar na simulação, e as outras linhas estabelecem o tamanho dos grids adicionais. As dimensões ou tamanho das células do grid estarão fortemente associados com o nível de refinamento da análise do sistema astrofísico em questão. Por outro lado, enquanto mais células tenha o grid, mais recursos computacionais serão necessários para a simulação númerica. Em nossa simulação o tempo de compilação foi reduzido consideravelmente devido ao uso do thorn ReflectionSymmetry, que fornece simetria ao problema estudado.

Quando a simulação foi finalizada com sucesso, foi possível visualizar e posteriormente analisar o output, que como já foi dito no capítulo anterior é gerado em diferentes arquivos e com diferentes formatos. Por exemplo, o arquivo ADMBase: :metric.x.asc contém a seguinte informação:

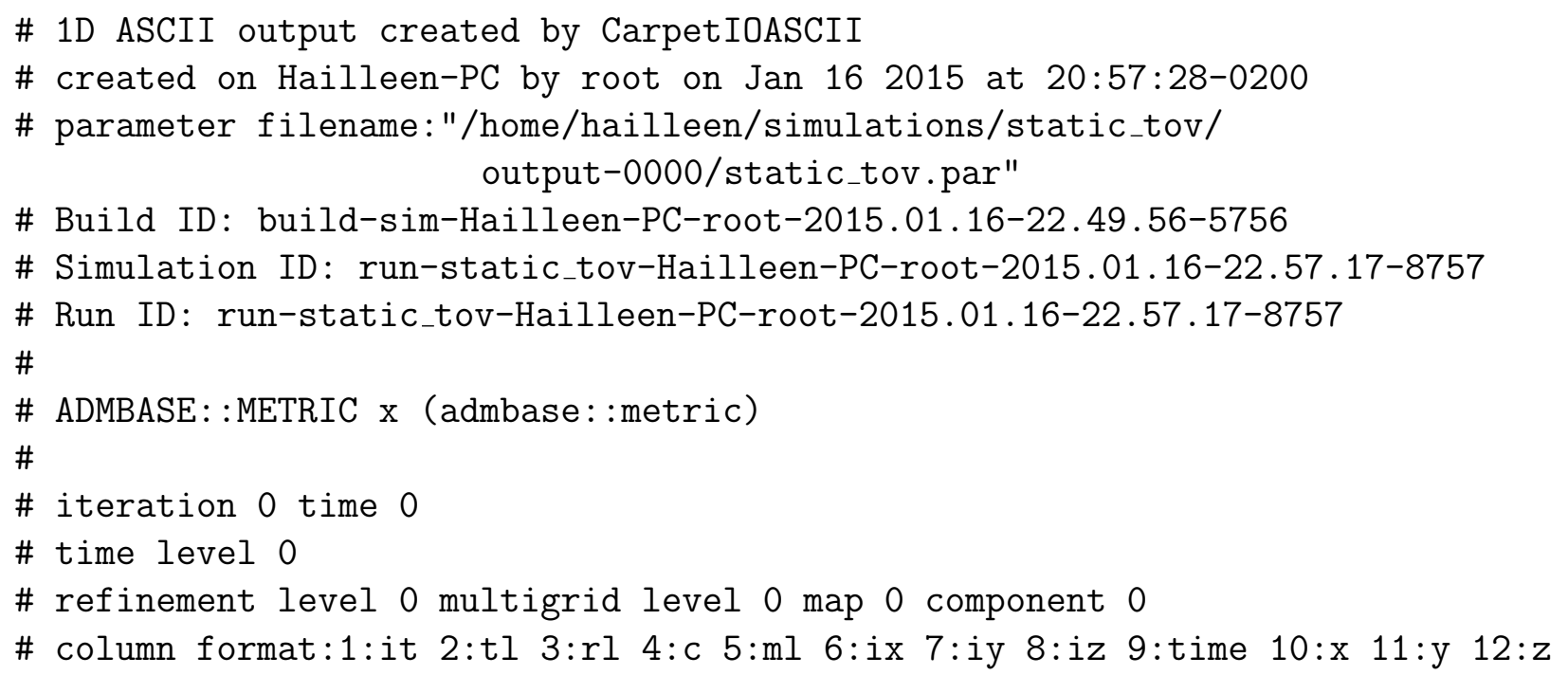




\section{\# data columns: 13:gxx 14:gxy 15:gxz 16:gyy 17:gyz 18:gzz}

Nas primeiras linhas é especificado o tipo de arquivo, o computador onde foi realizada a simulação, a data e a hora, assim como a rotina que foi compilada. Este arquivo contém os dados da variação da métrica ao longo do eixo x, enquanto as outras duas coordenadas, $\mathrm{y}$ e z, são fixadas em zero. Depois são apresentados em forma de tabela os dados gerados em dita simulação. O parâmetro time é definido na rotina static_tov.par usando a linha de comando Cactus: $:$ cctk_final_time $=1000$, onde dita quantidade representa o número de iterações a serem feitas para os diferentes valores do nível de refinamento. Na figura (5.2) é mostrada de forma parcial a tabela de dados, onde as primeiras cinco colunas da esquerda para a direita representam respectivamente a iteração (it), o nível de tempo (tl), nível de refinamento (rl), a componente (c) e o nível de multigrid (ml). Logo, temos as iterações para as coordenadas x,y e z (ix, iy, iz), o tempo (time), as coordenadas x, y, z. Os parâmetros tl, ml e c são fixados em zero ao longo da simulação. Logo, os valores de $\mathrm{x}$ dependem do nível de refinamento ( $r l)$ que esta sendo aplicado ao grid. O parâmetro ix, que representa o número da iteração feita na coordenada $\mathrm{x}$, também depende do valor de rl. Finalmente, temos que as últimas 6 colunas representam os valores das componentes da métrica quando variamos a coordenada x. Dado que os coordenadas são ortogonais entre si, a métrica é diagonal e portanto as componentes cruzadas são nulas.

Por outro lado, para realizar o cálculo da energia gravitacional de uma NS no formalismo do TEGR, primeiro devemos estabelecer nosso espaço-tempo, o qual está definido para um observador estacionário. Portanto o campo de tétradas vem escrito em termos da métrica em coordenadas cartesianas da forma

$$
e_{\mu}^{a}=\left(\begin{array}{cccc}
\sqrt{-g_{t t}} & 0 & 0 & 0 \\
0 & \sqrt{g_{x x}} & 0 & 0 \\
0 & 0 & \sqrt{g_{y y}} & 0 \\
0 & 0 & 0 & \sqrt{g_{z z}}
\end{array}\right)
$$

Logo, ao combinar o campo de tétrada (5.7) e o tensor $\Sigma^{a b c}$ definido na equação (3.31), a equação para a energia gravitacional no formalismo teleparalelo, expressão (3.58), pode ser 


\begin{tabular}{|c|c|c|c|c|c|c|}
\hline 0 & 0 & 0 & 00 & 2048 & 1536 & 1536 \\
\hline 0 & 0 & 0 & 00 & 2560 & 1536 & 1536 \\
\hline 0 & 0 & 0 & 00 & 3072 & 1536 & 1536 \\
\hline 0 & 0 & 0 & 00 & 3584 & 1536 & 1536 \\
\hline 0 & 0 & 0 & 00 & 4096 & 1536 & 1536 \\
\hline 0 & 0 & 0 & 00 & 4608 & 1536 & 1536 \\
\hline 0 & 0 & 0 & 00 & 5120 & 1536 & 1536 \\
\hline 0 & 0 & 0 & 00 & 5632 & 1536 & 1536 \\
\hline 0 & 0 & 0 & 00 & 6144 & 1536 & 1536 \\
\hline 0 & 0 & 0 & 00 & 6656 & 1536 & 1536 \\
\hline 0 & 0 & 0 & 00 & 7168 & 1536 & 1536 \\
\hline 0 & 0 & 0 & 00 & 7680 & 1536 & 1536 \\
\hline 0 & 0 & 0 & 00 & 8192 & 1536 & 1536 \\
\hline 0 & 0 & 0 & 00 & 8704 & 1536 & 1536 \\
\hline 0 & 0 & 0 & 00 & 9216 & 1536 & 1536 \\
\hline 0 & 0 & 0 & 00 & 9728 & 1536 & 1536 \\
\hline 0 & 0 & 0 & 00 & 10240 & 1536 & 1536 \\
\hline 0 & 0 & 0 & 00 & 10752 & 2 1536 & 1536 \\
\hline
\end{tabular}

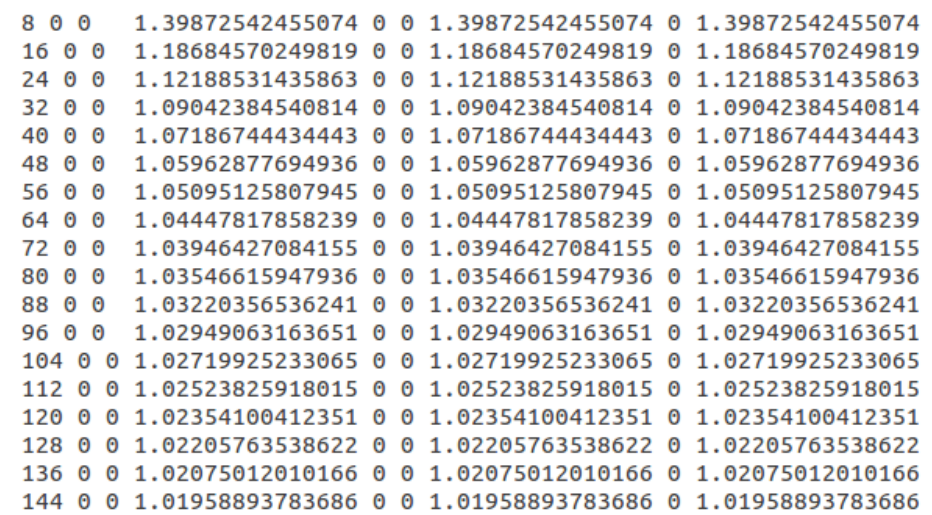

Figura 5.2: Vista parcial da tabela de dados gerados no arquivo ADMBase: :metric.x.asc produto da simulação da rotina static_tov.par. As primeiras cinco colunas da esquerda para a direita representam respectivamente a iteração (it), o nível de tempo (tl), nível de refinamento ( $\mathrm{rl}$ ), a componente (c) e o nível de multigrid (ml). Logo, temos as iterações para as coordenadas x,y e z (ix, iy, iz), o tempo (tempo), as coordenadas $\mathrm{x}, \mathrm{y}, \mathrm{z}$ e finalmente os valores das componentes da métrica quando variamos a coordenada $\mathrm{x}$.

escrita como

$$
E=4 k \oint_{S_{i}} e \Sigma^{(0) 0 i} d S_{i}
$$

onde $d S_{i}$ é o diferencial de área na direção $i$ e $k=1 / 16 \pi$. Dado que nossa simulação foi feita sob um grid cúbico de tamanho finito, o diferencial de integração da equação anterior pode ser transformado em uma hipersuperfície cúbica de dimensões $x_{0}$, $y_{0}$ e $z_{0}$, obtendo-se desta maneira a energia gravitacional de uma estrela de nêutrons

$$
E=4 k\left[\lim _{x \rightarrow x_{0}} \int e \Sigma^{(0) 01} d S_{1}+\lim _{y \rightarrow y_{0}} \int e \Sigma^{(0) 02} d S_{2}+\lim _{z \rightarrow z_{0}} \int e \Sigma^{(0) 03} d S_{3}\right]
$$

As componentes do tensor $\Sigma^{(0) 0 i}$ são expressões exatas na simetria esférica, portanto no limite em que a hipersuperfície é muito grande, ditas tendem a

$$
\begin{aligned}
\Sigma^{(0) 01} & =-\frac{1}{4}\left[\frac{\partial_{x}\left(g_{y y} g_{z z}\right)}{\sqrt{-g_{t t}} g_{x x} g_{y y} g_{z z}}\right], \\
\Sigma^{(0) 02} & =-\frac{1}{4}\left[\frac{\partial_{y}\left(g_{x x} g_{z z}\right)}{\sqrt{-g_{t t}} g_{x x} g_{y y} g_{z z}}\right], \\
\Sigma^{(0) 03} & =-\frac{1}{4}\left[\frac{\partial_{z}\left(g_{x x} g_{y y}\right)}{\sqrt{-g_{t t}} g_{x x} g_{y y} g_{z z}}\right] .
\end{aligned}
$$

Tendo em vista que $e=\sqrt{-g}$ onde $g=g_{t t} g_{x x} g_{y y} g_{z z}$ é o determinante do tensor métrico, e devido ao fato que a equação (5.9) pode ser reescrita usando o teorema da divergência, temos 
o seguinte

$$
E=-k \lim _{V \rightarrow V_{0}} \int\left\{\partial_{x}\left[\frac{\partial_{x}\left(g_{y y} g_{z z}\right)}{\sqrt{g_{x x} g_{y y} g_{z z}}}\right]+\partial_{y}\left[\frac{\partial_{y}\left(g_{x x} g_{z z}\right)}{\sqrt{g_{x x} g_{y y} g_{z z}}}\right]+\partial_{z}\left[\frac{\partial_{z}\left(g_{x x} g_{y y}\right)}{\sqrt{g_{x x} g_{y y} g_{z z}}}\right]\right\} d x^{3},
$$

onde o volume $V_{0}$ é definido como $V_{0}=x_{0} y_{0} z_{0}$, e com a quantidade entre chaves conhecida como Densidade de energia gravitacional $\epsilon_{g}$. A expressão (5.13) pode ser calculada usando os dados gerados pelo ET sempre que seja conhecida a variação das componentes da métrica em todo o espaço. Logo, devido que para gerar todos aqueles dados se requer de muito tempo de simulação, o ET somente calcula a variação das componentes da métrica na origem do espaço. Desta maneira nosso trabalho foi calcular a densidade de energia gravitacional de uma NS na origem. Ao definir as quantidades $\gamma=g_{x x} g_{y y} g_{z z}$ e $\delta=\sqrt{\gamma}$,e considerando que a métrica é diagonal, ou seja, $g_{i i}=1 / g^{i i}$, a densidade de energia gravitacional pode ser reescrita como

$$
\begin{aligned}
E & =-k \lim _{V \rightarrow V_{0}} \int\left[\partial_{i} \partial^{i} \delta+\partial_{i} \partial_{j}\left(\delta g^{i j}\right)\right] d x^{3}, \\
& =-k \lim _{V \rightarrow V_{0}} \int \epsilon_{g} d x^{3} .
\end{aligned}
$$

onde $\epsilon_{g}=\partial_{i} \partial^{i} \delta+\partial_{i} \partial_{j}\left(\delta g^{i j}\right)$. O cálculo da densidade de energia gravitacional foi realizado através de um código simples em linguagem python, onde as derivadas parciais foram resolvidas numericamente utilizando o método de Diferenças Finitas, mais especificamente o método central com precisão de $8^{a}$ ordem. Logo, na figura (5.3) é apresentada a variação da densidade de energia $\epsilon_{g}$ ao longo de cada um dos eixos coordenados. Esta densidade foi calculada para cada um dos eixos fazendo uso dos arquivos ADMBase: :metric.x.asc, ADMBase: :metric.y.asc e ADMBase::metric.z.asc, respectivamente. A semelhança entre os gráficos é devida à simetria do problema. Consideremos a figura (5.3a), nesta imagem é possível observar como para pontos com coordenada $x$ entre 0 e $\sim 10 \mathrm{M}$ a densidade de energia se mantém negativa até chegar a zero. Portanto é possível concluir que dita densidade de energia é utilizada no interior da estrela para evitar o colapso gravitacional, já que ela cria uma pressão o suficientemente grande que compensa a força de gravidade garantindo desta maneira o equilíbrio hidrostâtico da estrela. Por outro lado, para pontos com coordenada $x \gtrsim 10$ M temos uma densidade de energia positiva que cai rapidamente a zero na medida que estamos mais longe da estrela. Logo, dita densidade de energia poderia relaciona-se com a 
força gravitacional que a estrela exerce sobre os outros corpos do espaço, deste modo quanto maior a distância da estrela de nêutrons, menor vai ser sua força gravitacional.

Finalmente, usando o arquivo de dados hydrobase: :rho.maximum.asc, gerado na simulação, é possível mostrar a evolução no tempo da densidade central da estrela (figura (5.4)). Para os primeiros estágios da evolução temos uma clara diminuição no valor da densidade máxima, onde este pico pode ser interpretado como o resultado da interpolação da solução unidimensional de equilíbrio sobre a solução tridimensional da evolução. Logo, o valor máximo da densidade oscila ao longo do tempo, evidenciando uma tendência ascendente e com uma diminuição da amplitude em torno ao valor inicial. Mesmo que o modelo tenha sido considerado em equilíbrio, os erros númericos sugerem que a estrela não está exatamente neste estado, portanto a estrela oscila. Logo, a energia de oscilação é dissipada lentamente, diminuindo a amplitude de oscilação enquanto a estrela contrai seu volume, incrementando desta maneira sua densidade central máxima. 


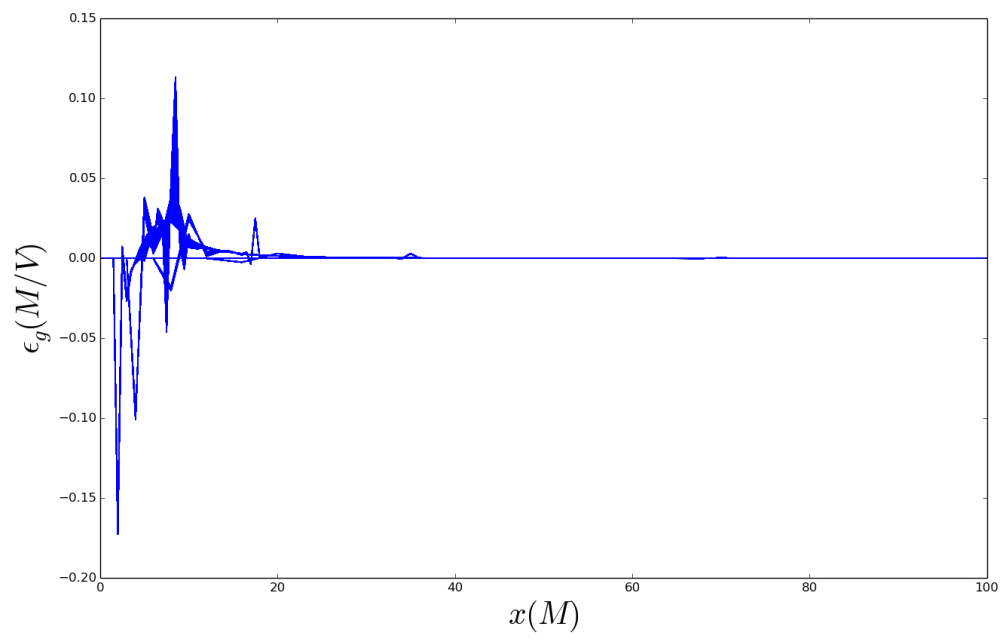

(a) $\operatorname{Eixo} x$

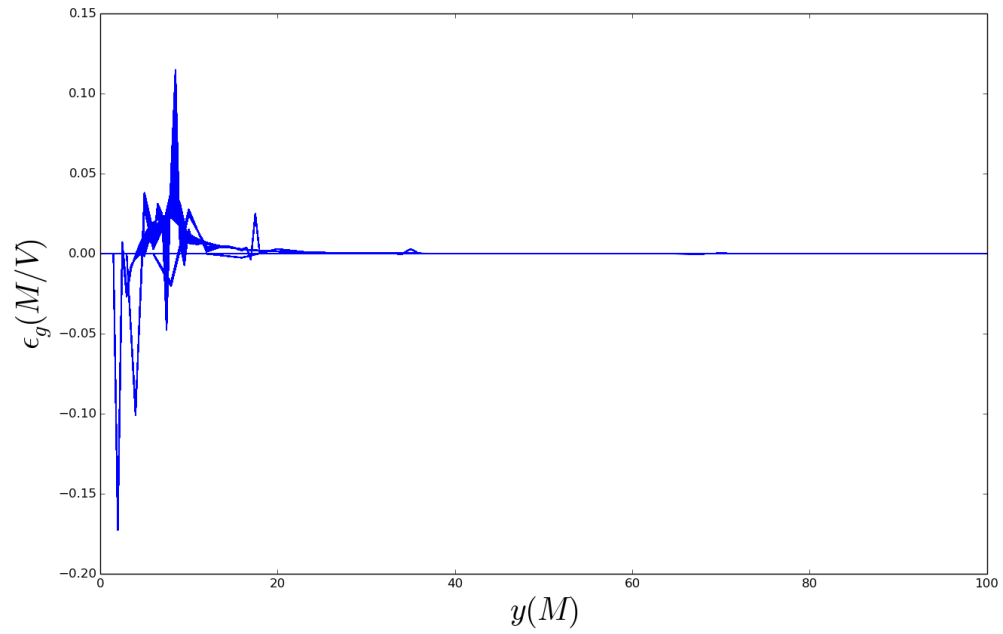

(b) Eixo $y$

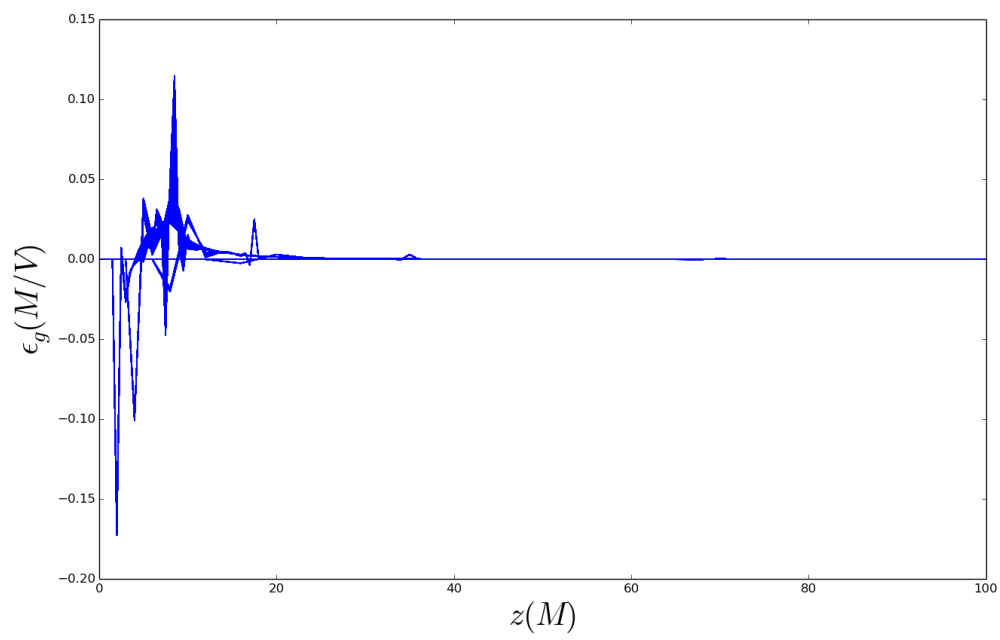

(c) Eixo $z$

Figura 5.3: Variação da densidade de energia gravitacional $\epsilon_{g}$ ao longo dos eixos (a) $x$, (b) y y (c) z. Para valores das coordenadas menores que $\sim 10 \mathrm{M}$, temos densidade de energia negativa, que pode ser responsável pelo equilíbrio hidrostático da estrela. Para pontos com coordenada maior que $\sim 10 \mathrm{M}$ se observa um rápido decaimento da densidade de energia gravitacional. 


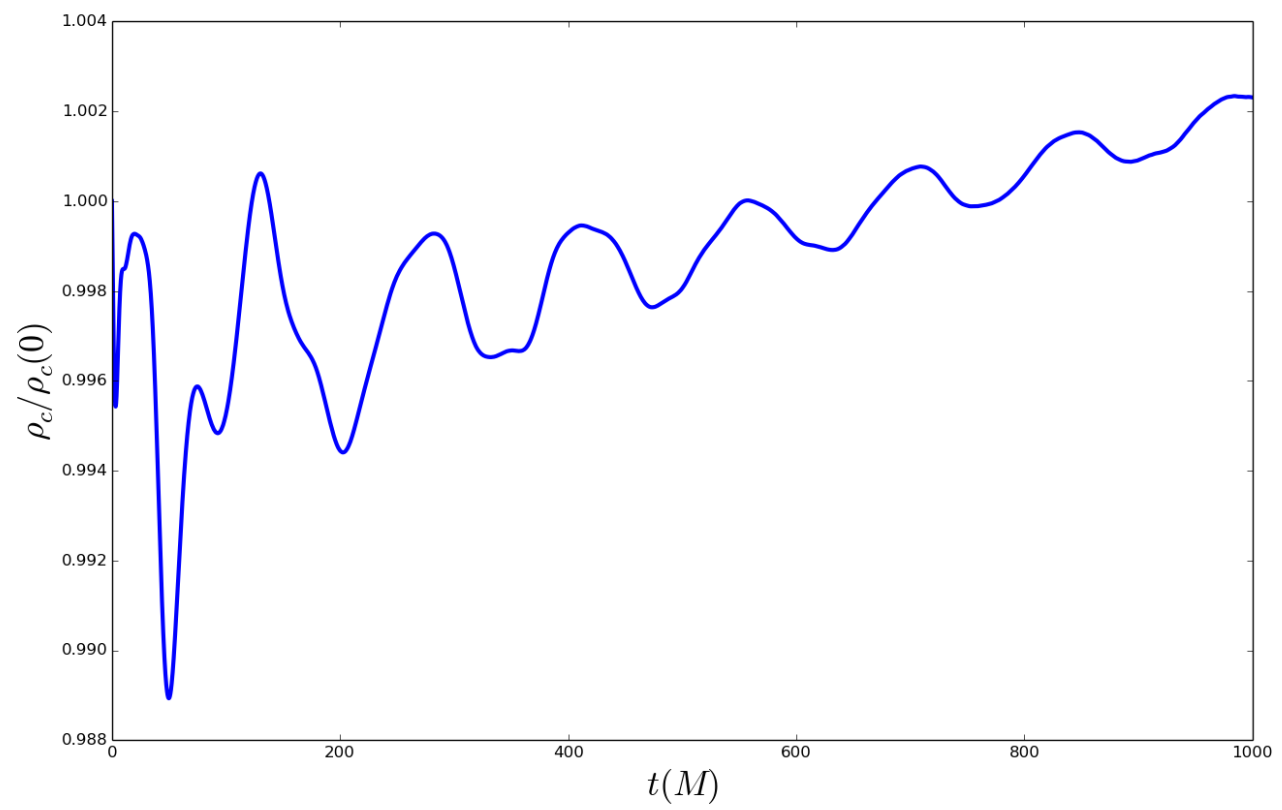

Figura 5.4: Evolução da densidade central de uma estrela TOV ao longo do tempo. Inicialmente temos um pico produzido pela interpolação da solução de equilíbrio na evolução do grid. A evolução da densidade central é dominada pela interação da superfície da estrela com a atmosfera artificial [35]. 


\section{Capítulo 6}

\section{Conclusões}

No presente trabalho analisamos a física de uma estrela de nêutrons desde o ponto de vista da Gravidade Telaparalela e em conjunto com a ferramenta numérico-computacional conhecida como Einstein Toolkit. Partindo do formalismo lagrangiano do Teleparalelismo Equivalente à Relatividade Geral, foi possível escrever uma expressão para a densidade de energia gravitacional em termos da métrica $g_{\mu \nu}$ do espaço-tempo em consideração. Esta métrica foi calculada usando a rotina static_tov.par pré-definida no ET, que resolve as equações TOV no interior de uma estrela de nêutrons completamente simétrica, estática e em equilíbrio hidrostático. Na figura (5.3) mostramos a variação da densidade de energia gravitacional ao longo dos eixos coordenados. Para pontos como coordenada menor que 10M podemos ver como a densidade gravitacional tem valores negativos até alcançar o zero. Nesta região é possível concluir que dita densidade de energia contribui com o equilíbrio hidrotático da estrela, criando uma pressão o suficientemente grande para compensar a forte força gravitacional e assim evitar seu colapso. Por outro lado, para pontos com coordenadas maiores que $\sim 10 \mathrm{M}$ se evidencia uma densidade de energia positiva que cai rapidamente a zero na medida que estamos mais longe do centro da estrela. Desta maneira dita densidade de energia poderia se relacionar com a força gravitacional que a estrela exerce sobre outros

corpos celestes do espaço. É importante destacar que devido à oscilação dos dados, os erros nos resultados são consideravelmente grandes.

Adicionalmente, fazendo uso de dados resultantes da simulação também foi possível mostrar a evolução temporal da densidade central da estrela. Na figura 5.4, se mostra que 
ao longo do tempo a densidade central oscila entorno a um valor máximo, evidenciando-se uma tendência ascendente em conjunto com uma diminuição da amplitude de oscilação. Este comportamento poderia dever-se que a estrela não se encontra completamente em equilíbrio, em lugar disso ela está oscilando. Portanto, na medida em que a energia de oscilação é dissipada, a estrela reage contraindo seu volume e desta maneira aumenta sua densidade central.

O presente trabalho mostra como resultados dois aspectos importantes: a importância do cálculo da energia gravitacional de um corpo celeste, o qual não é possível ser feito no formalismo da Relatividade Geral, além da implementação de uma ferramenta computacional criada especialmente para auxiliar na resolução de fenômenos astrofísicos como sistemas binários de buracos negros e estrelas de nêutrons, formação de ondas gravitacionais, sistemas isolados de estrelas de nêtrons e uma gama de fenômenos relativísticos. Para trabalhos subsequentes poderia considera-se a modificação das condições iniciais do sistema, tratamento dos campos magnéticos, equações de estado muito mais complexas, estudo das reações nucleares, além da implementação de thorns e rotinas de autoria própria. 


\section{Referências Bibliográficas}

[1] A. Einstein, Prinzipielles Zur Allgemeinen Relativitätstheorie. Annalen der Physik. 55, 1918.

[2] A. Einstein, Auf die Riemann-Metrik und den Fern-Parallelismus gegründete einheitliche Feldtheorie. Mathematische Annalen. 102, 1930.

[3] J. W. Maluf, J. F. da Rocha-Neto, T. M. L. Totibio, K. H. Castello-Branco, Energy and Angular Momentum od the Gravitational Field in the Teleparallel Geometry. Physical Review. D65, 124001. 2002.

[4] Einstein Toolkit: Open Software for Relativistic Astrophysic, URL http://einsteintoolkit.org/

[5] H.M. Nussenzveig, Curso de Física Básica Vol 4. Ótica, Relatividade e Física Quântica. Editora Blucher, $2^{a}$ Edição, 2014.

[6] R. d'Inverno, Introducing Einstein's Relativity, $3^{\text {ra }}$ Edição. Cambridge University Press. Cambridge, 2004.

[7] B. Schutz, A First Course in General Relativity. $2^{d a}$ Edição. Cambridge University Press. Cambridge, 2009.

[8] S. Carroll, Spacetime and Geometry. An Introduction to General Relativity. Addison Wesley, 2003.

[9] R. Feynman, F. Morinigo, W. Wagner, Feynman Lectures on Gravitation. $1^{\text {ra }}$ Edição. Westview Press, 2002.

[10] C. Herdeiro, Notas de Teoria da Relatividade. Volumen 1, Departamento de Física, Universidade de Aveiro. Portugal, 2011. 
[11] G. t'Hooft. Introduction to General Relatitity. Institute for Theoretical Physics, Utrecht University. Holanda, 2010.

[12] R. Weitzenböck, Invariantem Theorie. Nordhoff, Groningen, 1923.

[13] C. Møller, Conservation Laws and Absolute Paralleslism in General Relativity. K. Dan. Vidensk, Selsk. Mat. Fys. Skr. 1, 10, 1961.

[14] C. Pellegrini, J. Plebanski, Tetrad Fields and Gravitational Fields K. Dan. Vidensk, Selsk. Mat. Fys. Skr. 2, 4, 1963.

[15] Y. M. Cho, Einstein Lagrangian as the Translational Yang-Mills Lagrangian. Physical Review. D14, 2521, 1976.

[16] K. Hayashi, T. Shirafuji, New General Relativity. Physical Review. D19, 3524, 1979.

[17] F. W. Hehl, Four lectures on Poincaré Gauge Field Theory, in Cosmology and Gravitation: Spin, Torsion, Rotation ans Supergravity. Editado por P.G. Bergmann e V. de Sabbata, Plenum Press. New York, 1980.

[18] J. Nitsch, The Macroscopic Limit of the Poincaré Gauge Field Theory on Gravitation, in Cosmology and Gravitation: Spin, Torsion, Rotation ans Supergravity. Editado por P.G. Bergmann e V. de Sabbata, Plenum Press. New York, 1980.

[19] J. W. Maluf. The Teleparallel equivalente to General Relativity. Annanlen der Physik, Vol 525, 339, 2013.

[20] S. C. Ulhoa, O Teleparalelismo Equivalente à Relatividade Geral e o Momento Angular Gravitacional. Physiæ 8, 11, 2009.

[21] F. W. Hehl, Proceedings of the 6th School of Cosmology and Gravitation on Spin, torsion, Rotation an Supergravity. Editado por P.G. Bergmann V. de Sabbata. Plenum, New York, 1980; F. W. Hehl, J. D. MacCrea, E. W. Mielke and Y. Ne'eman, Phys. Rep. 258, $1,1995$.

[22] Y. N. Obukov e J. G. Pereira, Metric-affine Approach to Teleparallel Gravity. Physical Review. D67, 044016, 2003. 
[23] M. Blagojevic e M. Vasilic, Conservation Laws in the Teleparallel Theory of Gravity.Physical Review. D64, 044010, 2001.

[24] C. Møller, Conservation Laws in the Tetrad Theory of Gravitation. Proceedings of the conference on Theory of Gravitation, Warszawa and Jablona 1962 (PWN- Polish Scientific Publishers, Warszawa and Gauthier-Villars, Paris, 1964).

[25] J. M. Nester, Positive Energy via the Teleparallel Hamiltonian.International Journal of Modern Physics A. 4, 1755, 1989.

[26] V. C. de Andrade e J. G. Pereira, Gravitational Lorentz Force and the Description of the Gravitational Interaction. Physical Review. D56, 4689, 1997.

[27] J.W. Maluf, Hamiltonian Formulation of the Teleparallel Description of the General Relativity. Journal of Mathematical Physical. 35. 335. 1994.

[28] J. W. Maluf, The Gravitacional Energy-Momentum Tensor and the Gravitational Pressure. Annalen der Physik, 14, 723, 2005.

[29] J.W. Maluf, J. F. da Rocha-Neto, Hamiltonian Formulation of General Relativity in the Teleparallel Geometry. Physical Review. D64, 084014, 2001.

[30] J. W.Maluf, F. F. Faria, S. C. Ulhoa, On Reference Frames in Spacetime and Gravitational Energy in Freely Falling Frames. Classical and Quantum Gravity. 24,10, 2007.

[31] F. H. Hehl, J. Lemke e E. W. Mielke, in Geometry and Theoritical Physics, editado por J. Debrus e A. C. Hirshfeld. Springer, Berlim 1991.

[32] S. G. Hahn e R. W. Lindquist, The Two-Body Problem im Geometrodynamics. Annals of Physics. 29, 304, 1964.

[33] M. Shibata e K. Uryu, The Simulation of Merging Binary Neutron Stars in Full General Relativity: Gamma=two Case. Physical Review. D61, 2000.

[34] F. Pretorius, Evolution of Binary Black Hole Spacetimes. Physical Review Letters. 95, 2005.

[35] M. Zilhão e F. Löffler, An Introduction to the Einstein Toolkit. Lecture notes from the NRHEP spring school held at IST-Lisbon, March 2013. 
[36] Cactus Computational Toolkit URL http://www.cactuscode.org/

[37] G. Allen, T. Goodale, G. Lanfermann, T. Radke, D. Rideout e J. Thornburg Cactus Users' Guide 2000 URL http://www.cactuscode.org/Guides/Stable/UsersGuide/ UsersGuideStable.pdf

[38] Carpet: Adaptive Mesh Refinement for the Cactus Framework URL http://www.carpetcode.org/

[39] F. Löffler, F. Joshua, E. Bentivegna, T. Bode, P. Diener, R. Haas, I. Hinder, B. C. Mundim, C. D. Ott, E. Schnetter, G. Allen, M. Campanelli e P. Laguna, The Einstein Toolkit: A Community Computational Infrastructure for Relativistic Astrophysics. Classical and Quantum Gravity. 29, 11, 2012.

[40] The Simulation Factory URL http://simfactory.org/

[41] R. L. Arnowitt, S. Deser e C. W Misner, The dynamics of General Relativity. General Relativity and Gravitation, 40, 1997, 2008.

[42] R. C. Tolman, Static Solutions of Einstein's Field Equations for Spheres of Fluid. Physical Review. 55, 364, 1939.

[43] J. R. Oppenheimer e G. M. Volkoff, On Masive Neutron Cores. Physical Review. 55, $374,1939$.

[44] McLanchlan public BSSN code, URL http://www.cct.lsu.edu/ eschnett/McLachlan

[45] J. D. Brown, P. Diener, O. Sarbach, E. Schnetter e M. Tiglio, Turduckening Blach Holes: An Analytical and Computational Study. Physical Review. D79, 044023. 2009.

[46] C. Reisswig, C. D. Ott, U. Sperhake e E. Schnetter, Gravitational Wave Extraction in Simulations of Rotating Stellar Coe Collapse. Physical Review. D83, 064008, 2011.

[47] Kranc: kranc Assambles Numerical Code, URL http://kranccode.org/

[48] T. W. Baumgarte e S. L. Shapiro, Numereical Intregration of Einstein's Field Equations. Physical Review. D59, 024007, 1998. 
[49] M. Shibata e T. Nakamura, Evolution od Three-dimensional Gravitational Waves: Harmonic Slicing Case. Physical Review. D52, 5428, 1995.

[50] E. Schnetter, Time Step Size Limitation Introduced by the BSSN Gamma Driver. Classical and Quantum Gravity. 27, 16, 2010.

[51] W.Baade e F. Zwicky, On Super-nova. Proceedings of the National Academy of Sciences. 20, 254, 1934.

[52] F. Pacini,Energy Emission from a Neutron Star Nature. 216, 567, 1967.

[53] T. Gold, Rotating Neutron Stars as the Origin of the Pulsating Radio Sources. Nature. 217, 709, 1968.

[54] The official site of the Nobel Prize URL http://www.nobelprize.org/nobel_prizes/physics/ laureates/1993/illpres/discovery.html

[55] K. Y. EKşi. Neutron Stars: Compact Object with Relativisty Gravity. Turkish Journal Physics. 40, 127. 2016.

[56] R. Giacconi, H. Gursky, E. Kellogg, E. Schreier, H. Tananbaum, Discovery of the Periodic X-Ray Pulsations in Centaurus X-3 from UHURU. The Astrophysical Journal. 167, L67, 1971.

[57] R. F. Tooper, The Adiabatic Fluid Spheres in General Relativity. The Astrophysical Journal. 142, 1541, 1965. 